\title{
System identification and control of the standpipe in a cold flow circulating fluidized bed
}

Juchirl Park

West Virginia University

Follow this and additional works at: https://researchrepository.wvu.edu/etd

\section{Recommended Citation}

Park, Juchirl, "System identification and control of the standpipe in a cold flow circulating fluidized bed" (2004). Graduate Theses, Dissertations, and Problem Reports. 2144.

https://researchrepository.wvu.edu/etd/2144

This Dissertation is protected by copyright and/or related rights. It has been brought to you by the The Research Repository @ WVU with permission from the rights-holder(s). You are free to use this Dissertation in any way that is permitted by the copyright and related rights legislation that applies to your use. For other uses you must obtain permission from the rights-holder(s) directly, unless additional rights are indicated by a Creative Commons license in the record and/ or on the work itself. This Dissertation has been accepted for inclusion in WVU Graduate Theses, Dissertations, and Problem Reports collection by an authorized administrator of The Research Repository @ WVU.

For more information, please contact researchrepository@mail.wvu.edu. 


\title{
System Identification and Control of the Standpipe in
}

\section{a Cold Flow Circulating Fluidized Bed}

\author{
by \\ Juchirl Park \\ Dissertation submitted to the \\ College of Engineering and Mineral Resources \\ at West Virginia University \\ in partial fulfillment of the requirements \\ for the degree of \\ Doctor of Philosophy \\ in \\ Electrical Engineering \\ Edward J. Boyle, Ph.D. \\ Muhammad A. Choudhry, Ph.D. \\ Richard Turton, Ph.D. \\ Matthew C. Valenti, Ph.D. \\ Parviz Famouri, Ph.D., Chair \\ Lane Department of Computer Science and Electrical Engineering \\ Morgantown, West Virginia \\ 2004
}

Keywords: System identification, Circulating fluidized bed, Sliding mode, Kalman filter

Copyright 2004 Juchirl Park 


\title{
Abstract \\ System Identification and Control of the Standpipe in a Cold Flow Circulating Fluidized Bed
}

\author{
by \\ Juchirl Park \\ Doctor of Philosophy in Electrical Engineering \\ West Virginia University \\ Parviz Famouri, Ph.D., Chair
}

Circulating fluidized beds (CFB) have been widely applied to many areas of industry such as chemical processing, petroleum refining, catalytic cracker processing, power generation, and waste treatment. The application of CFBs provides several advantages, such as improving gas-solid contact, reducing the cross-sectional area because of high superficial velocities, and having a higher solids flux through the reactor.

A cold flow circulating fluidized bed (CFCFB) has been built at the National Energy Technology Laboratory (NETL), in Morgantown, WV. Recently, a mathematical model of the CFCFB standpipe was successfully developed and tested using an extended Kalman filter $(\mathrm{EKF})$ and an $\mathcal{H}_{\infty}$ estimator algorithm. These estimators, i.e. the $\mathcal{H}_{\infty}$ estimator and EKF algorithms, have been used to estimate states such as the void fraction and the bed-height of the standpipe using pressure drop measurements. Using this standpipe mathematical model requires a solids circulation rate (SCR) to be a measurable variable offered from a spiral installed in the standpipe of the CFCFB. The spiral device at the standpipe of the NETL CFCFB test bed is impractical when a CFB system is operating in a hot temperature unit. In this research, a linear state space system model is developed in order to estimate the solids circulation rate, using a least squares estimator and a subspace algorithm. In this model, the measured aeration flow rates and pressure drops are used as inputs and outputs in the form of a time series in order to build the state space dynamic model. After this model has been obtained, the Kalman filter algorithm is applied in order to estimate the solids circulation rate with the measured pressure drop profiles.

A 2-region model is introduced and discussed as a means of calculating the bed-height using three pressure drop data sets in the standpipe; based on simple pressure drops and void fraction relationships. The standpipe can be modeled as two homogeneous regions, i.e. lean and dense regions. The separation of these two regions is defined as the bed-height.

In this research, a sliding mode estimator is applied in order to estimate the states and the bed-height using a mathematical model with the estimated SCR from the pressure drop profiles. The sliding mode estimator uses the Lyapunov stability criteria to obtain a gain that drives the estimator dynamic to a defined sliding surface, which usually is the first or 
second order differential equation of the error dynamic defined as the difference between the mathematical model equation and the estimator dynamic. Practically, however, the gain can be found from trial and error methods performing several actual simulations. Since the SCR can be estimated from the pressure drop profiles, the sliding mode estimator will need only the pressure drop profiles and the aeration flow rates in order to estimate the state and the bed-height.

Although an entire CFCFB dynamic system has not been built, extensive experimental data sets are available, so a neural network is a strong candidate in building the entire CFCFB system dynamic model. In this research, the neural network is used to obtain the entire system model of the CFCFB for simulation purposes. In the neural network, back-propagation algorithms are adapted and tansig functions are used for the neurons. Since there are linear relationships between the control input (move air) and the regulated outputs i.e. the bed-height and the solids circulation rate, the linear controller is applied in order to control both outputs. The linear controller generates command inputs based on the estimated bed-height and SCR. 


\section{Acknowledgements}

The author would like to express his thanks and gratefulness to his wife, Sunah Park and daughter, Kayla Lee Park for being always beside him with support and encouragement. The author could not have accomplished this research without them. The author also expresses his gratitude to his father and mother for their economic and emotional support. They have always trusted and given the author unquestioning support.

The author also expresses his thanks to his advisor, Dr. Parviz Famouri. Dr. Famouri enabled the author finish this work by hiring him as a GRA and guiding him on the right track. The members of the author's dissertation committee also deserve recognition and thanks from the author for their advice and contributions.

Moreover, the author would like to state that this research has been conducted under a Department of Energy Grant. In addition to funding, the people at NETL have the author's warmest thanks for their support, teaching, answers to various questions that arose in the course of the research, and experimental data.

Finally, the author wishes to acknowledge his friends, Dulpichet Rerkpreedapong, Pisut Rapisak and Hoowang Shim. They have been with the author during his work. The author received much assistance from them in emotional and personal ways. 


\section{Contents}

Acknowledgements iv

List of Figures $\quad$ vii

List of Tables $\quad$ ix

Nomenclature $\quad x$

I Introduction $\quad 1$

1.1 Circulating fluidized bed . . . . . . . . . . . . . . . . . . . . . . . . . . . .

1.1 .1 Combustion application of CFB . . . . . . . . . . . . . . 1

1.1.2 Petroleum refining application of CFB . . . . . . . . . . 2

1.2 Cold flow circulating fluidized bed . . . . . . . . . . . . . . . . . . 6

1.2.1 Overall system of the CFCFB . . . . . . . . . . . . . . . . 6

1.2.2 Advanced instruments in the standpipe . . . . . . . . . . . 8

1.2 .3 Operation of the CFCFB . . . . . . . . . . . . . . . . 9

1.3 Previous research work . . . . . . . . . . . . . . . . . . . . 10

1.4 Current research foci $\ldots \ldots \ldots$. . . . . . . . . . . . . . . . . . . . 13

1.5 Main contributions of the dissertation . . . . . . . . . . . . 16

II One dimensional dynamic model of the standpipe 18

2.1 Introduction . . . . . . . . . . . . . . . . . . . . . . . . . . . . . . . . . . . . .

2.2 Standpipe mathematical model . . . . . . . . . . . . . . . . . . . . . 19

2.2.1 Conservation of the mass and gas flows . . . . . . . . . . . . . . . 19

2.2 .2 State space model of the standpipe . . . . . . . . . . . . . . . . 21

2.3 Standpipe measurement model . . . . . . . . . . . . . . . . . . . . 25

III SCR and bed-height estimates $\quad 28$

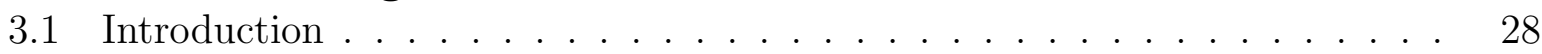

3.2 Preliminaries . . . . . . . . . . . . . . . . . . . . . . . 30

3.2.1 Basic theory . . . . . . . . . . . . . . . . . . 30 
3.2 .2 Least squares approximation . . . . . . . . . . . . . . . . 32

3.3 SCR system development . . . . . . . . . . . . . . . . . . . . . . . . . . 34

3.3 .1 Subspace algorithm . . . . . . . . . . . . . . . . . . . . . . 34

3.3 .2 SCR estimator development . . . . . . . . . . . . . . . . . . . . . . . . . . . . 37

3.4 Kalman filter algorithm . . . . . . . . . . . . . . . . . . . . . . . . . . . . . . 39

3.4 .1 Derivation of a discrete linear Kalman filter . . . . . . . . . . . . . 40

3.4.2 Summary of Kalman filter . . . . . . . . . . . . . . . . . . . . . 42

3.5 Experimental results . . . . . . . . . . . . . . . . . . . . . 43

3.6 Bed-height calculation . . . . . . . . . . . . . . . . . . . . 46

3.6.1 Calculating bed-heights of less than 8.2 meters . . . . . . . . . . . . 49

3.6.2 Modified bed-height calculation method . . . . . . . . . . . 51

3.6.3 Test results . . . . . . . . . . . . . . . . . . . . 52

3.7 Summary . . . . . . . . . . . . . . . . . . . . . . 53

IV Sliding mode estimation technique $\quad 55$

4.1 Introduction . . . . . . . . . . . . . . . . . . . . . . . . . 55

4.2 Variable structure system . . . . . . . . . . . . . . . . . . . . 56

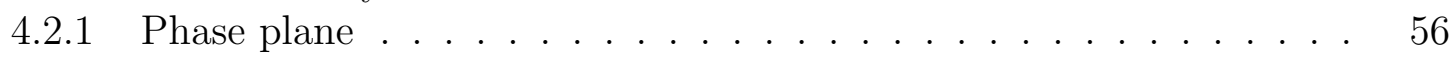

$4.2 .2 \quad$ Basic concept of the sliding mode control . . . . . . . . . . . . . . . 59

4.2.3 Example of a sliding mode control . . . . . . . . . . . . . . . . 63

4.3 Sliding mode estimator for the standpipe of the CFCFB . . . . . . . . 65

4.3.1 Finding the gain based on the Lyapunov stability criterion . . . . . 69

4.4 Simulation results of the CFCFB . . . . . . . . . . . . . . . . . . . 70

4.5 Summary . . . . . . . . . . . . . . . . . . . . . . . 73

V Design linear controller for the SCR and bed-height $\quad 76$

5.1 Introduction . . . . . . . . . . . . . . . . . 76

5.2 Control variable and outputs . . . . . . . . . . . . . . . . . . . . . . . . . . . . . . . . . . . .

5.3 Neural network methods . . . . . . . . . . . . . . . . . . . . . . . . 79

5.3.1 Neural network application to the CFCFB . . . . . . . . . . . . 80

5.3 .2 Results of neural network . . . . . . . . . . . . . . . 82

5.4 Linear controller design . . . . . . . . . . . . . . . . . . . . . . . . . . 84

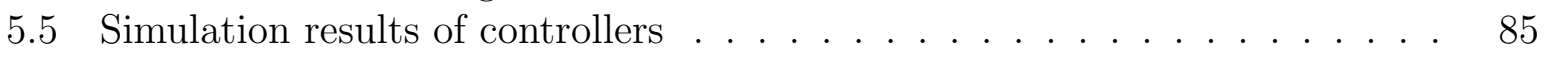

5.6 Summary . . . . . . . . . . . . . . . . . . . 86

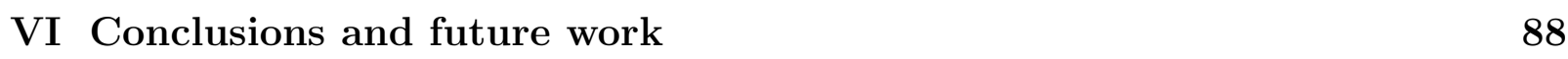

6.1 Conclusions . . . . . . . . . . . . . . . . . . . . . . . 88

6.2 Future work . . . . . . . . . . . . . . . . . . . . 90 


\section{List of Figures}

1.1 Typical diagram of CFB combustion ... . . . . . . . . . . . 3

1.2 Typical flow sheet for the circulating fluid bed boiler system. . . . . . . . . 4

1.3 Schematic diagram of the catalytic cracking application of the CFB. . . . . 5

1.4 Diagram of the CFCFB at the NETL . . . . . . . . . . . 7

1.5 Fiberglass spiral and top view of typical installation. . . . . . . . . . . 9

2.1 Control volume and its definitions . . . . . . . . . . . . . . . . 20

2.2 Detailed propagation diagram of the system model in space and time . . . 25

2.3 Measurement diagram of the standpipe . . . . . . . . . . . . 26

3.1 Discrete Kalman filter timing diagram. . . . . . . . . . . . . . . . . . . 43

3.2 Flow chart of the Kalman filter with linear system . . . . . . . . . . . . . 44

3.3 Experimental results for the SCR (high rate) . . . . . . . . . . . . . 45

3.4 Experimental results for the SCR (low rate) . . . . . . . . . . . . 45

3.5 Experimental results for the SCR . . . . . . . . . . . . . . . 46

3.6 Experimental results for the SCR . . . . . . . . . . . . . . . . . . . 47

3.7 Experimental results for the SCR . . . . . . . . . . . . . . . . 47

3.8 Sample plot of pressure profile vs. heights. . . . . . . . . . . . . . . 49

3.9 Simple homogeneous 2-region model of the standpipe. . . . . . . . . . . 50

3.10 Diagram for the modified 2-region model. . . . . . . . . . . . . . . 52

3.11 Experimental results for the 2-region model . . . . . . . . . . . . . 53

3.12 Experimental results for the modified 2-region model . . . . . . . . . . 54

4.1 Stable node of the phase plane. . . . . . . . . . . . . . . 57

4.2 Unstable node of the phase plane. . . . . . . . . . . . . . . . 57

4.3 Saddle point of the phase plane. . . . . . . . . . . . . . . . . 58

4.4 Center point of the phase plane. . . . . . . . . . . . . . . . . 58

4.5 Stable focus of the phase plane. . . . . . . . . . . . . . . . . . 59

4.6 Unstable focus of the phase plane. . . . . . . . . . . . . . . . . . 60

4.7 Second order system for sliding mode controls . . . . . . . . . . . . . 60

4.8 Basic concept of a sliding mode control. . . . . . . . . . . . . . . . 61 


\section{LIST OF FIGURES}

4.9 Basic concept of a sliding mode control using a switching line. . . . . . . . 62

4.10 Simulation results of a sliding example . . . . . . . . . . . . . . . . 64

4.11 Simulation results of a phase plot of a sliding mode example . . . . . . . 65

4.12 Simulation results of a sliding example with the unstable system . . . . . . 66

4.13 Simulation results of a sliding example with unstable system . . . . . . . . 66

4.14 Pressure profile calculation for the $\Delta p^{2} \ldots \ldots \ldots \ldots$. . . . . . . . . 67

4.15 Flow chart of the sliding mode estimator. . . . . . . . . . . . . . . . . . . . 68

4.16 Detail representation of the standpipe . . . . . . . . . . . . . . . . 71

4.17 Simulation results of a sliding mode estimator . . . . . . . . . . . . . 73

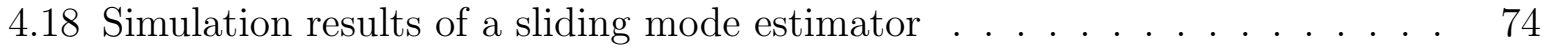

4.19 Simulation results of a sliding mode estimator . . . . . . . . . . . . . . 74

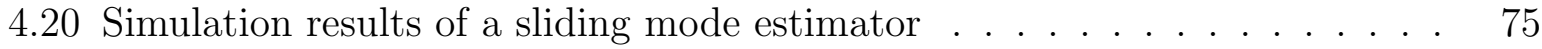

5.1 Relationship between move air and the SCR. . . . . . . . . . . . . . 77

5.2 Relationship between move air and the SCR. . . . . . . . . . . . . 78

5.3 Relationship between move air and bed-height. . . . . . . . . . . . . . . 78

5.4 Relationship between move air and bed-height. . . . . . . . . . . . . . . . . 79

5.5 Detailed diagram of training neural network . . . . . . . . . . . . . . . 82

5.6 The SCR measured and synthesized from neural network. . . . . . . . . . . 83

5.7 The SCR measured and synthesized from neural network. . . . . . . . . . . 83

5.8 Block diagrams for the linear and system models with a neural network. . . 84

5.9 Simulation results for the controller . . . . . . . . . . . . . . 86

5.10 Simulation results for the controller . . . . . . . . . . . . . . . 87 


\section{List of Tables}

1.1 Characteristics of Corks . . . . . . . . . . . . . . . . 10

3.1 Detailed input and output components. . . . . . . . . . . . . . . 38

3.2 Operating conditions of the CFCFB for building the SCR system model. . $\quad 39$

3.3 Operating conditions of the CFCFB to test the SCR system model. . . . . 44

4.1 Output and input tags and their components of the sliding mode estimator. 72

5.1 Definitions for neural network training with inputs and outputs. . . . . . . 81 


\section{Nomenclature}

$\varepsilon \quad$ Void fraction of the gas, page 20

$\varepsilon_{m f} \quad$ Void fraction at minimum fluidization, page 22

$\varepsilon_{p b} \quad$ Void fraction at packed bed, page 22

$\mu \quad$ Fluid viscosity, page 26

$\nabla \quad$ Divergence operator, page 19

$\rho_{g} \quad$ Gas density, page 26

$\rho_{p} \quad$ Particle density, constant, page 20

$\rho_{s} \quad$ Aggregate density, variant, page 20

$\rho_{s}(\vec{r}, t)$ Density of the solid with respect to time and space, page 18

$\overrightarrow{\jmath_{g}} \quad$ Volumetric flux of the gas, page 20

$\overrightarrow{\jmath_{s}} \quad$ Volumetric flux of the solid, page 20

$\vec{\jmath}_{T} \quad$ Total volumetric flux, page 20

$\vec{n} \quad$ Normal vector to the surface, page 18

$\vec{v}_{r} \quad$ Relative speed, page 21 


\section{Nomenclature}

$\vec{w} \quad$ Velocity vector to the surface, page 18

$C_{1}, C_{2}$ Some constant, page 26

d Effective particle diameter, page 26

$M(t)$ Total mass of a given volume, page 18

$N_{p} \quad$ Number of pressure drop profiles, page 26

$N_{s p} \quad$ Number of discretized cells, page 26

$V_{t} \quad$ Terminal velocity of the isolated particle, page 22

$\bar{A}, \bar{B} \quad$ Arbitrary matrices for SCR dynamics, page 36

$\Delta P_{1} \quad$ Overall pressure drop in lean phase, page 48

$\Delta P_{2} \quad$ Overall pressure drop in dense phase, page 48

$\Delta P_{T} \quad$ Total pressure drop in the standpipe, page 48

$\hat{x}_{k}(+)$ Posterior estimated vector, page 39

$\hat{x}_{k}(-)$ Prior estimated vector, page 39

U Input formed matrix from the input vector, page 34

X State formed matrix from the state vector, page 34

Y Output formed matrix from the output vector, page 34

$\mu \quad$ Mean value of time series, $z_{t}$, page 29

$\mu(t) \quad$ Noise vector, page 32

$\Phi_{k-1}$ Discrete time variant state matrix, page 38 


\section{Nomenclature}

$\psi(t) \quad$ Previously known input and/or output vector, page 32

$\theta \quad$ Regression vector, page 32

$A, B$, and $C$ Matrices for the state space dynamic model, page 33

$a_{t} \quad$ Noise term of the time series, page 30

$E[\cdot] \quad$ Expected value of an arbitrary function, page 29

G Input output data formed matrix, page 35

$g \quad$ The gravitational acceleration, page 46

H Output matrix contains only pressure profiles, page 37

$H_{k} \quad$ Discrete time variant measurement matrix, page 38

$K_{k}^{\prime} \quad$ Constant matrix for recursive filter, page 39

$K_{k} \quad$ Constant gain matrix for a discrete linear Kalman filter, page 39

$N \quad$ Number of the elements, page 34

$n \quad$ Dimension of matrix $A$, page 33

$n \quad$ Dimension of the system state vector, page 36

$N_{p} \quad$ Dimension of the output vector, page 36

$N_{t} \quad$ Total number element of the time series, page 29

$O_{r} \quad$ Extended observability matrix, page 33

$p(z) \quad$ Probability function of the time series $z_{t}$, page 29

$P_{k}(+)$ Posterior error covariance matrix, page 40 


\section{Nomenclature}

$Q, R$ Covariance matrices for the discrete Kalman filter, page 39

$R \quad$ Full rank matrix, page 35

$S, S_{1}$ Some matrices found from the SVD, page 35

$S_{r} \quad$ Arbitrary matrix, page 34

$T \quad$ Arbitrary invertible matrix, page 33

$U_{r}(t)$ Input vector form from input data, page 34

$v(t) \quad$ Measurement noise vector, page 33

$w(t) \quad$ State noise vector, page 33

$W_{1}$ and $W_{2}$ Some weighted matrices for subspace algorithm, page 35

$x_{f}(t)$ Fictitious state for SCR dynamics, page 36

$y(t) \quad$ Output state vector with only the pressure profiles, page 37

$Y_{r}(t)$ Output vector form from output data, page 34

$z_{t} \quad$ Time series, page 29

$\alpha, \beta \quad$ Some constant, page 55

$\bar{\varepsilon} \quad$ Amount contribute to each element of the state vector, page 68

$\bar{\Lambda} \quad$ Error correction from the sliding mode observer, page 68

$\dot{x} \quad$ Time derivative of the state, page 55

$\hat{\imath} \quad$ Estimated variables, page 66

$\Lambda^{j} \quad j^{\text {th }}$ switching line for the sliding mode estimator, page 66 


\section{Nomenclature}

M Matrix convert pressure drops to state components, page 68

a Constant real number, page 59

$a_{1}, a_{2}$ Some constant, page 55

$e^{j}(t) \quad j^{t h}$ pressure error at time, $t$, page 66

j Imaginary number, i.e. $\sqrt{-1}$, page 55

$K^{j} \quad j^{\text {th }}$ gain matrix for the sliding mode estimator, page 65

$p^{j} \quad$ Pressure measurement in $j^{\text {th }}$ cell, page 65

$V(\vec{x}, t)$ Arbitrary positive energy function,, page 54

$z_{\text {act }}$ Actual positions of the pressure measurements, page 65

$\bar{T}(k) \quad$ Average value of output, page 77

$\bar{u}(k) \quad$ Average value of input, page 77

$\gamma_{1}, \gamma_{2}, \gamma_{3}, \gamma_{4}$ Arbitrary constants, page 74

$\mathbf{H}_{n} \quad$ Hessian matrix in back propagation training, page 77

$\mathbf{J}_{n} \quad$ Jacobian matrix in back propagation training, page 77

$\mu_{n} \quad$ Constant, page 77

$x_{n}(k)$ Neuron state function, page 77 


\section{Chapter I}

\section{Introduction}

\subsection{Circulating Fluidized Bed}

In a variety of industrial applications, the use of a circulating fluidized bed (CFB) provides various advantages, such as reducing environmental pollution and increasing process efficiency. The application of circulating fluidized bed technology contributes to the improvement of gas-solid contact, reduction of the cross-sectional area with the use of higher superficial velocities, the use of the solids circulation rate as an additional control variable, and superior radial mixing, Grace et al. [1]. Since CFBs have industrial applications such as power generation, Basu and Fraser [2], chemical processing, petroleum refining, and catalytic cracker processing, King [3], applications in combustion and refining processes are described and discussed in this section.

\subsubsection{Combustion Application of CFB}

The first example discussed in this section is a combustion application that has a single solids circulation loop and a single gas feed. These types of units have a single standpipe for the solids and a single reaction vessel. A circulating bed coal combustor in which 
limestone and injected coal are circulated by air is the common example of this type of CFB application. A typical schematic diagram of a circulating fluidized bed combustion (CFBC) system is shown in Fig. 1.1, Kullendorff and Andersson [4]. It consists of a furnace region, a recycle region, a cyclone, and a heat transfer unit. An aeration injection point can be located in either of two places, i.e. the bottom and middle points of the furnace region. However, the configuration possibilities for the CFBC depend on the fluidization velocity, primary to secondary air ratio, bed densities, and different heat transfer locations, Kullendorff and Andersson [4]. In Fig 1.1, the heat transfer surfaces are located in and around the furnace bed. On the other hand, a compact external heat exchange boiler greatly enhances both the fuel flexibility and load control capability of CFB boilers, Wang et al. [5]. The schematic diagram of the CFBC for the external heat exchange is shown in Fig. 1.2, Hirsch et al. [6]. In this diagram, the heat transfer surfaces are located in a convective pass after the cyclone. Regardless of the configuration of the CFBC, this system burns an air-mixed fuel, fed into the riser to improve the combustion efficiency and reduce pollutants such as $\mathrm{SO}_{x}$ and $\mathrm{NO}_{x}$. Furthermore, a limestone feed is required for fuels with sulfur content greater than $0.5 \%$, Kavidass et al. [7]. The primary air flow lifts and mixes the solids with aeration air, so the solids (i.e. the fuel) are burned efficiently and produce less residues. The unburned solids pass through the cyclone to separate and vent the air from the solids. These solids are recycled and fed back to the riser (i.e. the furnace bed). The heat is conveyed from the CFBC to make steam, which is sent to a turbine in applications involving electric power generation. Since the fuel is well mixed by aeration air, the burning processes are more efficient than they would be in a conventional boiler.

\subsubsection{Petroleum Refining Application of CFB}

In this section, another typical application of the CFB, namely fluidized catalytic cracking (FCC) is presented and discussed. In fact, the earliest application of the circulating 


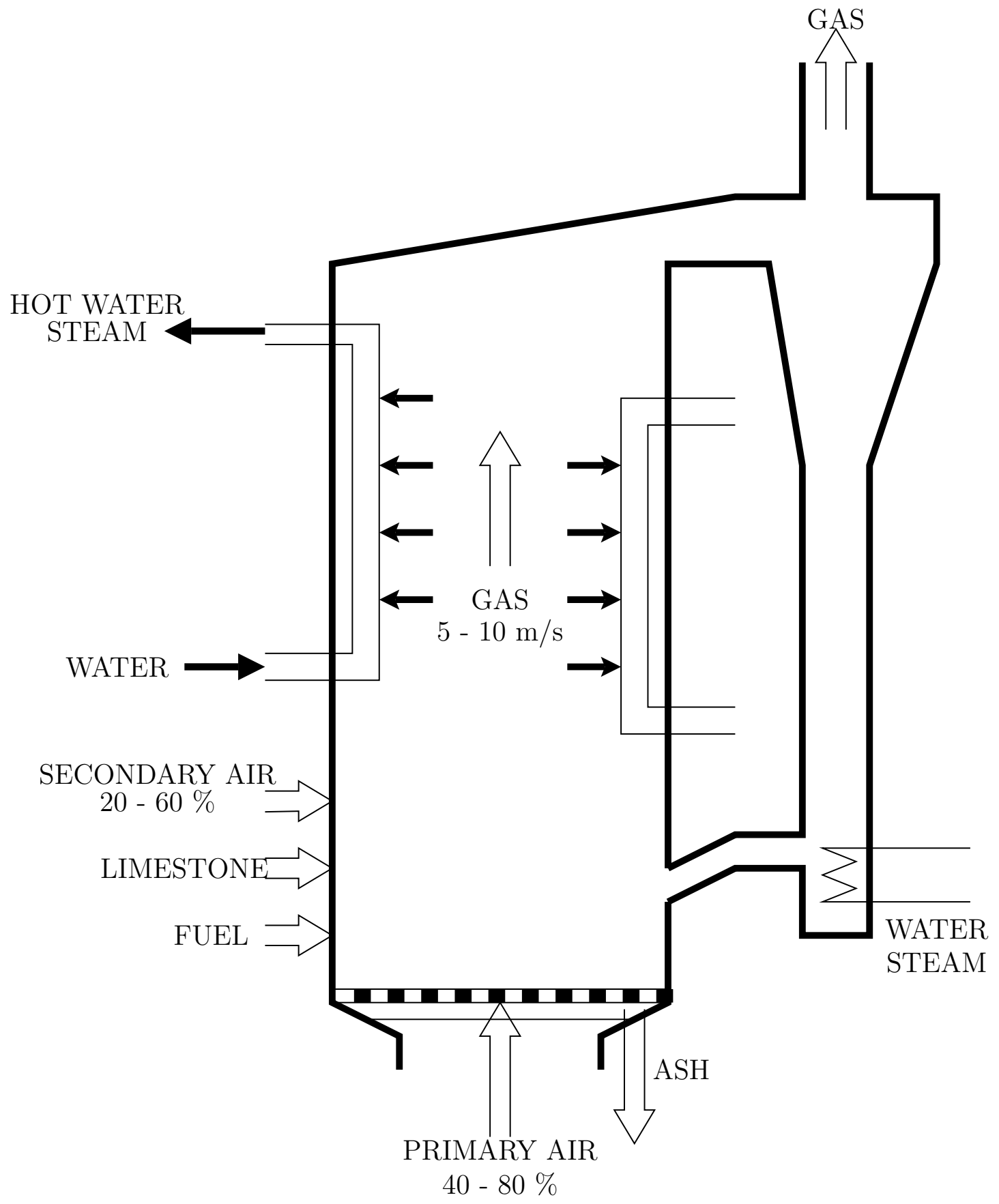

Figure 1.1: Typical circulating fluidized bed combustion application schematic, Kullendorff and Andersson [4]. 


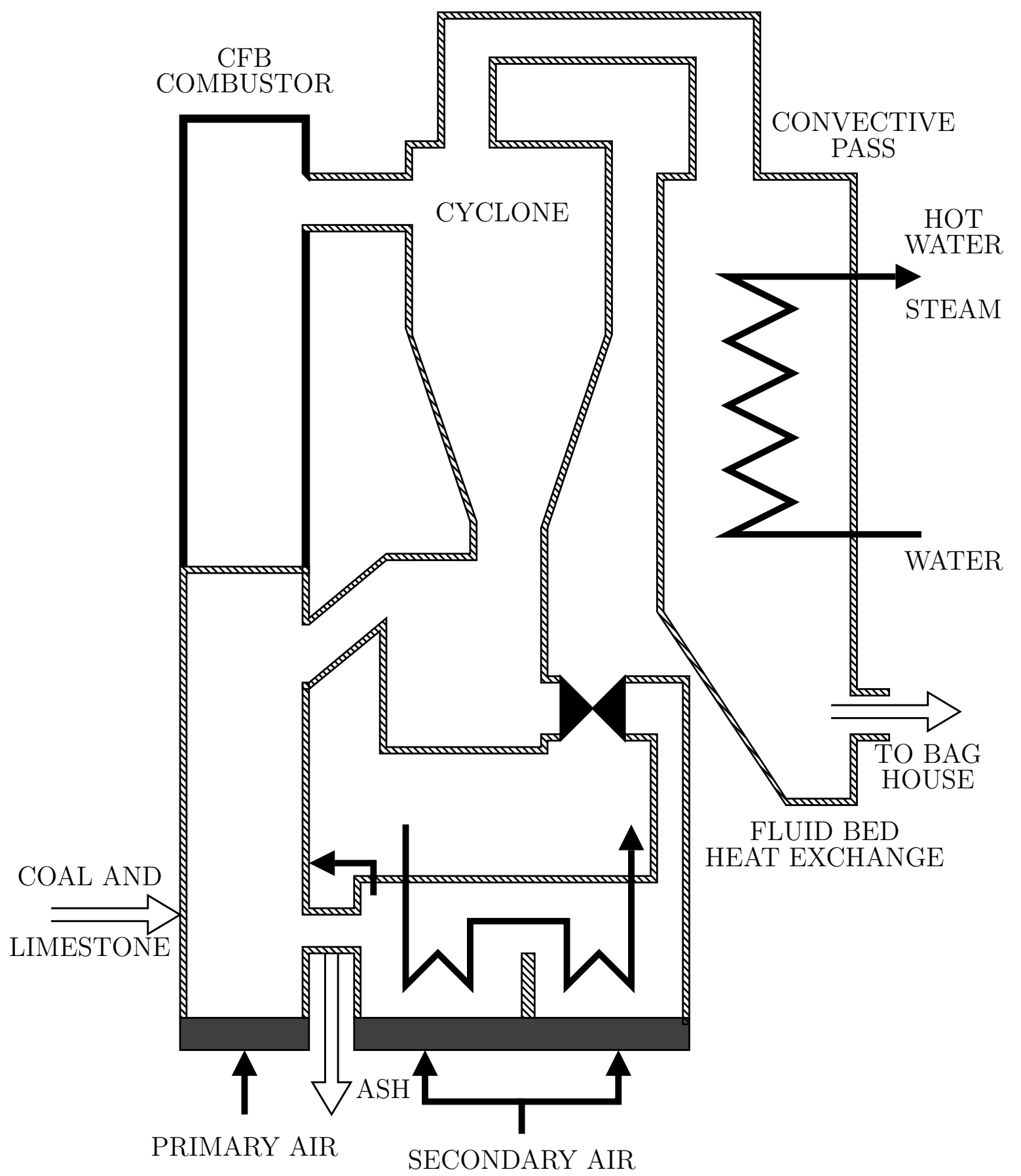

Figure 1.2: Typical flow sheet for the circulating fluid bed boiler system, Hirsch et al. [6]. 
fluidized bed was the catalytic cracking process, developed after Eugene Houdry discovered that a porous clay catalyzed the cracking reaction of gas/oil to produce high octane gasoline at atmospheric pressure, Squires [8]. A typical diagram of the catalytic cracking process is shown in Fig. 1.3, SET Laboratories Inc. [9]. In common fluid catalytic cracking (FCC)

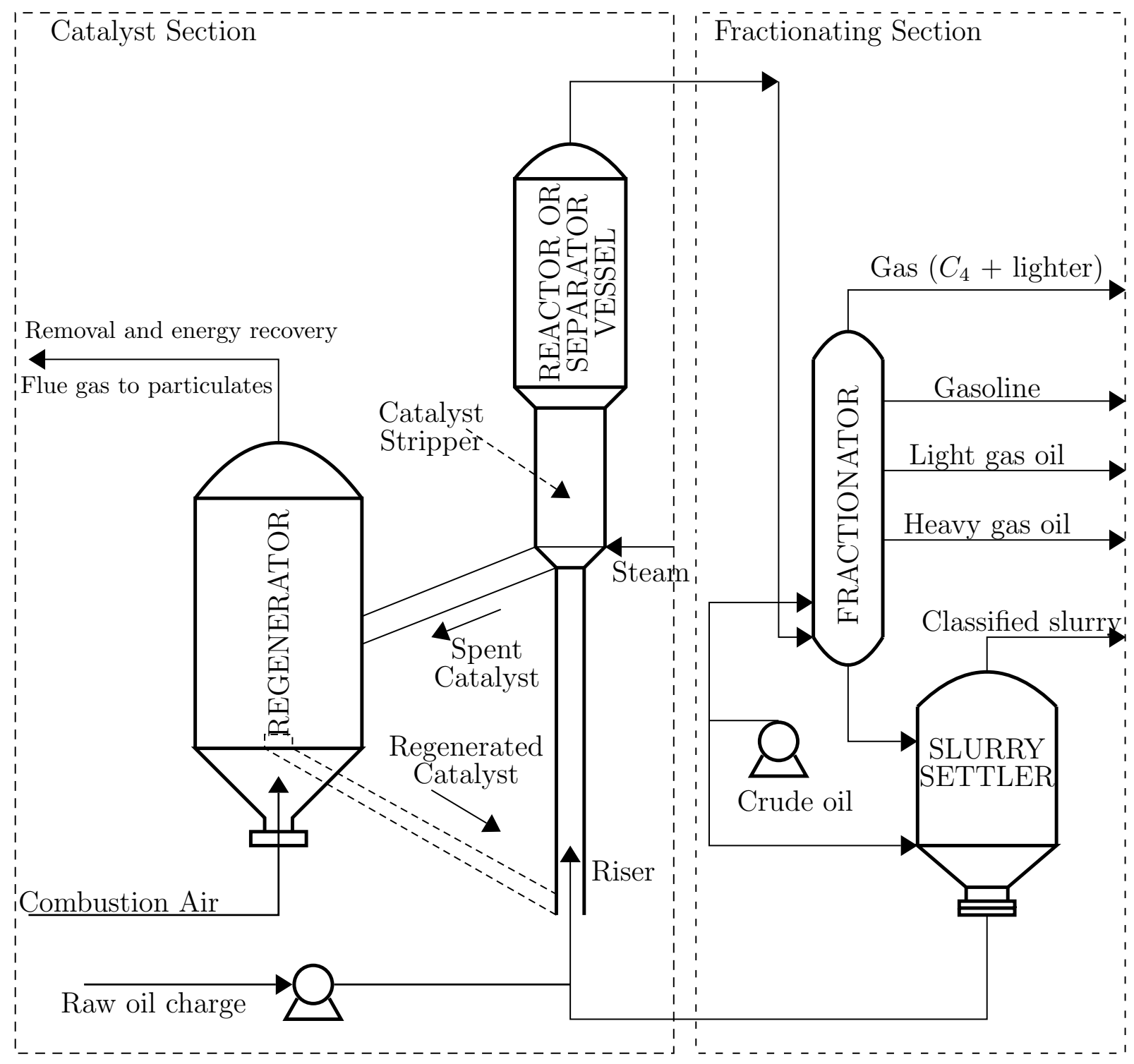

Figure 1.3: Schematic diagram of the catalytic cracking application of the CFB, SET Laboratories Inc. [9].

processes, there are two sections, the catalyst and fractionating sections. The fractionating 
section consists of a fractionator and slurry settler, while the catalyst section contains a riser, a reactor, and a regenerator, SET Laboratories Inc. [9].The typical operation of FCC processes includes a preheated hydrocarbon charge with a hot, regenerated catalyst that enters the riser leading to the reactor, SET Laboratories Inc. [9]. Next, the charge is mixed with a recycled flow within the riser, gasified, and enters the reactor at a temperature of $483{ }^{\circ} \mathrm{C}-538{ }^{\circ} \mathrm{C}$. The charge is cracked in the riser under a pressure around of $68950-$ 206850 PASCAL. The cracking continues until the cyclone separates the vaporized oil from catalyst. Next, the cracked product is sent to a fractionator and is divided into several fractions. The heavy oil is recycled and fed back to the riser. In the regenerator block, preheated air regenerates and mixes the spent catalyst. For optimization of the cracking process, the fresh catalyst is refilled while the worn-out catalyst is removed, SET Laboratories Inc. [9]. In more modern FCC processes, however, all catalytic processing happens in the riser.

\subsection{Cold Flow Circulating Fluidized Bed}

\subsubsection{Overall System of the CFCFB}

The current research project is based on experimental data collected from a cold flow circulating fluidized bed (CFCFB) test facility installed at the National Energy Technology Laboratory (NETL), in Morgantown, West Virginia. This CFCFB test facility was built at NETL during the 1960s. The system was built for studying the behavior and operation of circulating fluidized bed systems under cold conditions. However, in industrial settings most CFBs operate at high temperatures, Koduru [10]. A simplified diagram of the CFCFB built at NETL, in Morgantown, West Virginia is shown in Fig. 1.4, with indicated dimensions, Park et al. [11, 12]. The $15.45 \mathrm{~m}$ high CFCFB test facility consists of a standpipe, a non-mechanical valve, a riser, a gas/solid separator, and other advanced 


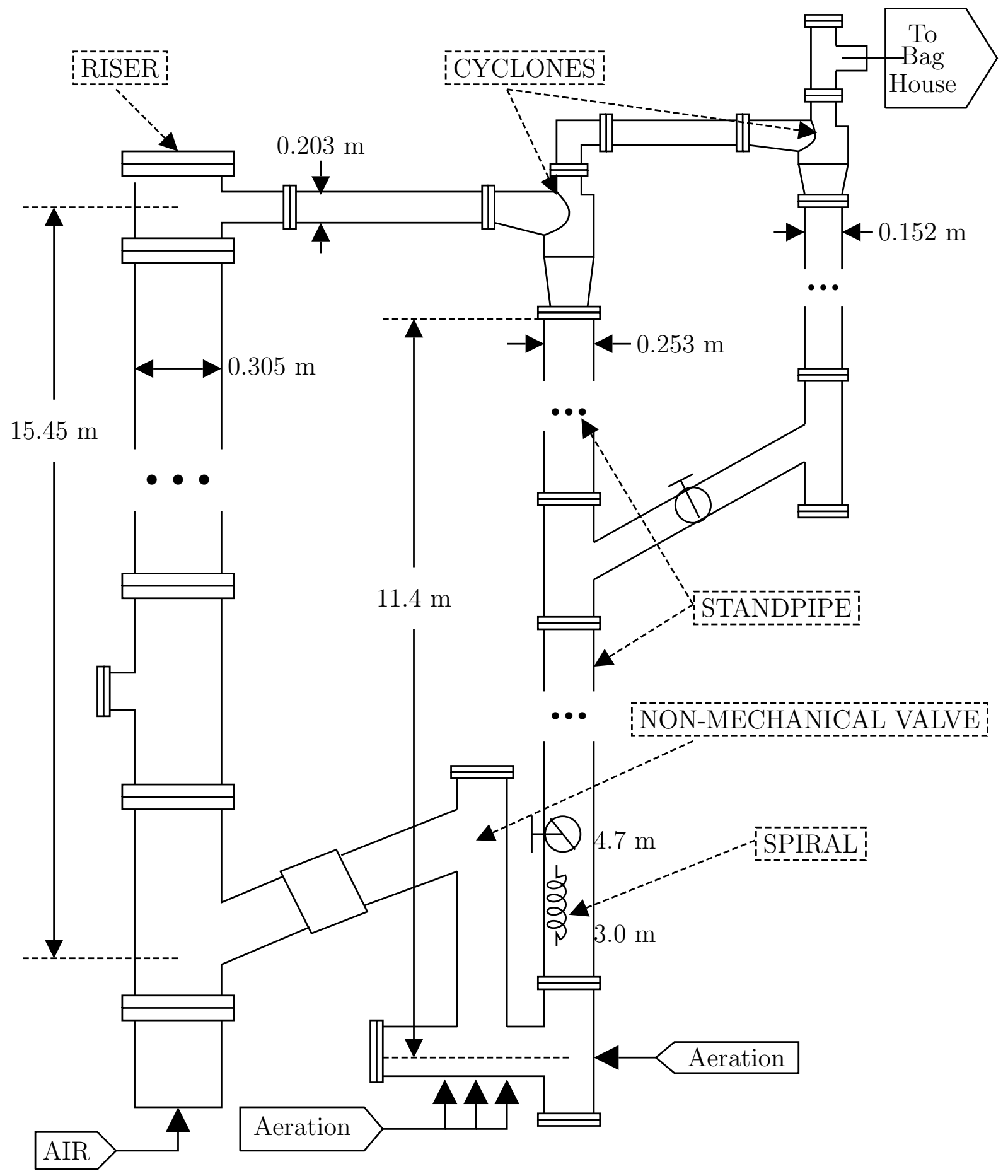

Figure 1.4: The simplified diagram for the NETL cold flow circulating fluidized bed test facility, Park et al. [11, 12]. 
measuring instruments, such as a spiral for measuring the solids circulation rate, a density meter for bulk density, etc. The standpipe is $11.40 \mathrm{~m}$ high and $0.253 \mathrm{~m}$ in diameter, as indicated in Fig. 1.4. It has a section of clear acrylic to allow interior visibility, eight taps to measure the pressure drop, a spiral, and four aeration flow injection points to maintain fluidization. The non-mechanical valve is called an L-valve or a Z-valve because of its shape. It is $1.5 \mathrm{~m}$ high, and $0.253 \mathrm{~m}$ in diameter in its standing portion and $0.227 \mathrm{~m}$ in diameter in its slanted portion. The valve has air injection points at the bottom to maintain fluidization of the solid material. The riser is $15.45 \mathrm{~m}$ high and $0.305 \mathrm{~m}$ in diameter and is built of metal, so the contents cannot be seen from the outside. The riser also has many pressure taps to facilitate accurate pressure drop measurement. The riser is connected to a gas/solid separator (i.e. a cyclone) at the top of the standpipe. This cyclone connects to another cyclone in order to separate the gas from the solids and to discharge the gas from the system. The separated solids are returned to the standpipe to create a dense bed.

\subsubsection{Advanced Instruments in the Standpipe}

A twisted fiberglass vane solids flow meter installed in the standpipe of the CFCFB allows measurement of the solids circulation rate. The fiberglass spiral and typical installation in the standpipe are shown in Fig. 1.5, Ludlow et al. [13]. The meter has two parts, the twisted vane and rotation electronics, to detect the rotation of the vane as shown in Fig. 1.5-a. The twisted vane, or spiral, is shaped and twisted to be rotated by the solids flow. The typical orientation of the spiral in the standpipe is hanging down along the centerline of the standpipe; with the electronics remaining outside the standpipe as shown in Fig. 1.5-b. The spiral is installed in the packed bed of the standpipe to allow rotation by the motion of the solids as they move down in the standpipe, Ludlow et al. [13]. The twisted vane is used because it allows a continuous flow measurement. The collected data is sampled at $1 \mathrm{~Hz}$. There are several other advanced instruments, such as a Laser Doppler 


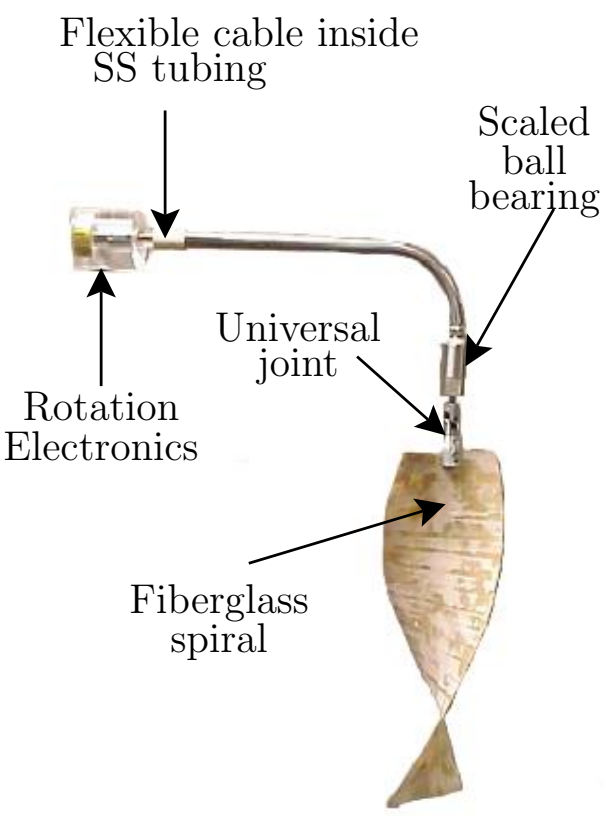

(a) Twisted fiberglass vane

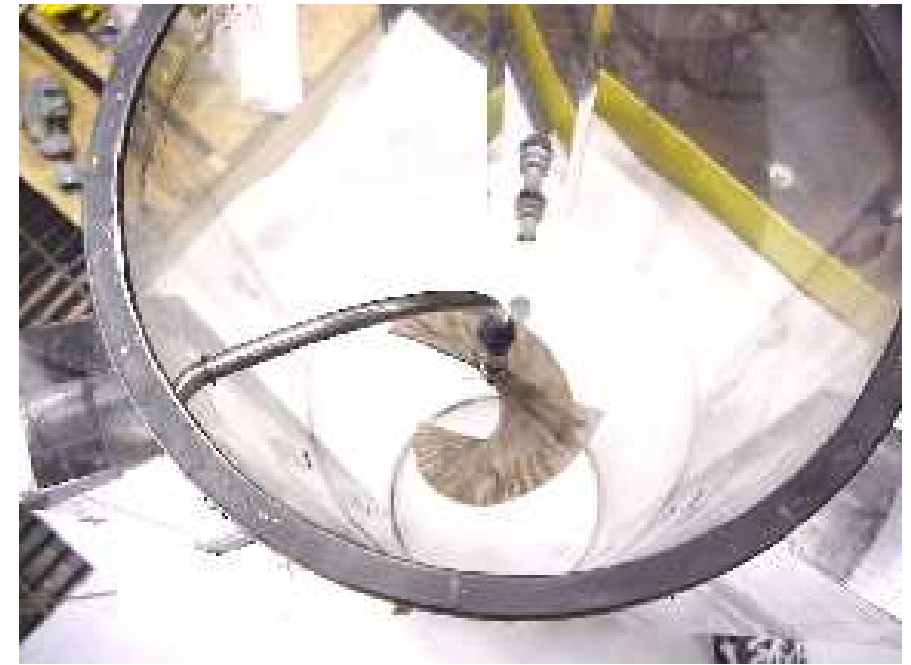

(b) Top view of spiral installation

Figure 1.5: Fiberglass spiral and top view of typical installation.

Velocimeter, which measures particle velocity near the riser wall, and a Solid Sampling Probe, which measures solid flux, etc. These other instruments are not discussed here since they are not related to this research.

\subsubsection{Operation of the CFCFB}

The pressure drop measurements are sampled at a rate of $1 \mathrm{~Hz}$ by a data acquisition system that stores the data for offline processing. The solid material being tested is cork, which has an average diameter of $812 \mu \mathrm{m}$ and is used to represent the coal particles used in high temperature and pressure applications. Detailed bed material characteristics for the cork are shown in Table 1.1 .

First, the mass of solids is aerated with a high air flow at the bottom of the riser. After reaching the top of the riser, the mass is transferred to a two-stage gas/solid cyclone 


\begin{tabular}{l|c|l}
\hline \hline \multicolumn{3}{l}{ Cork characteristics: } \\
\hline Solid particle density (cork) & $\rho_{s}$ & $189 \mathrm{~kg} / \mathrm{m}^{3}$ \\
\hline Bulk density & $\rho_{b}$ & $95 \mathrm{~kg} / \mathrm{m}^{3}$ \\
\hline Effective cork diameter & $d_{v s}$ & $812 \mu \mathrm{m}$ \\
\hline Voidage at minimum fluidization & $\varepsilon_{m f}$ & 0.49 \\
\hline Particle terminal speed & $\mathrm{V}_{t}$ & $0.86 \mathrm{~m} / \mathrm{s}$ \\
\hline Minimum fluidization velocity & $\mathrm{U}_{m f}$ & $0.07 \mathrm{~m} / \mathrm{s}$ \\
\hline \hline
\end{tabular}

Table 1.1: Characteristics of Corks

that separates gas from solids and vents the gas outside. The solids then fall down in the standpipe and accumulate to create the bed level. This free falling region is called the lean phase. The area below the bed level is the region defined as the dense bed, which is a fluidized flow of solids. The air injected at the base of the standpipe is referred to as move air and is one of the primary controls of the system. As the move air is changed, the solids circulation flow changes, causing the bed-height to change. Other air injections occur but are very small and have little effect on the bed-height. In the lean phase region of the standpipe, there is no significant change of pressure, but there is a significant change in the dense bed because of the packed solids. This is where fluidization occurs. The air injected at the bottom of the riser is kept constant, moving the solids through the riser.

\subsection{Previous Research Work}

In the next few paragraphs, previous research on $\mathrm{CFB}$ applications and the CFCFB are discussed, primarily in relation to combustion, catalytic cracking, and waste treatment applications of the CFB. Next, research work related to the CFCFB is explained and discussed. Stoholm et al. [14] present an example of a circulating fluidized bed gasification process for burning biomass and many waste materials at low temperature conditions. This research also demonstrates the use of CFB technology to provide off-gas system compact- 
ness. The use of a CFB boiler in plastic recycling is explained and discussed by Milne et al. [15]. The internally circulating fluidized bed reactor has the capability to convert waste plastics to olefins, Milne et al. [15]. Potential uses of the ash are given in Conn et al. [16] and include soil stabilization, road base, structure fill, and synthetic aggregate. This research work also characterizes the type of ash and explains the various uses of ash. In Tame [17], the principle of a revolving-type fluidized bed incinerator is explained and discussed. This paper also describes technologies such as bed temperature control and bed desulferization for recycling the waste material through the use of a fluidized bed. A coal fired CFB boiler is modeled in a dynamic simulation using hydrodynamics, heat transfer, and combustion in Huilin et al. [18]. This dynamic simulation can predict the chemical gas species and char concentration distributions in both axial and radial directions, Huilin et al. [18].

A survey of the catalytic cracking industry and the application of CFB over the past 50 years can be found in Reichle [19]. This paper shows that CFBs are applied not only for catalytic cracking but also to other chemical reactors. CFBs can be utilized in various processes such as the combustion of coal, wood and shale, the gasification of coal and biomass, the simultaneous removal of $\mathrm{NO}_{x}$ and $\mathrm{SO}_{2}$ from off-gases, fluid catalytic cracking, and dry scrubbing applications, Herrmann and Weisweiler [20]. Desulferization can be achieved with dry scrubbing using $\mathrm{Ca}(\mathrm{OH})_{2}$, while removal of $\mathrm{NO}_{x}$ is performed by selective catalytic reduction with $\mathrm{NH}_{3}$, Herrmann and Weisweiler [20]. An experimental study of the circulating fluidized bed in a catalytic cracking reactor is given in Quyang and $\mathrm{Li}$ [21]. In this paper, the performance of a $\mathrm{CFB}$ with a $0.25 \mathrm{~m}$ diameter riser is given. and the effects of its operating conditions are given. CFB boiler emission controls have been studied by Beacon and Lundqvist [22]. In this research, successful achievement of low levels of $\mathrm{SO}_{2}$ and $\mathrm{NO}_{x}$ in the $50 \mathrm{MW}_{e}$ ASHLESTROM PYROFLOW CFB boiler is also presented.

The following discussion presents numerous research projects relating to cold flow cir- 


\section{CHAPTER I. INTRODUCTION}

culating fluidized beds (CFCFB). The development of a spiral device to measure the solids circulation rate is presented and explained by Ludlow et al. [13]. That paper includes the detailed design, installation, and performance of a twisted vane measuring device in the standpipe. The overall solids circulating rate is calculated using the speed of rotation of the twisted vane, the bulk density of solids, and cross-sectional area of the standpipe, Ludlow et al. [13]. Lawson and Shadle [23] describe a cold flow circulating fluidized bed that uses polyvinyl chloride particles, in which the hydrodynamics on the legs of the CFCFB were characterized. The control strategies for open and close loops are provided. In addition to the control, the process variables and their dependencies are identified and characterized experimentally. Furthermore, determination of the operational regime of the CFCFB was studied by Shadle et al. [24]. Flow instabilities under riser pressure fluctuations and solids circulation rates were studied by Shadle et al. [24] under conditions in which the CFCFB was operating in the intermediate, turbulent, and fast fluidization flow regimes.

Attempts to model and control a CFCFB are given by Koduru [10], Davari et al. [25]. In these papers, neural networks are applied in order to model the CFCFB, using a huge amount of data collected from experiments. In addition to modeling, the control of the solids circulation rate using a neural network was considered by Koduru [10]. Shim et al. [26] developed a standpipe model, using the conservation of solid and gas flows. In addition to a dynamic model, an extended Kalman filter (EKF) algorithm was successfully applied in order to estimate the state and the bed-height in the standpipe using an approximated standpipe state space system model and an experimentally measured solids circulation rate from a spiral installed in the standpipe, Shim et al. [26]. Furthermore, $\mathcal{H}_{\infty}$ estimation methods were successfully applied to some oscillating cases in which the EKF failed, Park et al. [11]. The $\mathcal{H}_{\infty}$ estimation methods use the same algorithms and approximations of the system model as those of the EKF. 


\subsection{Current Research Foci}

The primary research focus of this dissertation is system identification and control of the CFCFB. In addition to the extended Kalman filter and $\mathcal{H}_{\infty}$ techniques, other robust techniques such as the sliding mode estimation is applied in order to estimate the state and the bed-height. There is a large amount of experimental data and information available at the test facility of DOE/NETL. This allows detailed and accurate numerical and systematic methods to be studied and investigated allowing the formulation of a system model to estimate the solids circulation rate. Furthermore, since the clear acrylic portions of the standpipe in the NETL CFCFB test bed allow the bed-height to be observed and recorded, a simple method of calculating the bed-height can be developed. Linear control theory was applied to regulate the solids circulation rate and bed-height because both variables can be measured experimentally. An analytical model for the entire CFCFB is not available. However, because a large quantity of data has been collected, a neural network may be applied in order to build a system model with which to test the linear controller.

The challenges are to develop a standpipe system model, to estimate the state variables, to calculate the bed-height, to estimate solids circulation rate, and to control both bedheight and solids circulation rate using linear control theory. The solids circulation rate is one of the most important parameters in the operation of the CFB, since it affects mass and heat transfer characteristics, which in turn impact the efficiency of the processes. Several techniques have been used to determine this variable, Burkell et al. [27], Lui and Bowen [28]. However, the measurement of this parameter is very difficult in industrial-scale CFB units, operating under extreme process conditions. In this research, however, since the CFCFB operates under cold conditions, the SCR can be measured with an advance instrument, namely the spiral installed in the standpipe of the CFCFB. From these measured values, the linear system model for the solids circulation rate can be built, which is discussed in Chapter III and is used to estimate the SCR with a discrete Kalman filter. The linear 
dynamic model for this purpose is defined as,

$$
\begin{aligned}
x(k+1) & =A x(k)+B u(k), \\
y(k) & =C x(k),
\end{aligned}
$$

where $x(k)$ is the state vector, $y(k)$ is the output vector, and $u(k)$ is the input vector, while $A, B$, and $C$ are constant matrices. The least squares estimator and an extended observability matrix are utilized through a subspace algorithm, Moonen and Ramos [29], Swindlehurst et al. [30] in order to estimate the matrices $A, B$, and $C$ of the state space system model, Ljung [31]. The least squares method is developed to estimate the parameters for the auto regressive moving average (ARMA) or other time series model forms, using the experimental data, Brotherton and Caines [32], Goethals et al. [33], Bai and Nagpal [34]. The system model is then transferred to another form containing only pressure drop data, which are the only available measurements. The discrete Kalman filter is used to estimate the SCR, based on the linear state space dynamic system model with measured pressure drop profiles and aerations.

In addition to the Kalman filter estimation, in this research other robust estimating methods are also studied, such as a sliding mode observer. The sliding mode observer has been applied in estimating the current, voltage, or speed of an induction machine, Yan et al. [35], Utkin [36], Benchaib et al. [37], Derdiyok et al. [38], Rehman et al. [39], Rodic and Jezernik [40]. The system models used in the sliding mode estimator are usually nonlinear and are manipulated according to the needs of the estimator. Yan et al. [35], Utkin [36], Benchaib et al. [37] illustrate the development of a sliding estimator based on the mathematical model of an induction machine, showing the technique's superiority in dealing with tolerances to noise and parameter deviations. However, there are no mathematical or generalized procedures for finding the sliding surface or determining the structure of the sliding mode estimator, based on the system model. In this research, since the SCR is available from the estimator, a sliding mode observer uses the estimated SCR in order to 


\section{CHAPTER I. INTRODUCTION}

estimate the state and the bed-height of the standpipe, using the previously developed mathematical standpipe model.

Furthermore, the developed algorithm integrates a 2-region model that uses 3-pressure measurements from the standpipe to calculate accurately the bed-height, using the basic pressure and void fraction relationship defined as,

$$
\Delta p=\rho_{s}(1-\varepsilon) g h,
$$

where $\Delta p$ is the pressure drop, $\rho_{s}$ is the density of solid, $\varepsilon$ is the void fraction, $g$ is the gravitational acceleration, and $h$ is the length over which the pressure drop is measured.

The SCR is successfully estimated and the bed-height is accurately calculated, using methods developed in this research. The control of these quantities is also a research topic of this dissertation. Linear system control theory is mature, well understood, and commonly used in industry applications. A linear controller is developed here to control the SCR and the bed-height, in order to improve the reliability and robustness of the system. For simulation purposes of testing the linear controller, an entire mathematical model of the CFCFB system is required. On the other hand, a tremendous quantity of experimental data is available, allowing the entire CFCFB system model to be built using a neural network. The neural network has been used to build non-linear system models by Pantino and Liu [41], Chen and Xi [42], Hutchins [43], Antsaklis [44], Owens [45], Fernandez et al. [46] using many available experimental data sets. In particular, the Levenberg-Marquardt back-propagation algorithm is used for building the system model, Hagan and Menhaj [47]. The neural network back-propagation algorithm is applied in order to construct the entire CFCFB system model in this dissertation. The back-propagation algorithm uses tansig functions for the hidden layer and purelin functions for the output layer; therefore the data sets need to be normalized, using their minima and maxima. Simulation runs are performed for various step changes of the set points for both the bed-height and the SCR. 


\section{CHAPTER I. INTRODUCTION}

\subsection{Main Contributions of the Dissertation}

One of the main contributions of this dissertation is the building of a linear discrete system for estimating the solids circulation rate using a Kalman filter. With the least squares estimation and an extended observability matrix, the parameters $A, B$, and $C$, of the linear discrete state space system are estimated, Ljung [31]. Next, the system is transferred to another form without fictitious state variables that connect the input to output, then the output matrix, $H$ containing only the pressure components is introduced. The discrete linear system model uses measured pressure drops and the measured solids circulation rate so that the parameter, $A$, containing information about the solids circulation rate, as well as the pressure drops. Therefore, the linear discrete system model of the SCR works within a certain region. This system also suggests that the standpipe model is linear rather than non-linear in a given operating region.

The second contribution of this research is the estimation of the state and bed-height using a sliding mode observer. With the use of the previously developed standpipe dynamic model, Shim et al. [26], the collected pressure drops, the aeration flow rates, as well as the estimated solids circulation rate, the void fractions (state variables), and the bed-height are all estimated by the sliding mode observer. The sliding mode estimator uses the Lyapunov stability criteria, which states that if there exists a positive energy function and its derivative is negative then the system and/or estimator guarantees stability and convergence, Park et al. [48]. From the estimated void fractions, the bed-height can be calculated, since it separates the standpipe into two regions of low and high voidage.

The third contribution of this research is the calculation of the bed-height, using a 2-region method, which models the standpipe as two homogeneous regions, i.e. the lean and dense phases. This model uses three pressure drops, namely the lean phase, dense phase and total phase in the standpipe.

The final contribution of this research is the application of linear control theory to 
regulate the SCR and the bed-height. For simulation purposes, a neural network backpropagation algorithm is applied in order to model the entire CFCFB system, since there is no mathematical CFCFB system model available. The linear controller is used because there are linear relationships between the control variable (move air) and the SCR and/or the bed-height. 


\section{Chapter II}

\section{One Dimensional Dynamic Model of the Standpipe}

\subsection{Introduction}

In this chapter, a dynamic model of the standpipe in the CFCFB developed by Dr. N. W. Sams and Dr. E. J. Boyle, Shim et al. [26] is presented and discussed. The standpipe dynamic model of the CFCFB is critical to the implementation of an estimator and/or a controller. The standpipe dynamic model is based on conservation of mass, on the sum of the void fractions for gas and solid being one, and on Richardson and Zaki correlations. The state variable of the standpipe dynamic model is the gas void fraction. First, the standpipe is discretized into several cells in space. The discretized cell heights do not have to be equal in size. Second, the standpipe is assumed to be a one dimensional pipe in a z-axis, i.e. homogeneously distributed along the horizontal axes to make the system model simple and reliably fast. Third, the standpipe dynamic model uses the Richardson and Zaki correlation, Richardson and Zaki [49] to relate the gas void fraction to the relative speed, since the mass flow of the solids behaves as a flow of liquid. Finally, depending on 
the derivative of volumetric flux with respect to the void fraction at the discretized cell boundary, the volumetric flux is determined to be upward or downward. The measurement dynamic model is based on the Ergun equation, Coulson et al. [50], which relates the void fractions to the pressure drop profiles. The estimated pressure profiles are found to be an integrated version of the Ergun equation based on the discretized cells, using the trapezoidal rule.

\subsection{Standpipe Mathematical Model}

\subsubsection{The Conservation of Mass and Gas Flows}

An arbitrary control volume with a control surface, $A_{m}(\vec{r}, t)$, a density vector, $\rho(\vec{r}, t)$, a normal vector, $\vec{n}$, and a velocity vector, $\vec{w}$ is shown in Fig. 2.1. In this control volume, the total mass with a density function with respect to time and space can be found as,

$$
M(t)=\int_{V_{m}(t)} \rho_{s}(\vec{r}, t) \mathrm{d} V
$$

where $V_{m}(t)$ represents the volume that contains the mass, and $\rho_{s}(\vec{r}, t)$ is the density of the solid with respect to space and time. The time rate change of the total mass in the control volume can be found as,

$$
\frac{\mathrm{d} M(t)}{\mathrm{dt}}=\frac{\mathrm{d}}{\mathrm{dt}} \int_{V_{m}(t)} \rho_{s}(\vec{r}, t) \mathrm{d} V .
$$

Using the General Transport Theorem, the ordinary time derivative of the right hand side of (2.2) applying inside of the integrand can be found, but it should be a partial derivative plus the surface effect of the control surface as,

$$
\frac{\mathrm{d} M}{\mathrm{~d} t}=\frac{\mathrm{d}}{\mathrm{d} t} \int_{V_{m}(t)} \rho_{s}(\vec{r}, t) \mathrm{d} V=\int_{V_{m}(t)} \frac{\partial \rho_{s}(\vec{r}, t)}{\partial t} \mathrm{~d} V+\int_{A_{m}(t)} \rho_{s}(\vec{r}, t) \vec{w} \cdot \vec{n} \mathrm{~d} A .
$$




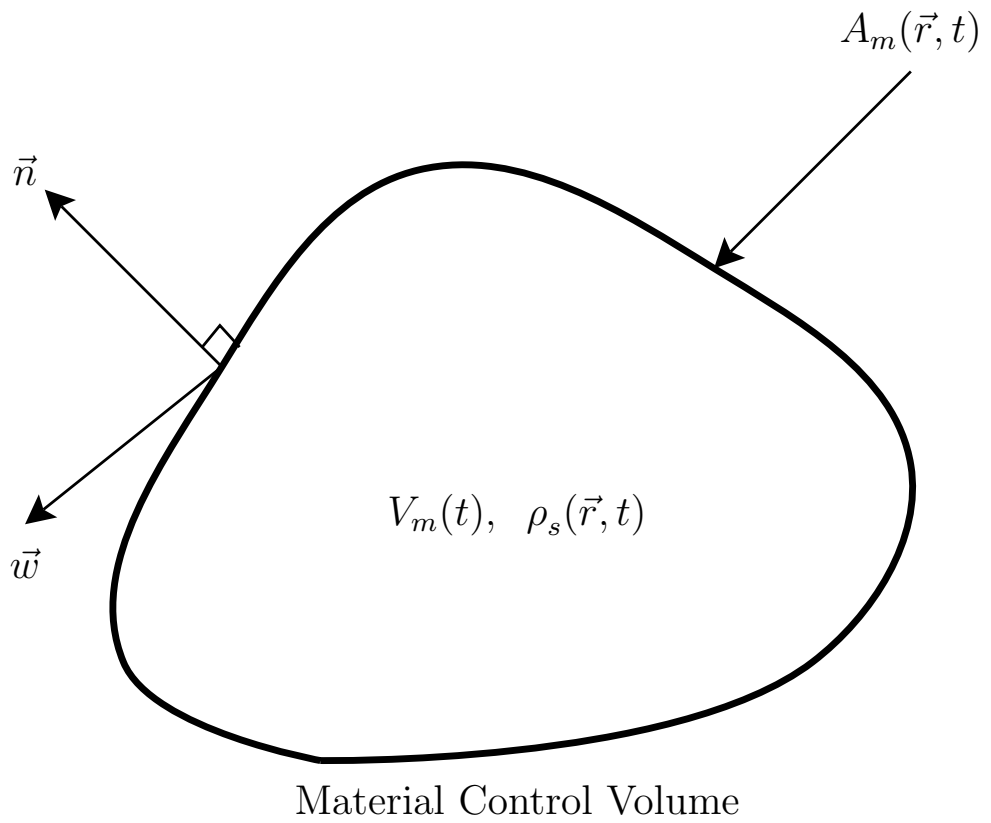

Figure 2.1: Control volume and its definitions of vertical surface, normal and speed vectors.

Therefore, the total time rate change of the total mass in the control volume is the sum of the solid density time rate change inside the control volume plus the transport in or out of the control surface. Furthermore, if the Divergence Theorem is utilized for the second term of (2.3), the total time rate change of the total mass, $\frac{\mathrm{d} M(t)}{\mathrm{dt}}$ becomes,

$$
\frac{\mathrm{d} M}{\mathrm{~d} t}=\int_{V_{m}(t)}\left(\frac{\partial \rho_{s}(\vec{r}, t)}{\partial t}+\nabla \cdot \rho_{s}(\vec{r}, t) \vec{w}\right) \mathrm{d} V,
$$

where $\nabla$ is the divergence operator, which calculates how much density is divergent in space. The total mass is assumed neither to be created nor destroyed in the control volume, so the time rate change of the total mass vanishes. From (2.4) and the fact that the time rate change of the total mass is zero, the continuity equation is derived as,

$$
0=\frac{\partial \rho_{s}(\vec{r}, t)}{\partial t}+\nabla \cdot \rho_{s}(\vec{r}, t) \vec{w}
$$

or,

$$
0=\frac{\partial \rho_{s}(\vec{r}, t)}{\partial t}+\left(\nabla \rho_{s}(\vec{r}, t)\right) \cdot \vec{w}+\rho_{s}(\vec{r}, t) \nabla \cdot \vec{w}
$$


However, the time derivative for the density with respect to time is,

$$
\frac{\mathrm{d} \rho_{s}(\vec{r}, t)}{\mathrm{d} t}=\frac{\partial \rho_{s}(\vec{r}, t)}{\partial t}+\left(\nabla \rho_{s}(\vec{r}, t)\right) \cdot \vec{w}
$$

Using (2.6), the continuity equation becomes,

$$
\frac{\mathrm{d} \rho_{s}(\vec{r}, t)}{\mathrm{d} t}+\rho_{s}(\vec{r}, t) \nabla \cdot \vec{w}=0 .
$$

\subsubsection{State Space Model of the Standpipe}

In the standpipe, the particle density, $\rho_{p}$ does not depend on either time or space. However, the aggregate density $\rho_{s}=\rho_{p} \times(1-\varepsilon)$ is a variable of interest, which may vary from place to place and from time to time. With the gas void fraction and (2.6), the following equation is obtained,

$$
\frac{\partial(1-\varepsilon)}{\partial t}+\nabla \cdot(1-\varepsilon) \vec{v}_{s}=0
$$

where $\varepsilon$ is the void fraction of the gas. Since the solid flux is measurable and the total flux (i.e. the flux sum of gas and solids) is constant in space, the solid velocity is replaced by the volumetric solid flux, $\vec{\jmath}_{s}=(1-\varepsilon) \vec{v}_{s}$, and continuity equation of the solids becomes, Shim et al. [26], Park et al. [11],

$$
\frac{\partial(1-\varepsilon)}{\partial t}+\nabla \cdot \vec{\jmath} s=0
$$

The same approach can be used for the gas flux. The continuity equation of the gas flux is now,

$$
\frac{\partial \varepsilon}{\partial t}+\nabla \cdot \vec{\jmath}_{g}=0
$$

and in this case, the relation, $\vec{\jmath}_{g}=\varepsilon \vec{v}_{g}$ is used. The total flux, $\vec{\jmath}_{T}(t) \equiv \vec{\jmath}_{s}(\vec{r}, t)+\vec{\jmath}_{g}(\vec{r}, t)$ is independent of the space through the standpipe and only depends on the time, so this total flux is used for dynamic equations. It is worth mentioning here that the solid flux is to be measured by the spiral, and the gas flux can be obtained since it is measured prior to 
being injected into the standpipe. Therefore, the total flux, $\vec{\jmath}_{T}(t)$ is assumed to be known in the dynamic model. The gas flux can be divided into upward and downward; however, the exact rates, i.e. how much is going up or down are not known. The relative velocity is given as,

$$
\vec{v}_{r}=\vec{v}_{g}-\vec{v}_{s}=\frac{\vec{\jmath}_{g}}{\varepsilon}-\frac{\vec{\jmath}_{s}}{1-\varepsilon}
$$

where $\vec{v}_{g}$ is the gas flow velocity and $\vec{v}_{s}$ is the solid flow velocity. The gas flux needs to be found in terms of the total flux, the gas void fraction, and the relative velocity. From $(2.11)$

$$
\varepsilon(1-\varepsilon) \vec{v}_{r}=(1-\varepsilon) \vec{\jmath}_{g}-\varepsilon \vec{\jmath}_{s},
$$

however, the gas flux can be written as $\vec{\jmath}_{s}=\vec{\jmath}_{T}-\vec{\jmath}_{g}$, and plugged into the above equation, then

$$
\varepsilon(1-\varepsilon) \vec{v}_{r}=(1-\varepsilon) \vec{\jmath}_{g}-\varepsilon\left(\vec{\jmath}_{T}-\vec{\jmath}_{g}\right)
$$

Arranging above equation, the gas flux is

$$
\vec{\jmath}_{g}=\varepsilon \vec{\jmath}_{T}+\varepsilon(1-\varepsilon) \vec{v}_{r}(\varepsilon) .
$$

In discretizing the space of the standpipe into small cells, a one dimensional space through $z$-axis is assumed. Therefore, the gradient of gas flux becomes a one dimensional partial differential equation and the continuity equation for the gas flux becomes,

$$
\frac{\partial \varepsilon}{\partial t}+\frac{\partial j_{g}(z, t)}{\partial z}=0
$$

For notational convenience, the subscript $g$ is now dropped. The partial differential equations for time and space can be discretized, using Euler approximation, Dorf and Bishop [51] as,

$$
\begin{aligned}
& \frac{\partial \varepsilon}{\partial t}=\frac{\varepsilon_{k}^{i}-\varepsilon_{k-1}^{i}}{\Delta t}, \\
& \frac{\partial j}{\partial z}=\frac{j_{k-1}^{i+}-j_{k-1}^{i-}}{\Delta z},
\end{aligned}
$$


where $\varepsilon_{k}^{i}$ is the void fraction in $i^{t h}$ cell at $k$ time, $\Delta t$ is the time propagation step, $j_{k-1}^{i+}$ is the gas volumetric flux from $i^{t h}$ to $(i+1)^{t h}$ at the previous time, $j_{k-1}^{i-}$ is the gas volumetric flux from $(i-1)^{t h}$ to $i^{\text {th }}$ cell at the previous time, and $\Delta z$ is the space propagation step. Replacing the partial differential equations with above Euler approximations yields,

$$
\frac{\varepsilon_{k}^{i}-\varepsilon_{k-1}^{i}}{\Delta t}+\frac{j_{k-1}^{i+}-j_{k-1}^{i-}}{\Delta z}=0
$$

rearranging (2.17) makes the discretized dynamic model,

$$
\varepsilon_{k}^{i}=\varepsilon_{k-1}^{i}+\frac{\Delta t}{\Delta z}\left(j_{k-1}^{i-}-j_{k-1}^{i+}\right)
$$

The gas flux from $(i-1)^{t h}$ to $i^{\text {th }}$ cell can be found using (2.14) because the total flux and void fractions at the previous time step are known. Some definitions make notation easy. Definition of the function $\zeta(\varepsilon)$ is,

$$
\zeta(\varepsilon) \equiv \varepsilon(1-\varepsilon) v_{r}(\varepsilon),
$$

and the derivative of function $\zeta(\varepsilon)$ with respect to $\varepsilon$ is,

$$
\zeta^{\prime}(\varepsilon) \equiv \frac{d \zeta(\varepsilon)}{d \varepsilon}
$$

with,

$$
v_{r}(\varepsilon)= \begin{cases}0 & \text { if } \varepsilon \leq \varepsilon_{p b}, \\ V_{t}\left(-a\left(\varepsilon-\varepsilon_{m f}\right)^{2}+\varepsilon_{m f}^{m-1}\right) & \text { if } \quad \varepsilon_{p b}<\varepsilon<\varepsilon_{m f}, \\ V_{t} \varepsilon^{n-1} & \text { if } \varepsilon \geq \varepsilon_{m f},\end{cases}
$$

where $a$ is $\frac{\varepsilon_{m f}^{n-1}}{\left(\varepsilon_{m f}-\varepsilon_{p b}\right)^{2}}, \varepsilon_{m f}$ is the void fraction at minimum fluidization, $\varepsilon_{p b}$ is the void fraction at a packed bed, and $V_{t}$ is the terminal velocity of the solid. The definition $v_{r}(\varepsilon)$ is obtained from the modified Richardson and Zaki correlation, Richardson and Zaki [49]. If the void fraction is below the packed bed, there is no flux developed (i.e. the first term of $\left.v_{r}(\varepsilon)\right)$. Moreover, there exists a connection between the packed bed condition and 
the fluidization condition (i.e. the second term of $v_{r}(\varepsilon)$ ). The last term of $v_{r}(\varepsilon)$ is the Richardson and Zaki correlation. The void fraction at a cell boundary is defined as the average of adjacent cells,

$$
\bar{\varepsilon}_{k-1}^{i-}=\frac{\varepsilon_{k-1}^{i-1}+\varepsilon_{k-1}^{i}}{2}
$$

and the total flux, $j_{T}$ is the algebraic sum of the gas and solid volumetric fluxes,

$$
j_{T}=j+j_{s}
$$

The wave speed $\lambda^{i-}$ of the void fraction disturbance at the boundary between $i-1$ and $i$ cells is given by Park et al. [11], Shim et al. [26],

$$
\lambda^{i-}=\left.\frac{j_{k-1}^{i-}}{\varepsilon}\right|_{\varepsilon=\bar{\varepsilon}_{k-1}^{i-}}=j_{T}+\zeta^{\prime}\left(\bar{\varepsilon}_{k-1}^{i-}\right) .
$$

The wave speed propagates either up or down at the $i^{\text {th }}$ cell in the standpipe and the gas flux is determined according to this wave speed. If the wave speed is upward $\left(\lambda^{i-}>0\right)$, then the gas flux is,

$$
j_{k-1}^{i-}=j_{T} \varepsilon_{k-1}^{i-1}+\zeta\left(\varepsilon_{k-1}^{i-1}\right)
$$

and if the wave speed downward $\left(\lambda^{i-}<0\right)$, then the gas flux is,

$$
j_{k-1}^{i-}=j_{T} \varepsilon_{k-1}^{i}+\zeta\left(\varepsilon_{k-1}^{i}\right)
$$

A detailed diagram of the system model propagation is shown in Fig. 2.2. The volumetric fluxes are obtained from the void fraction at the previous time and space steps. The current void fraction is calculated from these volumetric fluxes depending on the wave speed at the boundaries. At time $k-1$, the boundary condition, $\varepsilon_{k-1}^{i-}$ on $i-1$ and $i$ cells is first found and according to this boundary condition, the wave speed, $\lambda^{i-}$ is calculated. In addition, the volumetric flux, $j_{k-1}^{i-}$ is obtained according to the wave speed signs. This calculation also needs to be obtained for the $i$ and $i+1$ cell to calculate volumetric flux, $j_{k-1}^{i+}$. From these two volumetric fluxes, the current void fraction on the $i^{\text {th }}$ cell can be updated, using the standpipe dynamic equation. 


\subsection{Standpipe Measurement Model}

The measurements in the standpipe of the CFCFB are the pressure drop profiles between the several points and the topmost point. Since the state variables are the gas void fraction and the measurements are the pressure drop profiles, the measurement dynamic model should relate the void fraction to the pressure drop profiles. A detailed diagram of

Time

$\begin{array}{cccc} & k-1 & k & k+1 \\ i+1 & \circ & \circ & \circ\end{array}$

$\uparrow$

$i+$

Space

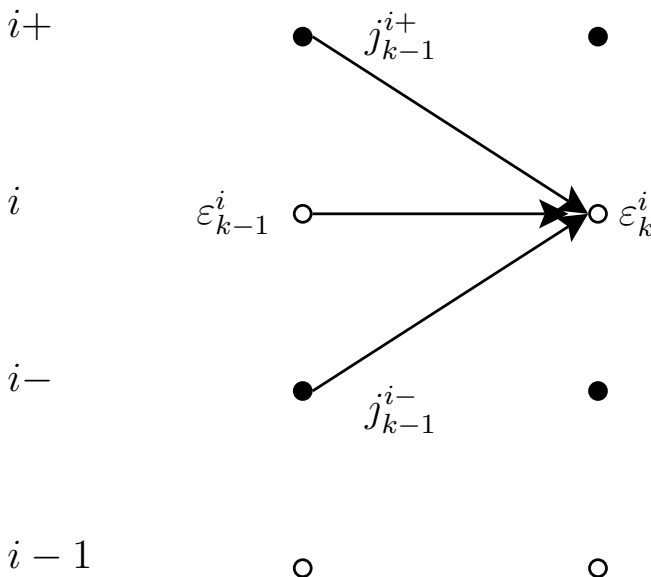

$i-1$ 
relates the void fractions to the pressure drop profile in one discretized cell, Shim et al. [26], Coulson et al. [50]. The Ergun equation is given by Coulson et al. [50],

$$
\frac{\Delta P}{L}=150 \frac{(1-\varepsilon)^{2}}{\varepsilon^{3}} \frac{\mu u_{c}}{d^{2}}+1.75 \frac{1-\varepsilon}{\varepsilon^{3}} \frac{\rho_{s} u_{c}^{2}}{d},
$$

where $\Delta P$ is the pressure drop, $L$ is the difference where the pressure drop is measured, $\mu$ is the fluid viscosity, $u_{c}$ is the interstitial velocity defined as $\varepsilon v_{r}(\varepsilon)$, and $d$ is the effective particle diameter. For the CFCFB, the pressure drop profile is used for the measurements,

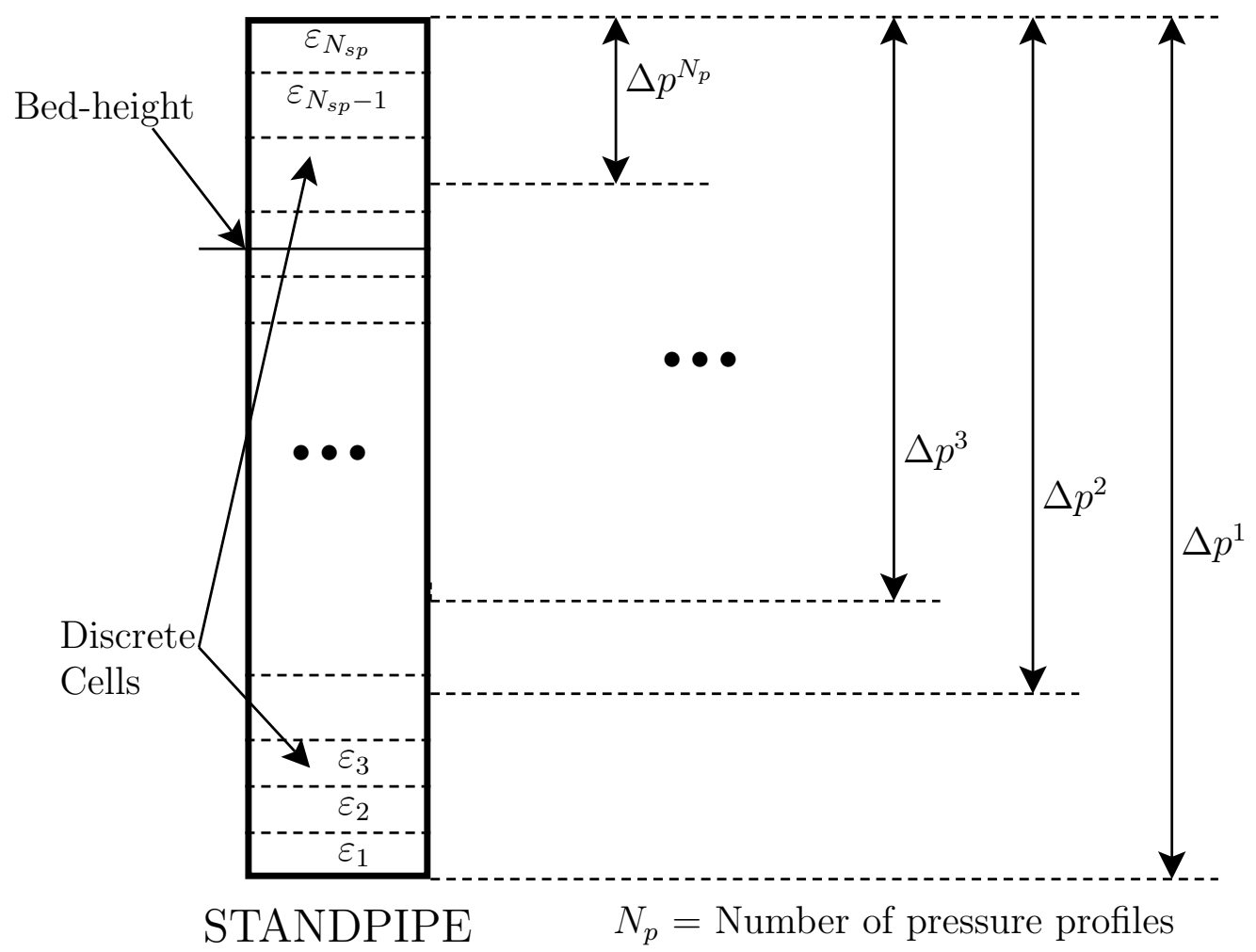

Figure 2.3: The measurement diagram of the standpipe.

so the Ergun equation has to be integrated through the z-axis using the trapezoidal rule. The pressure drop for $i^{\text {th }}$ cell for the standpipe of the CFCFB is given as,

$$
\Delta p^{i}=\left(z_{i+1}-z_{i}\right) \times\left(C_{1} v_{r}\left(\frac{1-\varepsilon^{i}}{\varepsilon^{i}}\right)^{2}+C_{2}\left(\frac{1-\varepsilon^{i}}{\varepsilon^{i}}\right) v_{r}\left|v_{r}\right|\right),
$$


where $C_{1}$ is a constant $\frac{150 \mu}{d^{2}}, \mu$ is the fluid viscosity, $d$ is the effective particle diameter, and $C_{2}$ is a constant $\frac{1.75 \rho_{g}}{d}, \rho_{g}$ is gas density. The pressure profile can be found from the integrated form as,

$$
p_{k}^{n}=\sum_{i=n}^{N_{s p}-1} \frac{p_{k}^{i} \times \Delta z_{i-1}+p_{k}^{i+1} \times \Delta z_{i}}{\Delta z_{i-1}+\Delta z_{i}},
$$

where $\Delta z_{i}$ is $z_{i+1}-z_{i}$, and $N_{s p}$ is the number of cells in the standpipe. In cases in which the pressure tap is not matched to the discretized cell position, the integration should be modified from the beginning of the cell. 


\section{Chapter III}

\section{Solids Circulation Rate and Bed-height Estimations}

\subsection{Introduction}

A solids circulation rate (SCR) is one of the critical components in improving the operation of the system, in estimating the states and the bed-height, and in using estimator methods such as those involving an extended Kalman filter, an $\mathcal{H}_{\infty}$ methods, Park et al. [11], Shim et al. [26], or a sliding mode estimator as will be discussed in Chapter IV. Moreover, the SCR is one of the important parameters in the operation of the CFB, since it affects mass and heat transfer characteristics, which in turn impact the efficiency of the processes. Although some techniques have been used to determine this variable, notably Ludlow et al. [13], Burkell et al. [27], Lui and Bowen [28], the measurement of this parameter in industrial-scale CFB units operating under extreme processing conditions is very difficult. Thus, to be practical, there is a need for an estimator of the solids circulation rate that uses only the pressure drops and move air information available from the measurements. 


\section{CHAPTER III. SCR AND BED-HEIGHT ESTIMATES}

The SCR can be measured from the spiral installed at a point $2.7432 \mathrm{~m}$ from the bottom of the standpipe of the CFCFB at NETL, in Morgantown, WV. In this chapter, the system model for the purpose of SCR estimation is developed, Ljung [31]. The system model is defined as,

$$
\begin{aligned}
x(k+1) & =A x(k)+B u(k), \\
y(k) & =C x(k),
\end{aligned}
$$

where $x(k)$ is the fictitious state vector, $u(k)$ is the input vector, $y(k)$ is the output vector, and $A, B$, and $C$ are constant matrices. Least squares approximation methods, Brotherton and Caines [32] and an extended observability matrix through a subspace algorithm are applied in order to estimate the matrices $A, B$, and $C$, Park et al. [12]. These methods will be discussed in the next few sections. Once the system model has been built, a Kalman filter algorithm is utilized in order to estimate the solids circulation rate. The experimental results show the effectiveness of this method.

The bed-height of the standpipe is another important factor in terms of operating and controlling the CFCFB. This measurement could indicate whether the system is running in the operable regions or in the efficient regions. In this section, the bed-height calculation method with a two-region model using information from three pressure drops is introduced and discussed. The standpipe can be modeled into two homogeneous sections separated by the bed-height. The upper part is called the lean or dilute region (i.e. the high void fraction) because there are not many solids while lower part is called the dense or fluidized region (i.e. the low void fraction) because many solids flow in the region. Using three types of pressure information, i.e. lean, dense, and total region, the bed-height can be obtained by simple calculations. The simulation results show that this method is fast and effective. 


\subsection{Preliminaries}

An auto regressive moving average (ARMA) method can be used to build the dynamic model where the massive data sets are available, but the exact mathematical model cannot be built directly, Amjady [52], Chow and Tan [53], Forssell and Ljung. [54]. The following section describes the basic concepts of the time series used for the system model development of the solids circulation rate.

\subsubsection{Basic Theory}

A basic theoretical explanation for some concepts is presented in this section to assist the reader in understanding the theory behind developing a system model of the SCR. Let us assume the existence of a time series, $z_{t}$. The mean value, $\mu$ is theoretically integrated as, Box and Jenkins. [55], Shumway and Stoffer [56],

$$
\mu=E\left[z_{t}\right] \equiv \int_{-\infty}^{\infty} z p(z) d z,
$$

where $p(z)$ is a probability function with respect to a certain point in the time series, $z_{t}$. In practice, however, the theoretical mean value cannot be obtained exactly with this integral equation because the mathematical model usually is not available. Therefore, the approximated mean value is alternatively considered,

$$
\mu \approx \bar{z} \equiv \frac{1}{N_{t}} \sum_{t=1}^{N_{t}} z_{t},
$$

where $N_{t}$ is the total number of the time series, $z_{t}$. Next, the auto-covariance matrix for time series $z_{t}$ at lag $\mathrm{k}$ is defined as,

$$
\gamma_{k} \equiv \operatorname{cov}\left[z_{t}, z_{t+k}\right]=E\left[\left(z_{t}-\mu\right)\left(z_{t+k}-\mu\right)\right]
$$

On the other hand, the auto-correlation is given as,

$$
\rho_{k} \equiv \frac{\gamma_{k}}{\gamma_{0}}
$$


Like the mean value, the practical auto-correlation function can be approximated to a partial auto-correlation by,

$$
r_{k}=\frac{c_{k}}{c_{0}},
$$

where,

$$
c_{k} \equiv \frac{1}{N_{t}} \sum_{t=1}^{N_{t}-k}(z-\bar{z})\left(z_{t+k}-\bar{z}\right), k=0,1,2, \ldots, K .
$$

The stationary processes are not affected by a change of time origin, and in a particular state of statistical equilibrium, they have a constant mean, or are fluctuating around their means. The white noises are uncorrelated, so the auto-correlation of the white noise can be found as,

$$
\rho_{k}=\left\{\begin{array}{cc}
1 & k=0 \\
0 & k \neq 0
\end{array}\right.
$$

\section{General Linear Process}

There are two equivalent forms for the linear process. The first one is given by

$$
\begin{aligned}
\tilde{z}_{t} & \equiv a_{t}+\psi_{1} a_{t-1}+\psi_{2} a_{t-2}+\cdots, \\
& =a_{t}+\sum_{j=1}^{\infty} \psi_{j} a_{t-j}
\end{aligned}
$$

where $\tilde{z}_{t}=z_{t}-\mu$. The other form can be described as the series weighted sums of the past values of the $\tilde{z}$ 's, plus the added noise, $a_{t}$,

$$
\begin{aligned}
\tilde{z}_{t} & =\pi_{1} \tilde{z}_{t-1}+\pi_{2} \tilde{z}_{t-2}+\cdots+a_{t}, \\
& =\sum_{j=1}^{\infty} \pi_{j} \tilde{z}_{t-j}+a_{t} .
\end{aligned}
$$

The relationships between the $\psi$ weights and $\pi$ weights can be found using the backward shift operator

$$
B z_{t}=z_{t-1}, B^{j} z_{t}=z_{t-j} .
$$


Now, consider the expressions $(1-\theta B)^{-1} \tilde{z}_{t}=a_{t}$. For $|\theta|<1$, the left hand side can be expanded to

$$
\left(1+\theta B+\theta^{2} B^{2}+\theta^{2} B^{2}+\cdots\right) \tilde{z}_{t}=a_{t}
$$

From the above equation, the deviation $\tilde{z}_{t}$ can be expressed in terms of the previous $\tilde{z}_{t}$ with $a_{t}$,

$$
\tilde{z}_{t}=-\theta \tilde{z}_{t-1}-\theta^{2} \tilde{z}_{t-2}-\cdots+a_{t}
$$

so that for this model $\pi_{j}=-\theta^{j}$.

From (3.10), another expression can be found

$$
\left(1-\sum_{j=1}^{\infty} \pi_{j} B^{j}\right) \tilde{z}_{t}=a_{t},
$$

or,

$$
\pi(B) \tilde{z}_{t}=a_{t}
$$

with

$$
\pi(B)=1-\sum_{j=1}^{\infty} \pi_{j} B^{j} .
$$

Multiplying $\psi B$ on both side of (3.14) yields,

$$
\psi(B) \pi(B) \tilde{z}_{t}=\psi(B) a_{t}=\tilde{z}_{t},
$$

hence,

$$
\psi(B) \pi(B)=1,
$$

therefore,

$$
\pi(B)=\psi^{-1}(B) .
$$

\subsubsection{Least Squares Approximation}

The least squares method indicates that the best fit can be obtained by minimizing the sum of squares of the difference between the given (measured) points and the estimated 


\section{CHAPTER III. SCR AND BED-HEIGHT ESTIMATES}

curves, Haykin [57]. In this section, the least square method applied in order to build the SCR system model is introduced and discussed, Ljung [31], Haykin [57]. The least squares method is used to determine parameters for the model based system, such as the AR or ARMA model. The linear regression is defined as,

$$
\hat{y}(t \mid \theta)=\psi^{T}(t) \theta+\mu(t)
$$

where $\theta$ is the regression vector (parameters), $\psi(t)$ is the previously known input and output data vector, and $\mu(t)$ is the noise vector. The error equation based on (3.19) is,

$$
e(t, \theta)=y(t)-\psi^{T}(t) \theta
$$

where $y(t)$ is a measured data vector. In a least squares estimator, the criterion of a predictor is given by the error square and divides the sum of the all possible terms as,

$$
V_{N_{t}}\left(\theta, Z^{N_{t}}\right)=\frac{1}{N_{t}} \sum_{t=1}^{N_{t}} \frac{1}{2} e(t, \theta)^{2} .
$$

With the substitution of (3.20) for the error term, the criterion of the predictor is,

$$
V_{N_{t}}\left(\theta, Z^{N_{t}}\right)=\frac{1}{N_{t}} \sum_{t=1}^{N_{t}} \frac{1}{2}\left[y(t)-\psi^{T}(t) \theta\right]^{2} .
$$

The criterion, (3.22), needs to be minimized with respect to parameters $\theta$ according to the state of the least squares method. This criterion is a quadratic function of the parameters $\theta$, so it can be minimized analytically,

$$
\hat{\theta}_{N_{t}}=\left[\frac{1}{N_{t}} \sum_{t=1}^{N_{t}} \psi(t) \psi^{T}(t)\right]^{-1} \frac{1}{N_{t}} \sum_{t=1}^{N_{t}} \psi(t) y(t) .
$$

The vectors $y(t)$ and $\phi(t)$ are known from the input and output vectors, so the parameter vector $\theta$ can be determined from the known experimental data. 


\subsection{SCR System Development}

\subsubsection{Subspace Algorithm}

To estimate the state space model parameters $A, B$, and $C$, the least squares estimator technique and an extended observability matrix are used through the subspace algorithm, Ljung [31]. The system equation defined in (3.1) is rewritten with some noise terms as,

$$
\begin{array}{ll}
x(t+1) & =A x(t)+B u(t)+w(t), \\
y(t) & =C x(t)+v(t),
\end{array}
$$

where $x(t)$ is the fictitious state vector, $u(t)$ is the input vector, $y(t)$ is the measurement vector, $w(t)$ and $v(t)$ are noise terms, and $A, B$, and $C$ are constant matrices to be determined. Define an extended observability matrix (EOM) as,

$$
O_{r}=\left[C, C A, C A^{2}, \cdots, C A^{n-1}\right]^{T},
$$

where $n$ is the dimension of matrix $A$. At this point, if the base is changed with an arbitrary invertible matrix $T$, the system equation becomes,

$$
\begin{array}{ll}
\tilde{x}(t+1) & =T^{-1} A T \tilde{x}(t)+T^{-1} B u(t)+\tilde{w}(t), \\
y(t) & =C T \tilde{x}(t)+v(t) .
\end{array}
$$

The extended observability matrix, $O_{r}$ also needs to be changed using the invertible matrix $T$, yielding,

$$
\tilde{O}_{r}=O_{r} T \text {. }
$$

In (3.27), since multiplying the extended observability matrix from right by any invertible matrix does not change the system dynamic matrix, this is just the basis change of representation, Ljung [31]. Therefore, to obtain the system dynamic model, any extended observability matrix can be used on any basis. In fact, the changing basis does not affect 


\section{CHAPTER III. SCR AND BED-HEIGHT ESTIMATES}

the original system matrices, $A, B$, and $C$. The defined system equation, (3.24) leads to,

$$
\begin{aligned}
y(t+k) & =C A^{k} x(t)+C A^{k-1} B u(t)+\cdots+C B u(t+k-1) \\
& +C A^{k-1} w(t)+C A^{k-2} w(t+1)+\cdots+C w(t+k-1)+v(t+k) .
\end{aligned}
$$

Rewriting (3.28) in a vector form,

$$
Y_{r}(t)=O_{r} x(t)+S_{r} U_{r}(t)+V(t)
$$

where,

$$
Y_{r}(t)=\left[\begin{array}{c}
y(t) \\
y(t+1) \\
\vdots \\
y(t+r-1)
\end{array}\right], U_{r}(t)=\left[\begin{array}{c}
u(t) \\
u(t+1) \\
\vdots \\
u(t+r-1)
\end{array}\right], \quad S_{r}=\left[\begin{array}{ccc}
D & \cdots & 0 \\
C B & \cdots & 0 \\
\vdots & \ddots & \vdots \\
C A^{r-2} B & \cdots & D
\end{array}\right],
$$

$V(t)$ contains the noise terms and $O_{r}$ is the extended observability matrix defined in (3.25).

For the convenience of notation, the matrix form is applied here,

$$
\mathbf{Y}=O_{r} \mathbf{X}+S_{r} \mathbf{U}+\mathbf{V}
$$

where,

$$
\begin{aligned}
\mathbf{Y} & =\left[Y_{r}(1), Y_{r}(2), \cdots, Y_{r}(N)\right], \\
\mathbf{X} & =[x(1), x(2), \cdots, x(N)], \\
\mathbf{U} & =\left[U_{r}(1), U_{r}(2), \cdots, U_{r}(N)\right], \\
\mathbf{V} & =\left[V_{r}(1), V_{r}(2), \cdots, V_{r}(N)\right] .
\end{aligned}
$$

From (3.30), it is necessary to find the extended observability matrix, $O_{r}$ in terms of the measured input and output matrices. The idea of finding the extended observability matrix is to eliminate the input and noise terms using known input and output matrices. First, remove the input term multiplying by $\Pi=\mathbf{I}-\mathbf{U}^{T}\left(\mathbf{U U}^{T}\right)^{-1} \mathbf{U}$,

$$
\begin{aligned}
\mathbf{Y} \Pi & =O_{r} \mathbf{X} \Pi+S_{r} \mathbf{U} \Pi+\mathbf{V} \Pi, \\
& =O_{r} \mathbf{X} \Pi+\mathbf{V} \Pi,
\end{aligned}
$$


since

$$
\mathbf{U} \Pi=\mathbf{U}-\mathbf{U U}^{T}\left(\mathbf{U U}^{T}\right)^{-1} \mathbf{U}=0 .
$$

Next, it is necessary to remove the noise term so that the extended observability matrix can be determined. Assume that noise terms can be made to be vanished by right multiplying a matrix, $\Phi$,

$$
G \equiv \frac{1}{N} \mathbf{Y} \Pi \Phi^{T}=\frac{1}{N} O_{r} \mathbf{X} \Pi \Phi^{T}+\frac{1}{N} \mathbf{V} \Pi \Phi^{T}
$$

therefore,

$$
G \equiv \frac{1}{N} O_{r} \mathbf{X} \Pi \Phi^{T}
$$

where $\Phi=[\phi(1), \phi(2), \cdots, \phi(N)]$. Expanding the noise term with components yields,

$$
\begin{aligned}
\frac{1}{N} \mathbf{V} \Pi \Phi^{T}= & \frac{1}{N} \sum_{t=1}^{N} V(t) \phi^{T}(t)-\frac{1}{N} \sum_{t=1}^{N} V(t) U_{r}^{T}(t) \\
& \times\left[\frac{1}{N} \sum_{t=1}^{N} U_{r}(t) U_{r}^{T}(t)\right]^{-1} \frac{1}{N} \sum_{t=1}^{N} U_{r}(t) \phi^{T}(t) .
\end{aligned}
$$

With the large number of $N$, (3.35) becomes

$$
\lim _{N \rightarrow \infty} \frac{1}{N} \mathbf{V} \Pi \Phi^{T}=E\left[V(t) \phi^{T}\right]-E\left[V(t) U_{r}^{T}(t)\right] R_{U}^{-1} E\left[U_{r}(t) \phi^{T}(t)\right],
$$

where $R_{U}(t)=E\left[U_{r}(t) U_{r}^{T}(t)\right]$. To make this term vanish, it is necessary to choose the right matrix, $\Phi$. Since the measurements and inputs are all known, the vector, $\phi(t)$ is determined to be,

$$
\phi(t)=\left[y(t-1), \cdots, y\left(t-s_{1}\right), u(t-1), \cdots, u\left(t-s_{2}\right)\right]^{T},
$$

where $s_{1}$ and $s_{2}$ are large numbers. Thus the expected values between the noise term and this $\phi$ vector vanish as does the noise term. From (3.33), the following is derived,

$$
G=O_{r} \frac{1}{N} \mathbf{X} \Pi \Phi^{T} \equiv O_{r} \tilde{T}_{N}
$$

where $\tilde{T}_{N} \equiv \frac{1}{N} \mathbf{X} \Pi \Phi^{T}$ is just a matrix to change the basis. With the weighting matrices in the singular value decomposition, the following is obtained,

$$
\hat{G}=W_{1} G W_{2}=U S V^{T} \sim U_{1} S_{1} V_{1}^{T}
$$


A full rank matrix $R$ is selected, making the estimated observable matrix, $\hat{O}_{r}=W_{1}^{-1} U_{1} R$. Finally, the equations are solved by the least squares method,

$$
\begin{aligned}
\hat{C} & =\hat{O}_{r}\left(1: N_{p}, 1: n\right), \\
\hat{O}_{r}\left(N_{p}+1: p r, 1: n\right) & =\hat{O}_{r}\left(1: N_{p}(r-1), 1: n\right) \hat{A}
\end{aligned}
$$

where $N_{p}$ is a dimension of the output vector, $y(t)$ and $n$ is the dimension of the system state vector, $x(t)$. The matrix $\hat{B}$ is estimated using the least squares like method from,

$$
\min _{B} \frac{1}{N_{e}} \sum_{t=1}^{N}\left\|y(t)-\hat{C}(q I-\hat{A})^{-1} B u(t)\right\|^{2} .
$$

There are several methods available for choosing weighting matrices, $W_{1}, W_{2}$ and $R$, Ljung [31]. The technique applied in this research uses the methods "N4SID" proposed by Van Overschee and DeMoor (1994), Ljung [31] where the weighting matrices are chosen as,

$$
\begin{aligned}
& W_{1}=I, \\
& W_{2}=\left(\frac{1}{N_{e}} \Phi \prod \frac{1}{U^{T}} \Phi^{T}\right)^{-1} \Phi .
\end{aligned}
$$

\subsubsection{SCR Estimator Development}

As described in the previous section, an effective system dynamic model for estimating the solids circulation rate is built, using a least squares estimation and the extended observability matrix, Ljung [31]. Once the matrices $A, B$, and $C$ of the system dynamic model have been determined, a Kalman filter algorithm is applied in order to estimate the solids circulation rate with error corrections from the measured pressure drop profiles. First, the system dynamic model is given as,

$$
\begin{aligned}
x_{f}(t+1) & =\bar{A} x_{f}(t)+\bar{B} u(t), \\
x(k) & =C x_{f}(t),
\end{aligned}
$$

where $x_{f}(t)$ is the fictitious state, $u(t)$ is the input vector, $x(t)$ is the output vector, and $\bar{A}, \bar{B}, C$ are the constant matrices to be determined. The details of input and output 
name tags for building the system dynamic model are shown in Table 3.1. Furthermore, the operating conditions of the CFCFB for building the SCR system model are shown in Table 3.2. There are several other conditions, but here a few of the major operating conditions are shown. The challenge of using this method is to determine the matrices

\begin{tabular}{c|c||c|c}
\hline \hline Output & Components & Input & Components \\
\hline$x_{1}(t)$ & pressure from $0.01 \mathrm{~m}$ to top of standpipe & $u_{1}(t)$ & move air \\
\hline$x_{2}(t)$ & pressure from $1.13 \mathrm{~m}$ to top of standpipe & $u_{2}(t)$ & riser aeration \\
\hline$x_{3}(t)$ & pressure from $2.35 \mathrm{~m}$ to top of standpipe & $u_{3}(t)$ & total pressure of riser \\
\hline$x_{4}(t)$ & pressure from $2.96 \mathrm{~m}$ to top of standpipe & & \\
\hline$x_{5}(t)$ & pressure from $3.48 \mathrm{~m}$ to top of standpipe & & \\
\hline$x_{6}(t)$ & pressure from $4.69 \mathrm{~m}$ to top of standpipe & & \\
\hline$x_{7}(t)$ & pressure from $6.34 \mathrm{~m}$ to top of standpipe & & \\
\hline$x_{8}(t)$ & pressure from $8.2 \mathrm{~m}$ to top of standpipe & & \\
\hline$x_{9}(t)$ & measured solids circulation rate & & \\
\hline
\end{tabular}

Table 3.1: Detailed input and output components.

$\bar{A}, \bar{B}, C$. The least squares approximation estimation and the extended observability matrix are used to estimate the matrices, $\bar{A}, \bar{B}$, and $C$ with the collected data from the CFCFB as explained in Section 3.3. Once these matrices are determined, the system dynamic model is transfered to a different format with no fictitious states, using $x_{f}(t)=C^{-1} x(t)$ as,

$$
x(t+1)=A x(t)+B u(t),
$$

where $A=C \bar{A} C^{-1}$ and $B=C \bar{B}$. The fictitious state $x_{f}(t)$ is eliminated and only pressure drop profiles and the SCR are now contained in the state vector, $x(t)$. The measurement vector contains nothing but the pressure drop profiles, so the state output in the system model should not contain the solids circulation rate but only the pressure drop profiles. The new matrix, $H$ and new output vector, $y(t)$ are introduced to fulfill these needs. The system model now becomes,

$$
\begin{aligned}
x(t+1) & =A x(t)+B u(t), \\
y(t) & =H x(t) .
\end{aligned}
$$


CHAPTER III. SCR AND BED-HEIGHT ESTIMATES

\begin{tabular}{|c|c|c|c|c|c|c|c|}
\hline Name Tag & \multicolumn{6}{|c|}{ Properties } & Units \\
\hline Bed Material & \multicolumn{6}{|c|}{ Corks $\left(d=829 \mu, \rho_{s}=189\right)$} & $\mathrm{m}, \mathrm{kg} / \mathrm{m}^{3}$ \\
\hline$U_{g}$ & \multicolumn{6}{|c|}{5.27} & $\mathrm{~m} / \mathrm{sec}$ \\
\hline$F_{m}$ (Move Air) & 1430 & 1426 & 1416 & 765 & 764 & 739 & $\mathrm{SCFH}$ \\
\hline Periods & 90 & 60 & 120 & 60 & 90 & 120 & sec \\
\hline Inventory Solids & \multicolumn{6}{|c|}{68} & $\mathrm{~kg}$ \\
\hline Humidity & \multicolumn{6}{|c|}{90} & $\%$ \\
\hline
\end{tabular}

Table 3.2: Operating conditions of the CFCFB for building the SCR system model.

Based on the system model, (3.46), the suggested estimator with the Kalman filter algorithm is defined as,

$$
\begin{aligned}
\hat{x}(t+1) & =A \hat{x}(t)+B u(t)+K(p(t)-\hat{y}(t)), \\
\hat{y}(t) & =H \hat{x}(t),
\end{aligned}
$$

where $(\hat{.})$ indicates the estimated state or output vector, $p(t)$ is the measured pressure drop profiles, and $K$ is the Kalman gain matrix found from the Kalman filter algorithm, which is discussed in the next section.

\subsection{Kalman Filter Algorithm}

Since the dynamic system model is in linear form, a discrete linear Kalman filter algorithm is discussed here, Gelb [58]. The system and measurement models are defined as,

$$
\begin{aligned}
& x_{k}=\Phi_{k-1} x_{k-1}+w_{k-1}, \\
& z_{k}=H_{k} x_{k}+v_{k},
\end{aligned}
$$

where $\Phi_{k-1}$ is the discrete time variant state matrix, $H_{k}$ is the time variant measurement matrix, while $w_{k-1}$ and $v_{k}$ are white noise and have constant covariance. Several assumptions need to be considered to build the discrete linear Kalman filter algorithm. First, the noise for the state and measurement is assumed to be white and has a constant covariance. Second, the covariance matrices, $Q$ and $R$ have to be chosen in the beginning of 


\section{CHAPTER III. SCR AND BED-HEIGHT ESTIMATES}

the estimation procedure. Third, the estimator is unbiased, i.e. $E\left[\tilde{x}_{k}\right]=0$ at any time. Finally, the estimator is not correlated to the processing and measurement noises, i.e. $E\left[x_{k} w_{k-1}\right]=E\left[x_{k} v_{k}\right]=0$. To save computer memory and reduce the number of variables, the discrete linear Kalman filter uses the recursive filtering procedure.

\subsubsection{Derivation of a Discrete Linear Kalman Filter}

First, the recursive filter is utilized to save computer memory while the estimation is being performed, Gelb [58],

$$
\hat{x}_{k}(+)=K_{k}^{\prime} \hat{x}_{k}(-)+K_{k} z_{k}
$$

where $K_{k}^{\prime}$ and $K_{k}$ are the matrices to be determined, $z_{k}$ is a measurement vector, $\hat{x}_{k}(+)$ is a posterior estimated vector, and $\hat{x}_{k}(-)$ is a prior estimated vector. Derivation procedure starts with a prior and a posterior error definitions (the tilde denotes estimation error),

$$
\begin{aligned}
& \tilde{x}_{k}(+)=\hat{x}_{k}(+)-x_{k}, \\
& \tilde{x}_{k}(-)=\hat{x}_{k}(-)-x_{k} .
\end{aligned}
$$

Using a recursive filter, (3.49), error equations, (3.50), and the system equation, (3.48) the posterior error becomes,

$$
\tilde{x}_{k}(+)=K_{k}^{\prime}\left(\tilde{x}_{k}(-)+x_{k}\right)+K_{k}\left(H_{k} x_{k}+v_{k}\right)-x_{k},
$$

rearranging the above equation results in,

$$
\tilde{x}_{k}(+)=\left[K_{k}^{\prime}+K_{k} H_{k}-I\right] x_{k}+K_{k}^{\prime} \tilde{x}_{k}(-)+K_{k} v_{k}
$$

Taking the expected operator to (3.52) yields,

$$
E\left[\tilde{x}_{k}(+)\right]=\left[K_{k}^{\prime}+K_{k} H_{k}-I\right] E\left[x_{k}\right]+K_{k}^{\prime} E\left[\tilde{x}_{k}(-)\right]+K_{k} E\left[v_{k}\right]
$$

The left hand side of the equation (3.53) vanishes according to the unbiased estimator, and with the white measurement noise the last term of the right hand side also vanishes. The 


\section{CHAPTER III. SCR AND BED-HEIGHT ESTIMATES}

second term of the right hand side is also zero because the estimator is unbiased, so the first term of the right hand side should be zero to satisfy (3.53). Therefore, the time-varying weighting matrix, $K_{k}^{\prime}$ is required to be,

$$
K_{k}^{\prime}=I-K_{k} H_{k}
$$

Using (3.54), makes the recursive estimator (3.49),

$$
\hat{x}_{k}(+)=\hat{x}_{k}(-)+K_{k}\left[z_{k}-H_{k} \hat{x}_{k}(-)\right] .
$$

Next, the error covariance update needs to be found, based on the estimator, (3.55). By definition, the posterior error covariance matrix is,

$$
P_{k}(+)=E\left[\tilde{x}_{k}(+) \tilde{x}_{k}(+)^{T}\right] .
$$

The posterior state error is,

$$
\tilde{x}_{k}(+)=\hat{x}_{k}(+)-x_{k},
$$

and using (3.55), the posterior state error can be rewritten as,

$$
\begin{aligned}
\tilde{x}_{k}(+) & =\hat{x}_{k}(-)+K_{k}\left[z_{k}-H_{k} \hat{x}_{k}(-)\right]-x_{k} \\
& =\tilde{x}_{k}(-)+K_{k}\left(H_{k} x_{k}+v_{k}-H_{k} \tilde{x}_{k}(-)-H_{k} x_{k}\right) \\
& =\tilde{x}_{k}(-)-K_{k} H_{k} \tilde{x}_{k}(-)+K_{k} v_{k} .
\end{aligned}
$$

Rearranging above equation makes the posterior state error equation,

$$
\tilde{x}_{k}(+)=\left(I-K_{k} H_{k}\right) \tilde{x}_{k}(-)+K_{k} v_{k}
$$

With (3.59), the posterior error covariance matrix becomes,

$$
P_{k}(+)=E\left[\left(I-K_{k} H_{k}\right) \tilde{x}_{k}(-)+K_{k} v_{k}\right]\left[\left(I-K_{k} H_{k}\right) \tilde{x}_{k}(-)+K_{k} v_{k}\right]^{T} .
$$

Expanding above equation yields,

$$
\begin{aligned}
P_{k}(+)= & E\left[\left(I-K_{k} H_{k}\right) \tilde{x}_{k}(-) \tilde{x}_{k}(-)^{T}\left(I-K_{k} H_{k}\right)^{T}+\left(I-K_{k} H_{k}\right) \tilde{x}_{k}(-) v_{k}^{T} K_{k}^{T}+\right. \\
& \left.K_{k} v_{k} \tilde{x}_{k}(-)^{T}\left(I-K_{k} H_{k}\right)^{T}+K_{k} v_{k} v_{k}^{T} K_{k}^{T}\right],
\end{aligned}
$$


however, the matrices are constant, therefore,

$$
\begin{aligned}
P_{k}(+)= & \left(I-K_{k} H_{k}\right) E\left[\tilde{x}_{k}(-) \tilde{x}_{k}(-)^{T}\right]\left(I-K_{k} H_{k}\right)^{T}+\left(I-K_{k} H_{k}\right) E\left[\tilde{x}_{k}(-) v_{k}^{T}\right] K_{k}^{T}+ \\
& K_{k} E\left[v_{k} \tilde{x}_{k}(-)^{T}\right]\left(I-K_{k} H_{k}\right)^{T}+K_{k} E\left[v_{k} v_{k}^{T}\right] K_{k}^{T},
\end{aligned}
$$

but, the definitions to be used are,

$$
\begin{array}{ll}
E\left[\tilde{x}_{k}(-) \tilde{x}_{k}(-)^{T}\right] & =P_{k}(-), \\
E\left[v_{k} v_{k}^{T}\right] & =R_{k}, \\
E\left[\tilde{x}_{k}(-) v_{k}^{T}\right] & =E\left[v_{k} \tilde{x}_{k}(-)^{T}\right]=0,
\end{array}
$$

so the posterior error covariance matrix becomes,

$$
P_{k}(+)=\left(I-K_{k} H_{k}\right) P_{k}(-)\left(I-K_{k} H_{k}\right)^{T}+K_{k} R_{k} K_{k}^{T} .
$$

The performance index, $J_{k}=E\left[\tilde{x}_{k}(+)^{T} \tilde{x}_{k}(+)\right]$ is defined and the Kalman gain $K_{k}$ can be determined to minimize this performance index with respect to $K_{k}$ as,

$$
\frac{\partial J_{k}}{\partial K_{k}}=-2\left(I-K_{k} H_{k}\right) P_{k}(-) H_{k}^{T}+2 K_{k} R_{k}=0 .
$$

then,

$$
K_{k}=P_{k}(-) H_{k}^{T}\left[H_{k} P_{k}(-) H_{k}^{T}+R_{k}\right]^{-1} .
$$

The discrete time Kalman filter algorithm is shown in Fig. 3.1 with the timing propagations and matrix updates, Gelb [58].

\subsubsection{Summary of Kalman Filter}

Fig. 3.2 illustrates the flow chart of the Kalman filter algorithm used. The initial conditions for the covariance matrix, $P_{k}(+)$, state, $\hat{x}_{k}(+)$, and $Q, R$ should be set. The step moves to "Time Update," which updates the state and the covariance matrix, as well as the measurement. The gain matrix is calculated according to equation (3.66). Next, the 


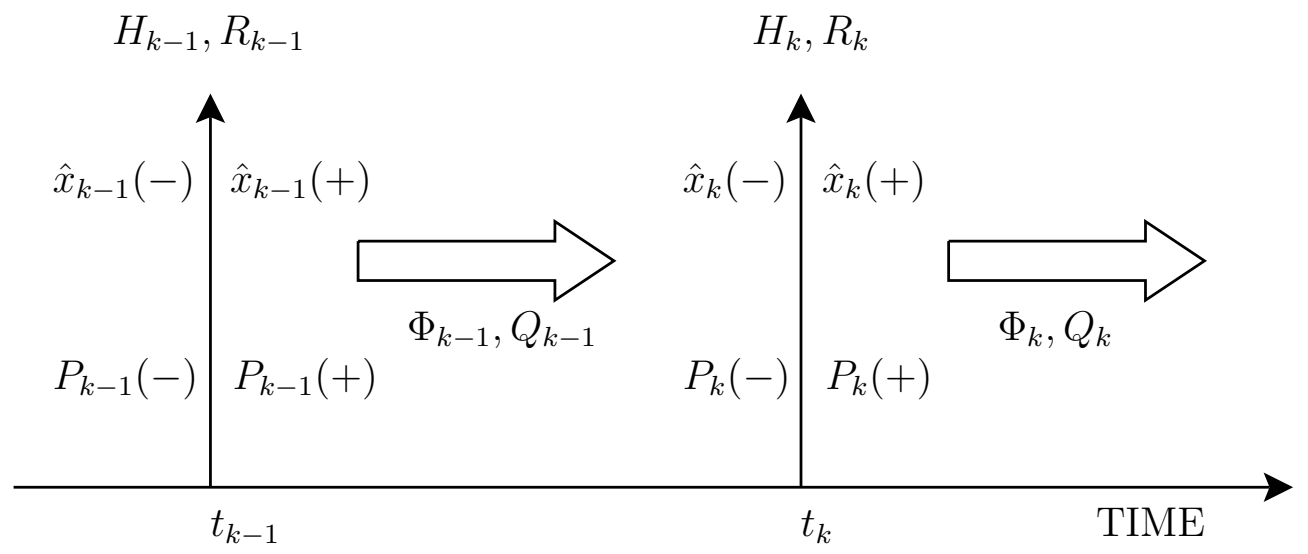

Figure 3.1: Discrete Kalman filter timing diagram.

estimated state needs to be corrected, using measurements, i.e. the pressure drop profiles in the "Correct" step. This is accomplished by increasing the time step and going back to "Time Update."

\subsection{Experimental Results}

The experiments are performed under two different sets of operating conditions. One set is the same operating conditions as in the built system model, shown in Table 3.2 while the other set of operating conditions are shown in Table 3.3. The bed materials, the amount of solids in the inventory, and the humidity are the same as those of the conditions which existed when system was built, but $U_{g}$ (i.e. the main aeration of the riser), the move air, and the period are different. For the first case, the experimental results are shown in Fig. 3.3 for a high solids circulation rate, and in Fig. 3.4 for a low solids circulation rate. In both case, the solid lines represent the measured values from the spiral while the dash lines show the estimated SCR from the Kalman filter with the SCR dynamic model. The estimated values converge to measured values in a few seconds. The overshoots occur due to the initial conditions in the SCR system model and the Kalman estimator. 


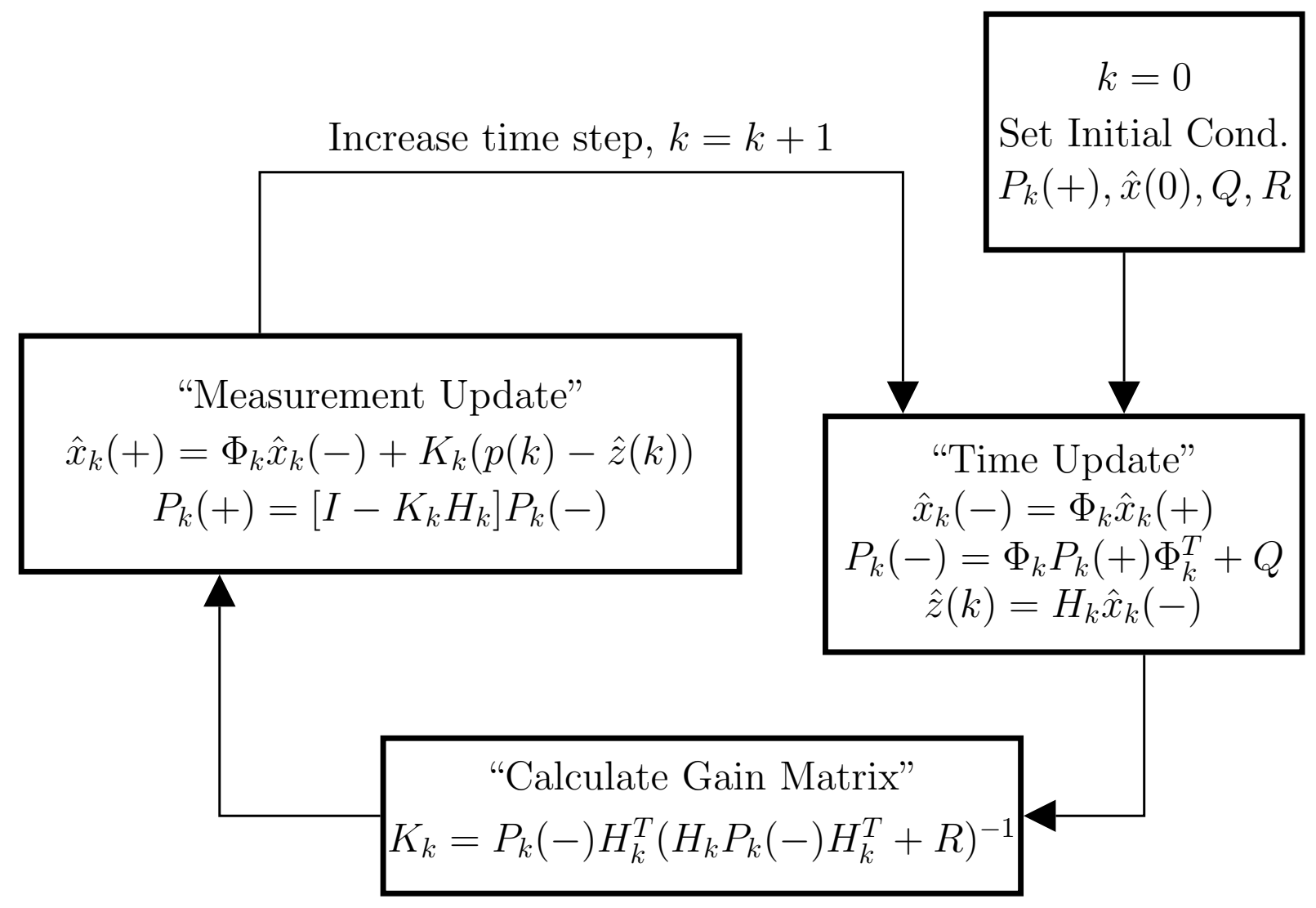

Figure 3.2: Flow chart of the Kalman filter algorithm with the linear system model.

\begin{tabular}{c||c|c||c}
\hline \hline Name Tag & \multicolumn{2}{|||}{ Properties } & Units \\
\hline Bed Material & Corks $\left(d=829 \mu, \rho_{s}=189\right)$ & $\mathrm{m}, \mathrm{kg} / \mathrm{m}^{3}$ \\
\hline$U_{g}$ & \multicolumn{2}{|c||}{4.05} & $\mathrm{~m} / \mathrm{sec}$ \\
\hline$F_{m}$ (Move Air) & 556 & 1214 & $\mathrm{SCFH}$ \\
\hline Periods & 120 & 90 & $\mathrm{sec}$ \\
\hline Inventory Solids & \multicolumn{2}{||}{68} & $\mathrm{~kg}$ \\
\hline Humidity & \multicolumn{2}{|c|}{90} \\
\hline \hline
\end{tabular}

Table 3.3: Operating conditions of the CFCFB to test the SCR system model. 


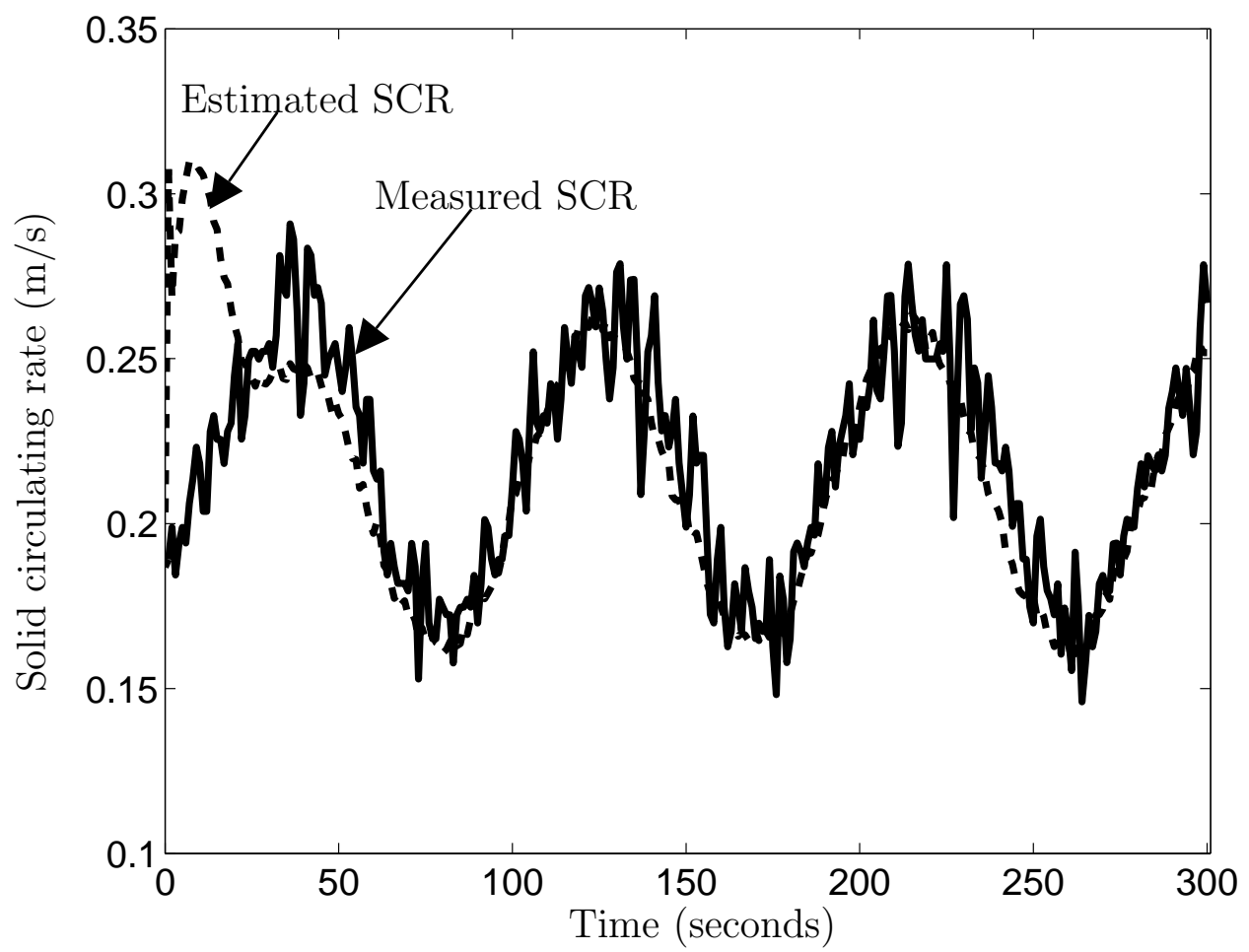

Figure 3.3: Experimental results for solids circulation rate in the case of a high rate.

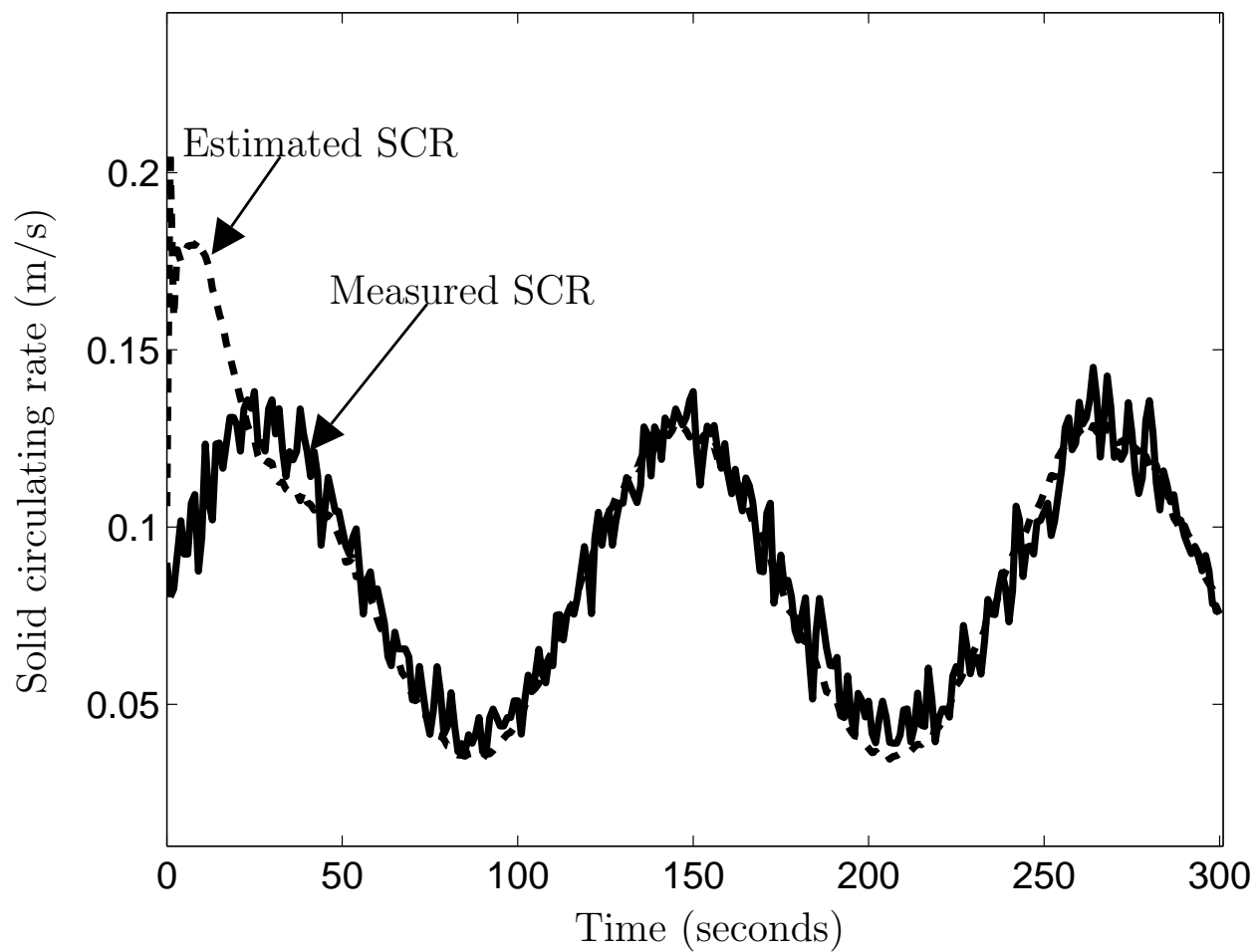

Figure 3.4: Experimental results for solids circulation rate in the case of a low rate. 
The experimental results for the other operating conditions are shown in Fig. 3.5 and Fig. 3.6 for both successful and unsuccessful estimations, respectively. When the previously built SCR system model was used, in one case the SCR is successfully estimated, however, in the other case the SCR is not successfully estimated even though the pressure drop profiles estimations are close to the measured ones. One of the pressure drop profiles in the case in which the SCR estimation is not working is shown in Fig. 3.7.

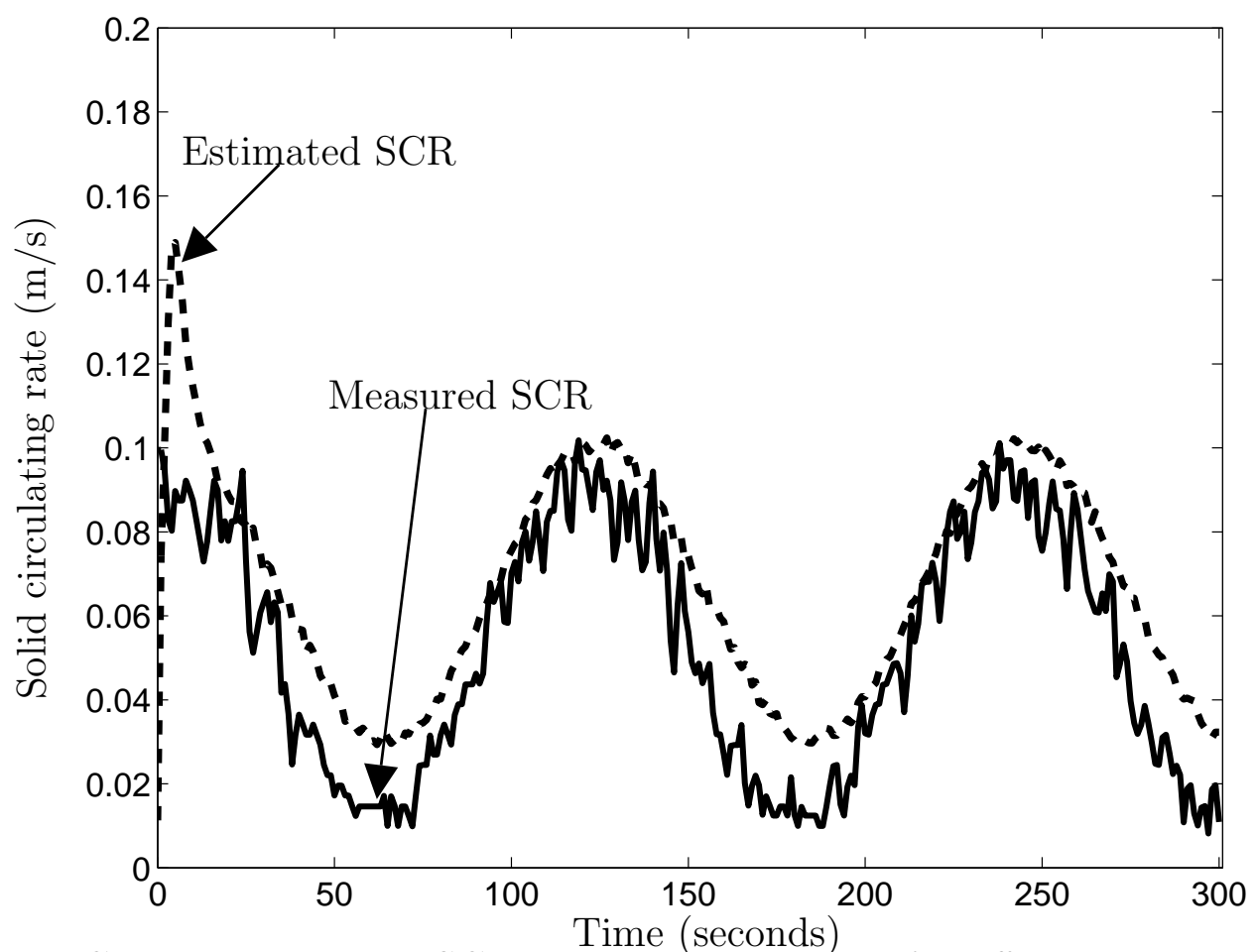

Figure 3.5: Correctly estimated SCR experimental results of a different operating condition.

\subsection{Bed-height Calculation}

The accurate knowledge of the bed-height in the standpipe of the CFCFB is one of the important factors in terms of controlling and improving the effective operation of the system. Knowing the bed-height is crucial in determining whether the system operates within the optimal regions or merely within operable regions. If the bed-height is too low 


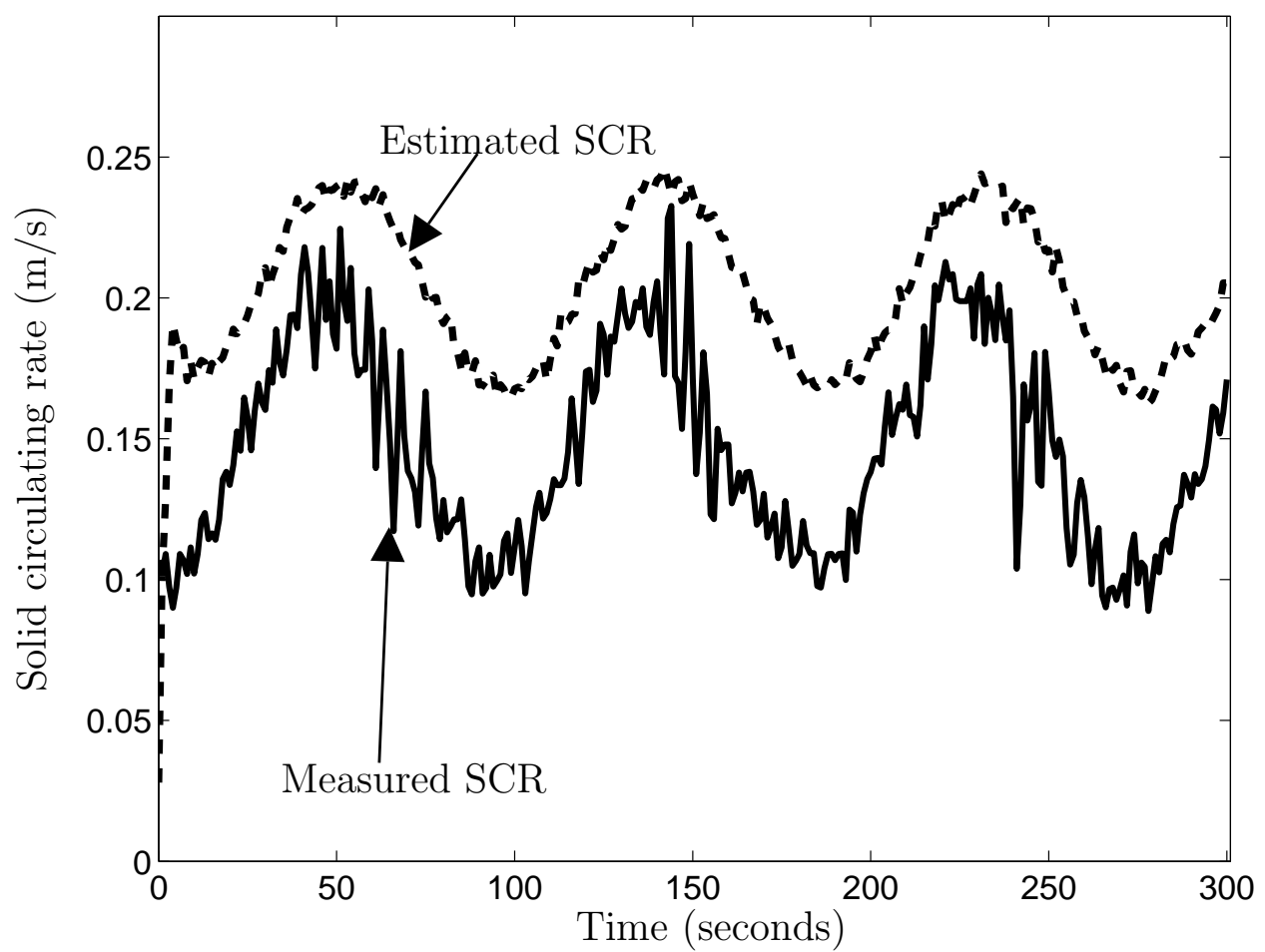

Figure 3.6: Not correctly SCR experimental results of a different operating condition.

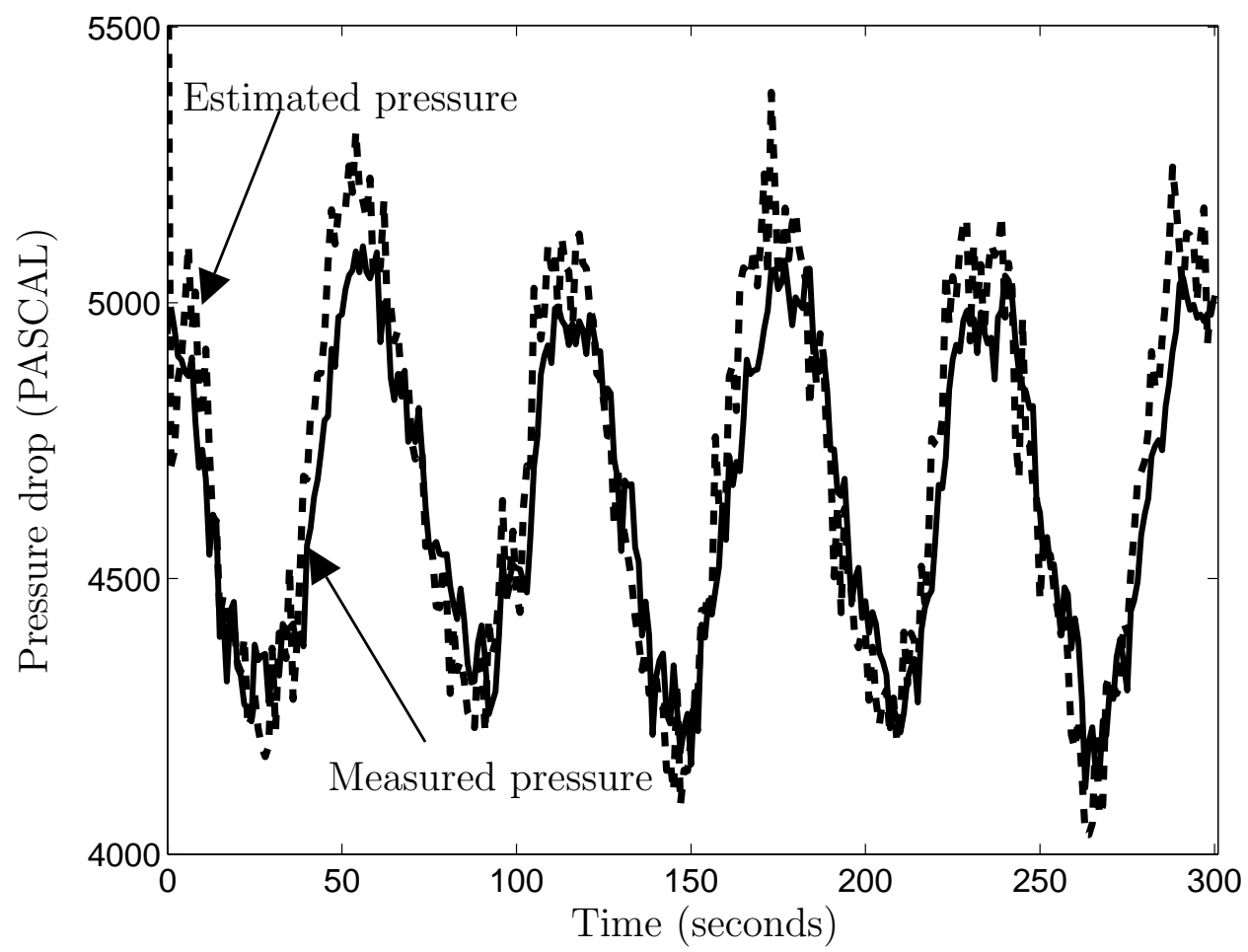

Figure 3.7: Pressure drop in case of not correctly estimated SCR. 


\section{CHAPTER III. SCR AND BED-HEIGHT ESTIMATES}

the standpipe pressure is too low, so the solid materials are not fluidized properly. The bedheight can be obtained from the estimation of the void fraction using filtering algorithms such as an extended Kalman filter Shim et al. [26], an $\mathcal{H}_{\infty}$ estimator Park et al. [11], or a sliding mode estimator. Here, simple and effective methods of finding the bed-height on the standpipe of the CFCFB, using three pressure drops are presented, Park et al. [12]. First, to explain the method of obtaining the bed-height from the pressure drops, an example plot of the pressure drop profile versus the bed-height is shown in Fig. 3.8. This figure illustrates the straight line across one point where the slope changes. There are two abrupt change points. The standpipe bed-height lies somewhere in-between these two points. This third point divides the standpipe into two regions, lean and dense beds, defined as the bed-height of the standpipe in the CFCFB. The $\Delta p_{l}$ is the pressure drop in the lean phase of the standpipe, while the $\Delta p_{d}$ is the pressure drop of the dense bed. The goal of this research is to find this dividing point in order to identify the bed-height based on these pressure drops.

In this research, the basic equation relating the pressure drop to the void fraction, Park et al. [12] with the two-region separated by bed-height is used in order to obtain the bed-height as,

$$
\Delta P=\rho_{s}(1-\varepsilon) g h,
$$

where $\rho_{s}$ is the density of solids, $\varepsilon$ is the void fraction, $g$ is the gravitational acceleration, and $h$ is the height of where the pressure drops are measured. Using the proportional relations between pressure taps and (3.67), the bed-height can be identified. The void fraction on the very top is assumed to be a constant or obtained from the pressure drop while the bed-height is less than $8.2 \mathrm{~m}$ at the point where the last pressure tap is located. In some cases, these bed-height calculation methods cannot be used because the bed-height lies between the top two pressure taps. Therefore, a modified bed-height calculation method is introduced. 


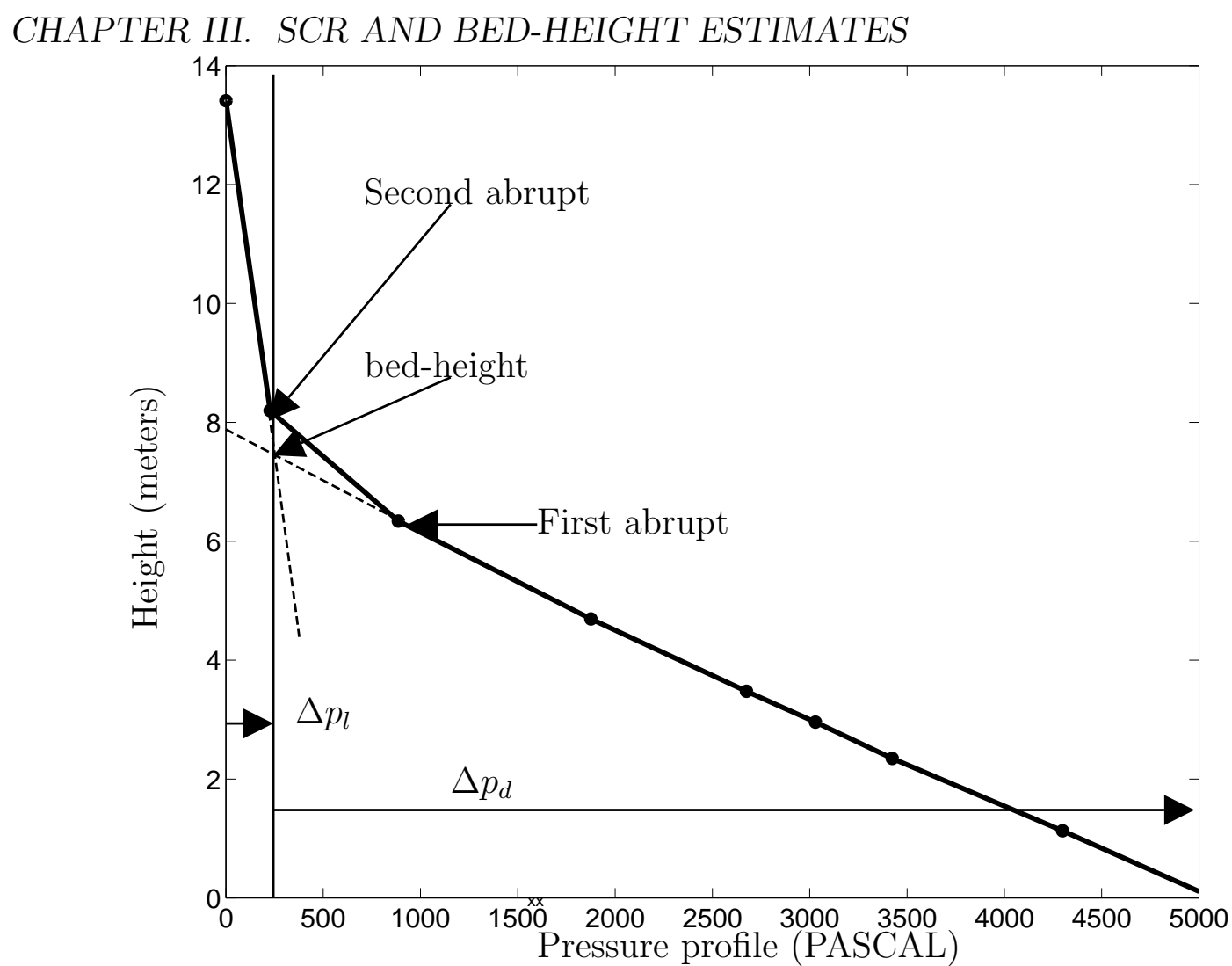

Figure 3.8: Sample plot of pressure profile vs. heights.

\subsubsection{Calculating Bed-height of Less Than 8.2 Meters}

The standpipe can be modeled as two homogeneous sections, dense and lean phases, with respect to the bed-height as shown in Fig. 3.9. The upper part is the lean region where the density of solids is low. The lower part is the dense region where the density of solids is high. In the dense region, the pressure drop is higher than in the lean region. Let subscripts $L, D$, and $T$ represent the lean phase, the dense phase, and the total phase, respectively. Achieving pressure balance produces the equation for the lean region,

$$
\Delta P_{1}=\frac{z_{T}-z_{2}}{z_{L}} \Delta P_{L}
$$

while the equation for the dense region is,

$$
\Delta P_{2}=\frac{z_{2}}{z_{D}} \Delta P_{D}
$$




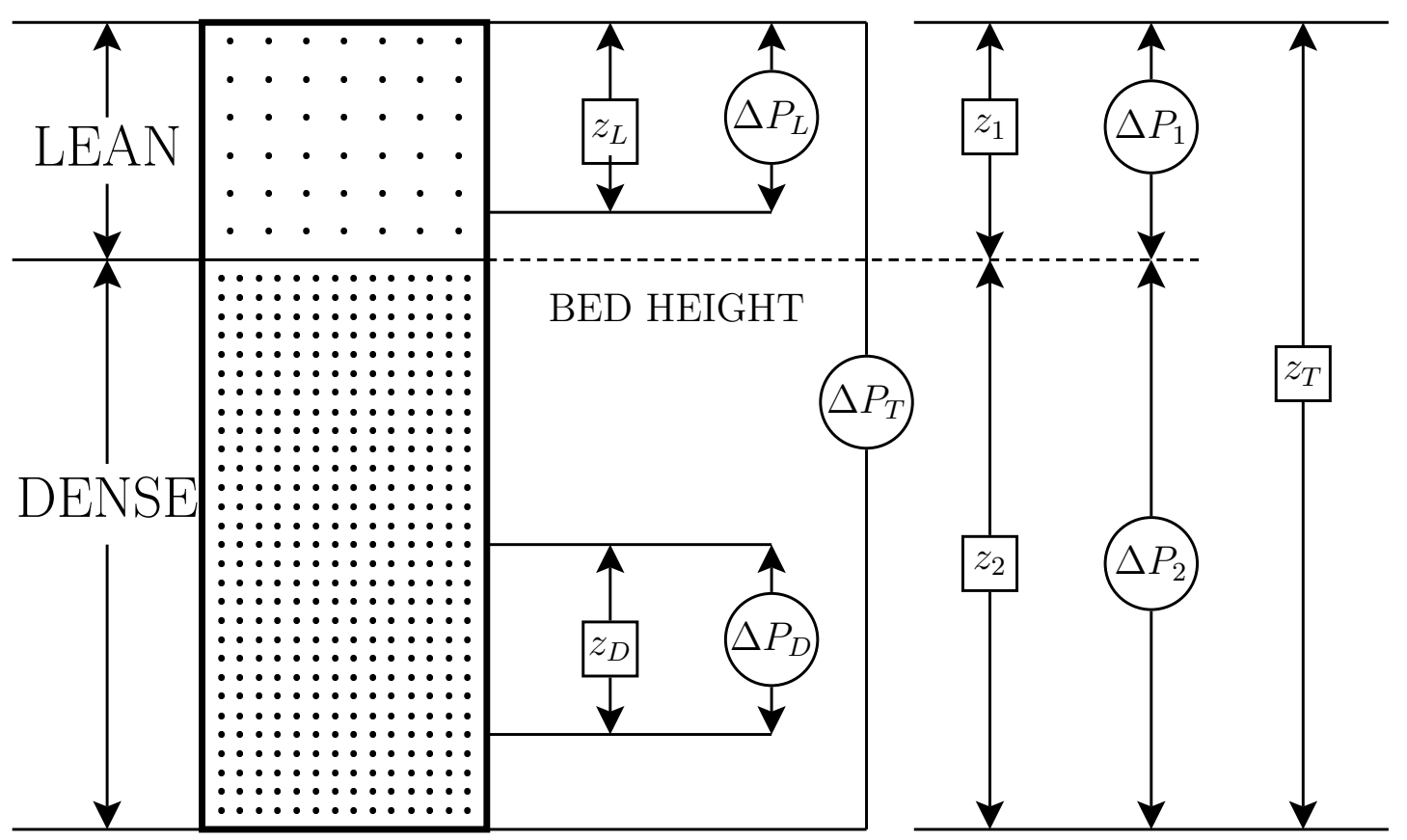

STANDPIPE

Figure 3.9: Simple homogeneous 2-region model of the standpipe.

where $\Delta P_{1}$ is the overall pressure drop for the lean phase, $\Delta P_{2}$ is the overall pressure drop for the dense phase, $z_{2}$ is the bed-height that separates the bed into dense and lean phases, $z_{L}$ is the height difference where the pressure drop is measured for the lean phase, $z_{D}$ is the height difference where the pressure drop is measured for the dense phase, and $z_{T}$ is the total standpipe height. The total pressure drop across the standpipe is given by,

$$
\Delta P_{T}=\Delta P_{1}+\Delta P_{2}
$$

taking (3.68) and (3.69) into (3.70), the overall pressure drop in the standpipe is,

$$
\Delta P_{T}=\frac{\Delta P_{L}}{z_{L}}\left(z_{T}-z_{2}\right)+\frac{\Delta P_{D}}{z_{D}} z_{2} .
$$

Rearranging the above equation, the bed-height equation can be found to be,

$$
z_{2}=\frac{\Delta P_{T}-\frac{\Delta P_{L}}{z_{L}} z_{T}}{\frac{\Delta P_{D}}{z_{D}}-\frac{\Delta P_{L}}{z_{L}}} .
$$




\subsubsection{Modified Bed-height Calculation Method}

In this case, the bed-height calculation procedure can not be applied in order to obtain the bed-height because the bed-height lies between the top two pressure taps. Hence, another algorithm must be developed to calculate the bed-height. A detailed diagram of these concepts is shown in Fig. 3.10. Since the length of the top pressure measurement is known, the length of the lean region can be defined as,

$$
h_{l} \equiv z_{L}-h
$$

where $h_{l}$ is the length of the lean region in the very top pressure drop measurement, $z_{L}$ is the length of the very top pressure drop measurement, and $h$ is the dense region in the top pressure drop measurement. As seen in Fig. 3.10, the bed-height can be calculated by $8.2+h$ when $h$ is known. Defining the pressure drop for the lean phase to be $\Delta P_{l}$, and the pressure drop for the dense phase greater than the height of 8.2 meters to be $\Delta P_{d}$, the pressure equation in the section that includes the bed-height is satisfied as,

$$
\Delta P_{L}=\Delta P_{l}+\Delta P_{d}
$$

Now, using the definition of pressure drop from the void fraction in (3.67) yields,

$$
\rho_{s}\left(1-\varepsilon_{L}\right) g z_{L}=\rho_{s}\left(1-\varepsilon_{l}\right) g\left(z_{L}-h\right)+\rho_{s}\left(1-\varepsilon_{d}\right) g h,
$$

where, $\varepsilon_{L}$ is the void fraction of the top pressure measurement section, $\varepsilon_{l}$ is the void fraction in the lean bed, and $\varepsilon_{d}$ is the void fraction in the dense bed. Rearranging (3.75), the bed-height becomes,

$$
h=\frac{\left(\varepsilon_{l}-\varepsilon\right) z_{T}}{\varepsilon_{l}-\varepsilon_{d}} .
$$

The lean phase void fraction, $\varepsilon_{l}$, is calculated from the previous pressure drop when the bed-height is less than 8.2 meters or can be assumed to be a constant value. The dense pressure drop from the bottom of the standpipe to 8.2 meters in the standpipe can be used to calculate the dense void fraction, $\varepsilon_{d}$. 


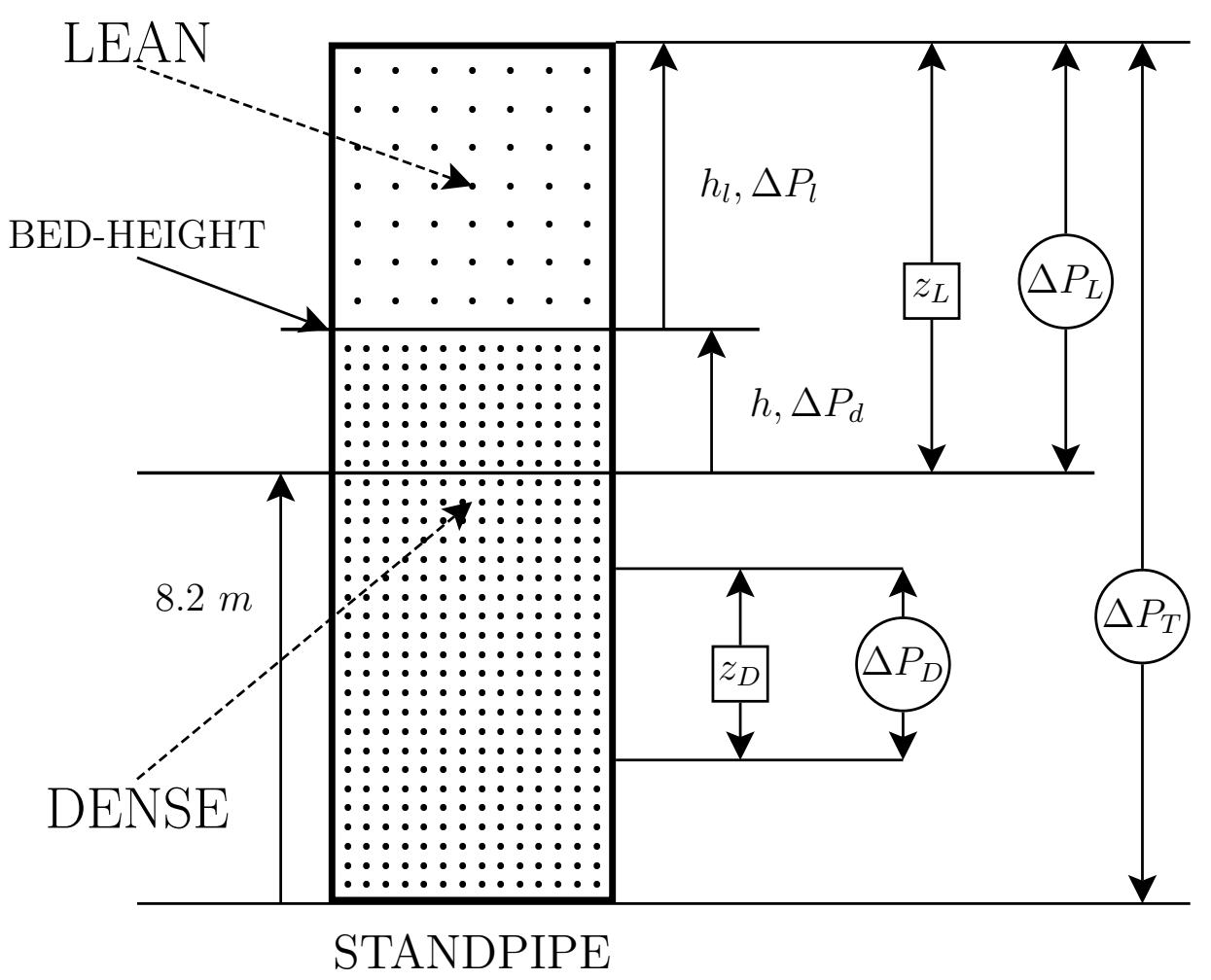

Figure 3.10: Diagram for the modified 2-region model.

\subsubsection{Test Results}

The test simulation is taken from data collected on December 06, 2001. There are several cases, but here the selected cases are illustrated to show the effectiveness of these 2-region and modified 2-region models. There are two figures, Figs 3.11 and 3.12 for each 2-region model and modified 2-region model, respectively. In the figures, the solid line represents the experimental measurement while the dash line represents the calculation results. The experimental results have a 15 second sampling time; however, the calculation results have a one second sampling time. The bed-height measurements are not currently available automatically from any sensor, so human data recording is utilized. The pressure drop measurements, however, can be recorded by the data acquisition system, so the 


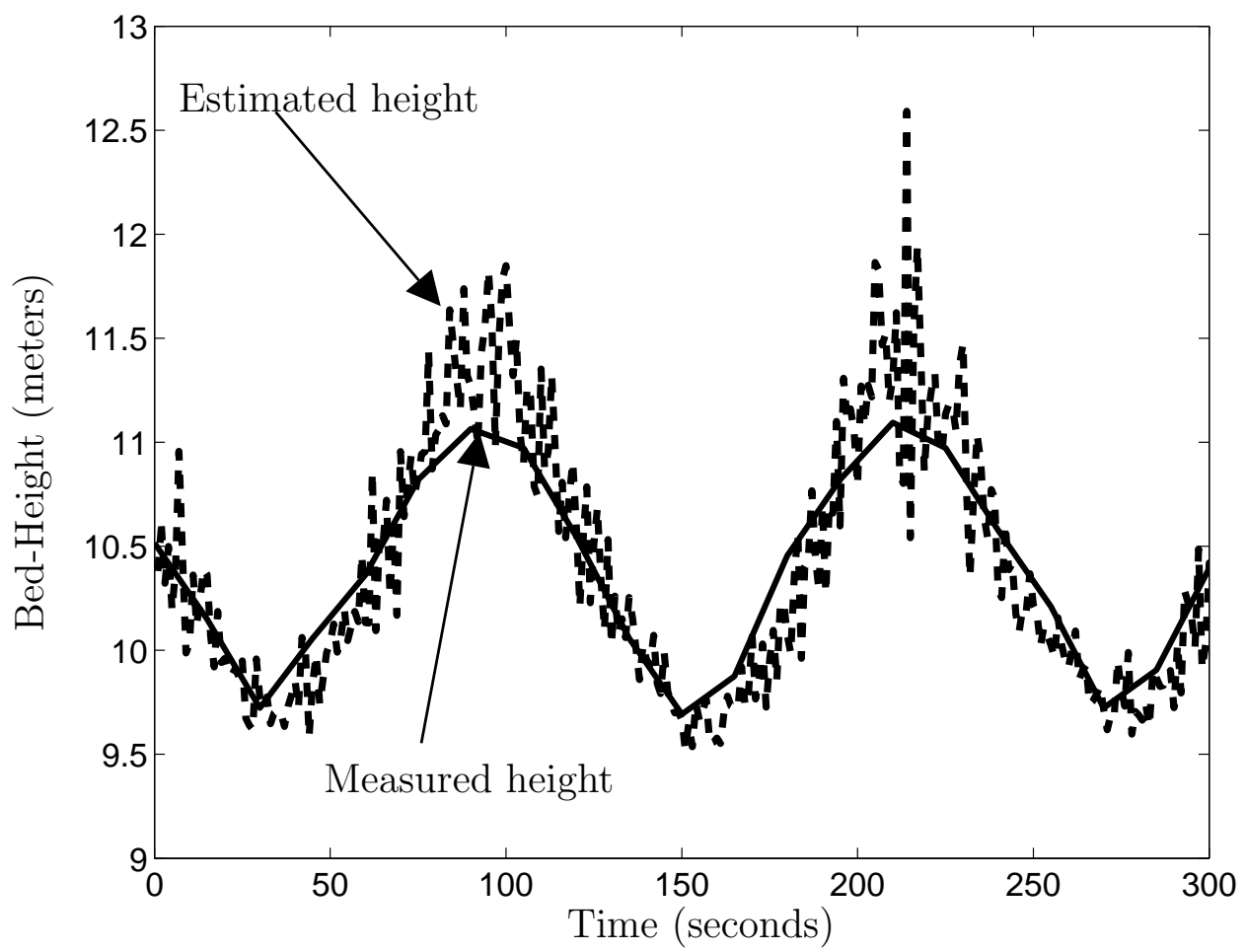

Figure 3.11: Experimental results for the 2-region model.

estimated bed-heights are available for every second.

\subsection{Summary}

In this Chapter, the solids circulation rate estimation and the bed-height calculation were developed and discussed. The linear discrete dynamic system was developed in order to estimate the SCR, applying the Kalman filter using the pressure drops along the standpipe. The least squares estimation and the extended observability matrix formed from state space model were utilized through the subspace algorithm. Once the dynamic model had been built, the system dynamic was transported to a different form without the SCR in the output vector. After the system model was obtained, the Kalman filter was utilized to estimate the solids circulation rate using measured pressure drops in the standpipe and the riser as well as the move air. The experimental results show that this 


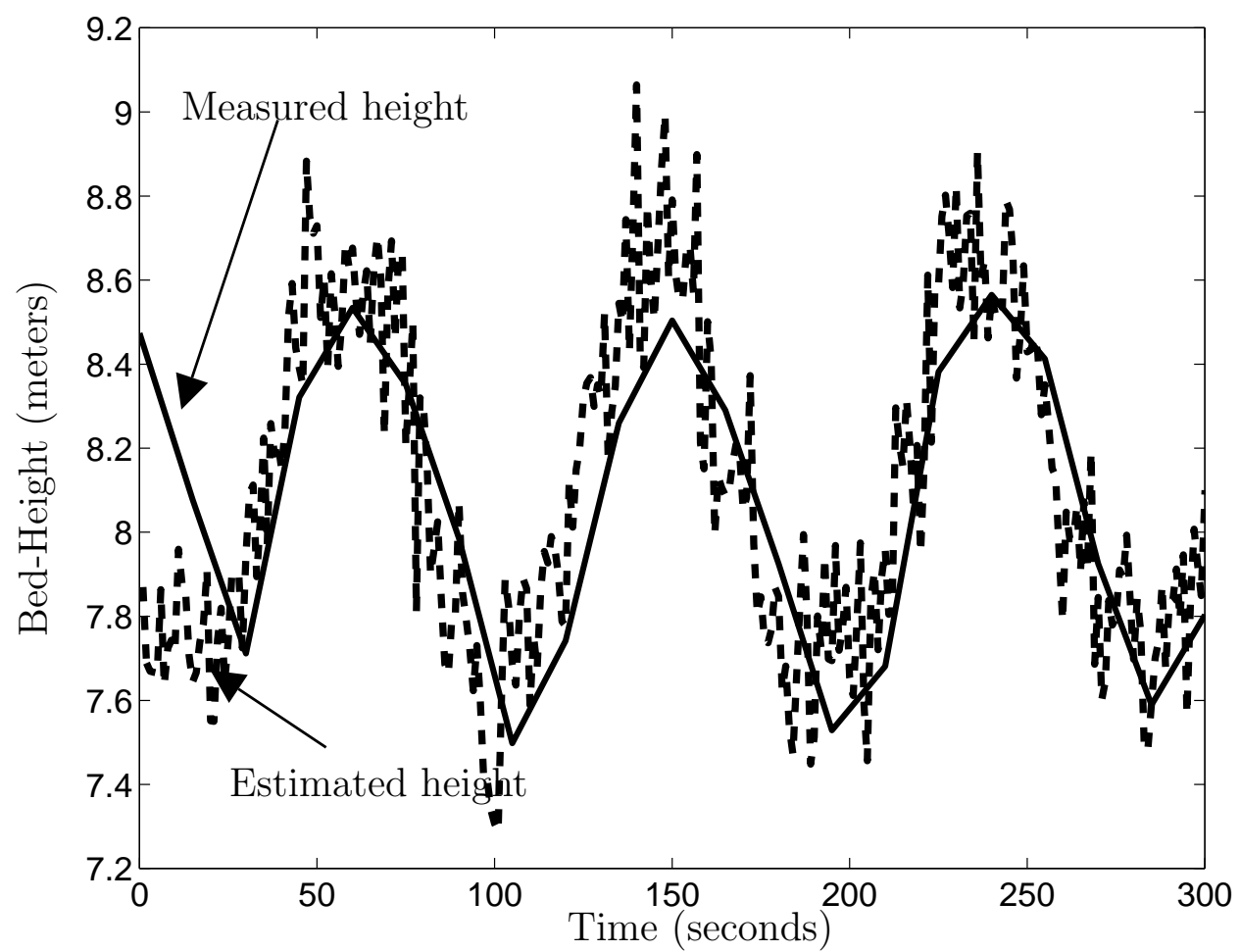

Figure 3.12: Experimental results for the modified 2-region model.

method estimates the SCR successfully in a given operating condition. To calculate the bed-height, the 2-region method was utilized using three pressure drops. The standpipe was modeled in two homogeneous regions, i.e. dense and lean, which were separated by the bed-height. The modified 2-region method was used if the bed-height was higher than 8.2 meters since there was no pressure tap. The test results show that these methods are effective and reliable. 


\section{Chapter IV}

\section{Sliding Mode Estimation Technique}

\subsection{Introduction}

The sliding mode observer technique is based on the Lyapunov stability criteria and can be applied to a non-linear system because of its stability and robustness, Xiong and Saif [59], Islam et al. [60]. To explain better the sliding mode observer, the sliding mode control technique is first discussed. This technique can transfer the complicated non-linear system controller into a simple $1^{\text {st }}$ or $2^{\text {nd }}$ order system controller, using a first or second order switching surface, Park et al. [48], Jacque et al. [61]. The Lyapunov stability criteria demonstrate that if the energy function can be found as a positive definite and a derivative of its function exists, the system is stable, as long as the derivative of the energy function is negative, i.e.

$$
\frac{\mathrm{d} V(\vec{x}, t)}{\mathrm{d} t}<0
$$

where $V(\vec{x}, t)$ is the energy function, related to the system or controller dynamic, and $\vec{x}$ is the system state vector. The sliding mode estimator uses the same idea as does the controller. The switching surface that satisfies the Lyapunov stability is first found, then the gain that drives the system along the switching surface is found according to 


\section{CHAPTER IV. SLIDING MODE ESTIMATION TECHNIQUE}

the Lyapunov stability criteria. Once these criteria are met, the estimator guarantees the stability and the convergence to the switching surface. The practical applications of the sliding mode estimation to the induction machine are applied in Yan et al. [35], Utkin [36], Benchaib et al. [37], Derdiyok et al. [38]; the MEMs electrostatic and load torque estimations applications are given in Park et al. [48]. In Section 4.2, the variable structure system and controller example, which are essential to understanding the sliding mode estimator, are given. In Section 4.3, the method of applying the sliding mode estimator to the CFCFB is discussed.

\subsection{Variable Structure System}

\subsubsection{The Phase Plane}

First, the phase plane plots need to be considered in order to understand the variable structure system (VSS) or the sliding mode controller, Jacque et al. [61]. In order to visualize the phase plane, the second order differential equation is considered,

$$
\ddot{x}+a_{1} \dot{x}+a_{2} x=0,
$$

where $\dot{x}$ represents the time derivative of $x$, while $a_{1}$ and $a_{2}$ are constants. If this equation is solved, its roots can be complex or real depending on the constants $a_{1}$ and $a_{2}$ as,

$$
x=\alpha+j \beta,
$$

where $\alpha$ and $\beta$ are real numbers and $j$ is $\sqrt{-1}$. Depending on these values, several phase plane portraits exist as shown in Figs. 4.1 to 4.6. The first case is the stable node shown in Fig. 4.1. The left side of Fig. 4.1 shows the location of the eigenvalues in the complex plane while the right side shows the trajectories of the system in the phase plane. Because both eigenvalues are located on the left-hand side of real axis, the system trajectories are 
stable, Nise [62]. Whenever the initial conditions start, the system is driven to the origin by itself. The second case is the unstable node, as shown in Fig. 4.2. The trajectories of the phase plane are unstable since both eigenvalues are located on the right-hand side of the real axis.

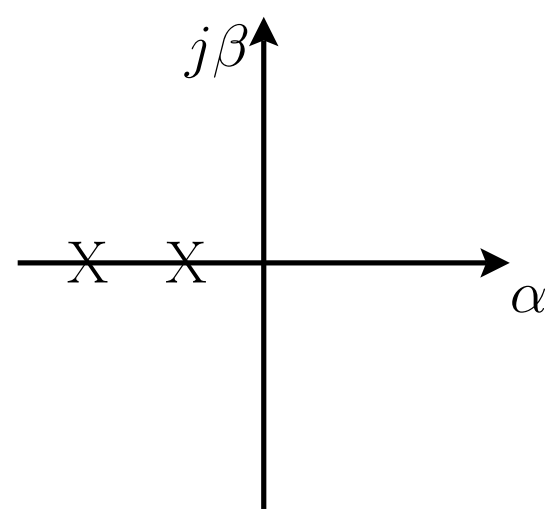

The Complex Plane

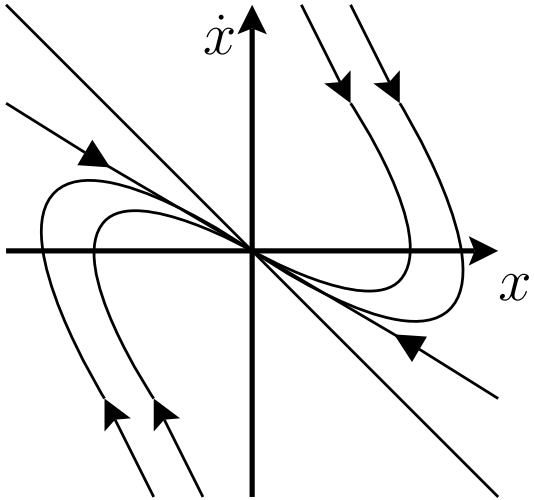

The Phase Plane

Figure 4.1: Stable node of the phase plane.

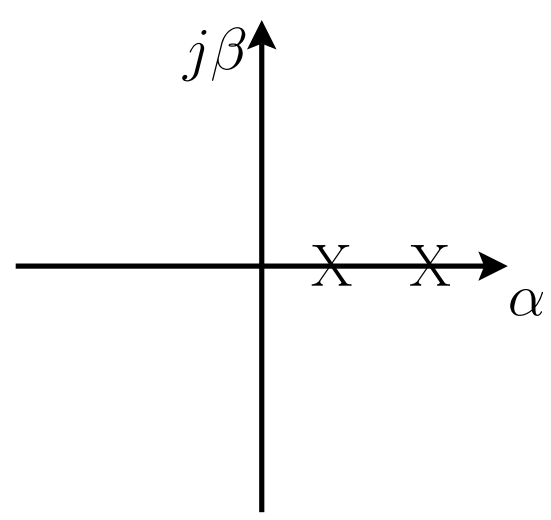

The Complex Plane

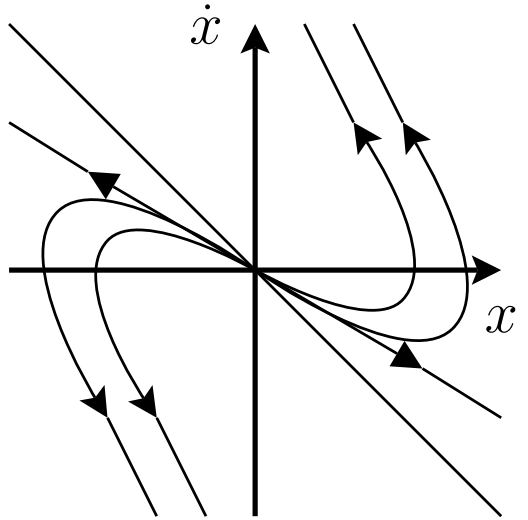

The Phase Plane

Figure 4.2: Unstable node of the phase plane.

In this case, the system is unstable whenever the initial conditions start, unless a control action is taken. The other two important cases of the sliding mode controller are the saddle point and the center point, as shown in Figs. 4.3 and 4.4, respectively. For the saddle point, 


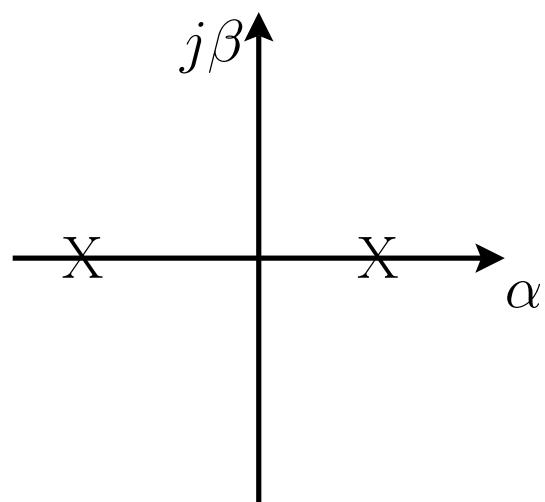

The Complex Plane

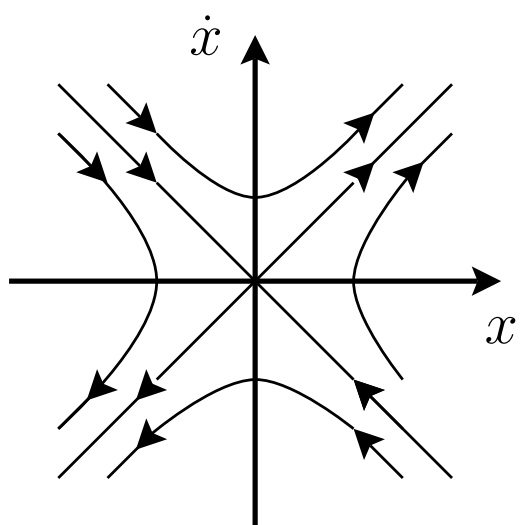

The Phase Plane

Figure 4.3: Saddle point of the phase plane.

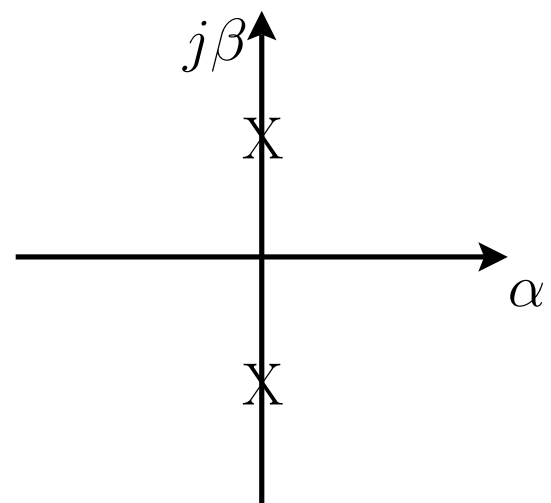

The Complex Plane

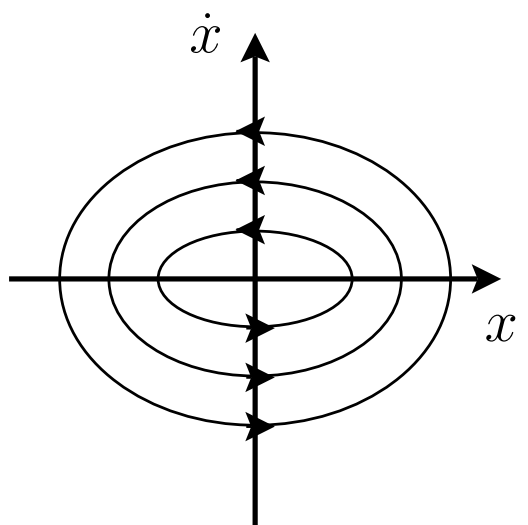

The Phase Plane

Figure 4.4: Center point of the phase plane.

one of the eigenvalues is located on the right-hand side of the real axis while the other is located on the left, in such a way that the trajectories of the phase plane look like saddles. The system stabilities depend on the initial conditions. The system can either go to the origin or be blown out to infinity. On the other hand, at the center point the eigenvalues are located on the imaginary axis (one on the top and the other on the bottom) in such a way that the trajectories of the phase plane look like ellipse curves. In this case, the trajectories of the phase plane can not go either to the origin nor to infinity. They simply circulate around the origin along the ellipse curves. 
The last two cases are a stable focus and an unstable focus depending on the locations of the eigenvalues, as shown in Figs. 4.5 and 4.6. Like the stable node, the stable focus is stable and converges to the origin by itself, no matter what the initial starting conditions are. In the case of the unstable focus, the system trajectories blow out to infinity wherever the initial conditions start.

\subsubsection{Basic Concept of the Sliding Mode Control}

To explain the sliding mode control technique, a block diagram of the second order system is shown in Fig. 4.7. The plant could be an unstable non-linear system, Jacque et al. [61]. Depending on the variable $a$, the system could be either the center point or the saddle point, which were discussed in the previous section.

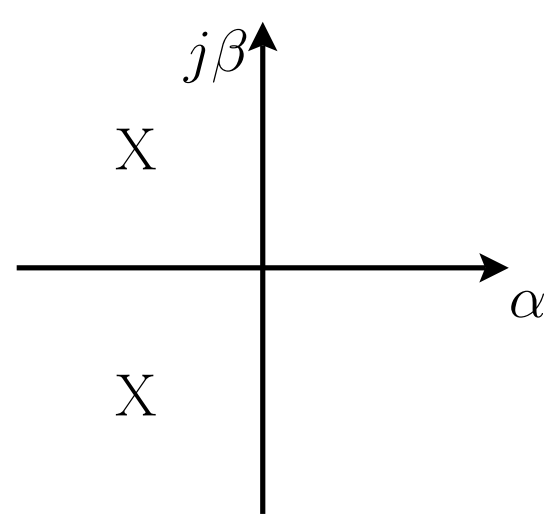

The Complex Plane

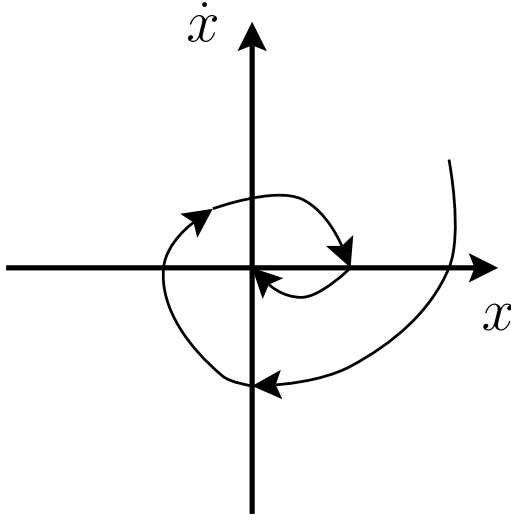

The Phase Plane

Figure 4.5: Stable focus of the phase plane.

Assuming the gain, $a$ could be adjustable, then the system can be controlled to the center point or the saddle point by changing the sign of the gain, $a$. If $a>0$, the system is the center point while if $a<0$, the system is the saddle point. The system dynamic 


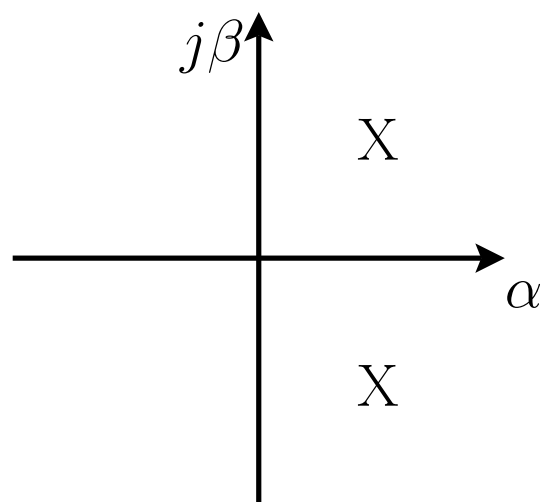

The Complex Plane

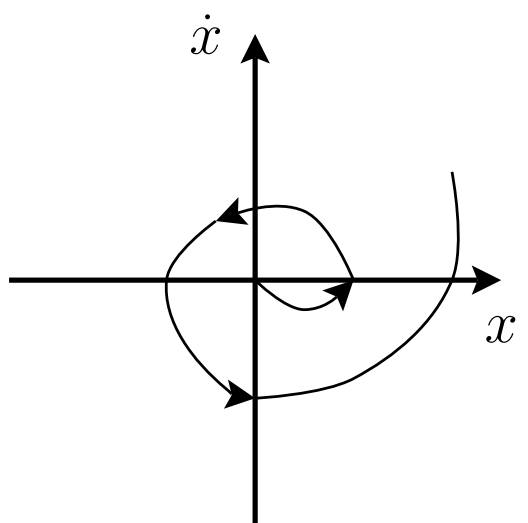

The Phase Plane

Figure 4.6: Unstable focus of the phase plane.

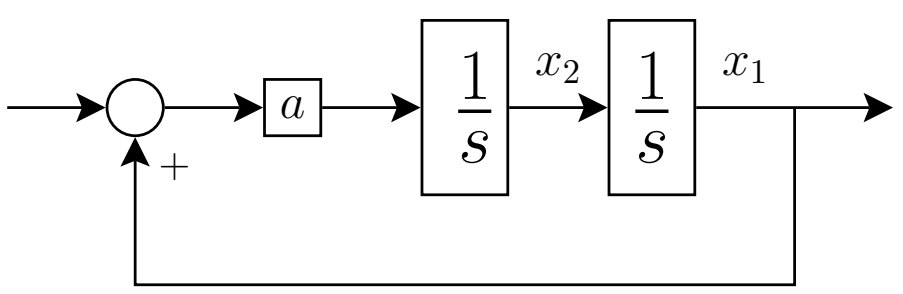

Figure 4.7: Second order system for sliding mode controls

equation of the second order system is given as,

$$
\begin{aligned}
& \dot{x}_{1}=x_{2}, \\
& \dot{x}_{2}=a x_{1} .
\end{aligned}
$$

If these two cases (i.e. parabola and ellipse) are combined, the control sequence of the sliding mode controller can be found. The basic concept of the sliding mode controller is shown in Fig. 4.8. The system starts at an initial operating point, then continually employs the control strategy (i.e. changing the gain sign) until the system reaches the origin. If the trajectory reaches a parabola $(a<0)$, the sign of gain $a$ is changed to positive. When the system trajectory reaches an ellipse, the gain is changed to negative to follow this trajectory. The control process is repeated until the system reaches the origin. Utilizing this simple but effective method takes too long to drive the system to the origin, 


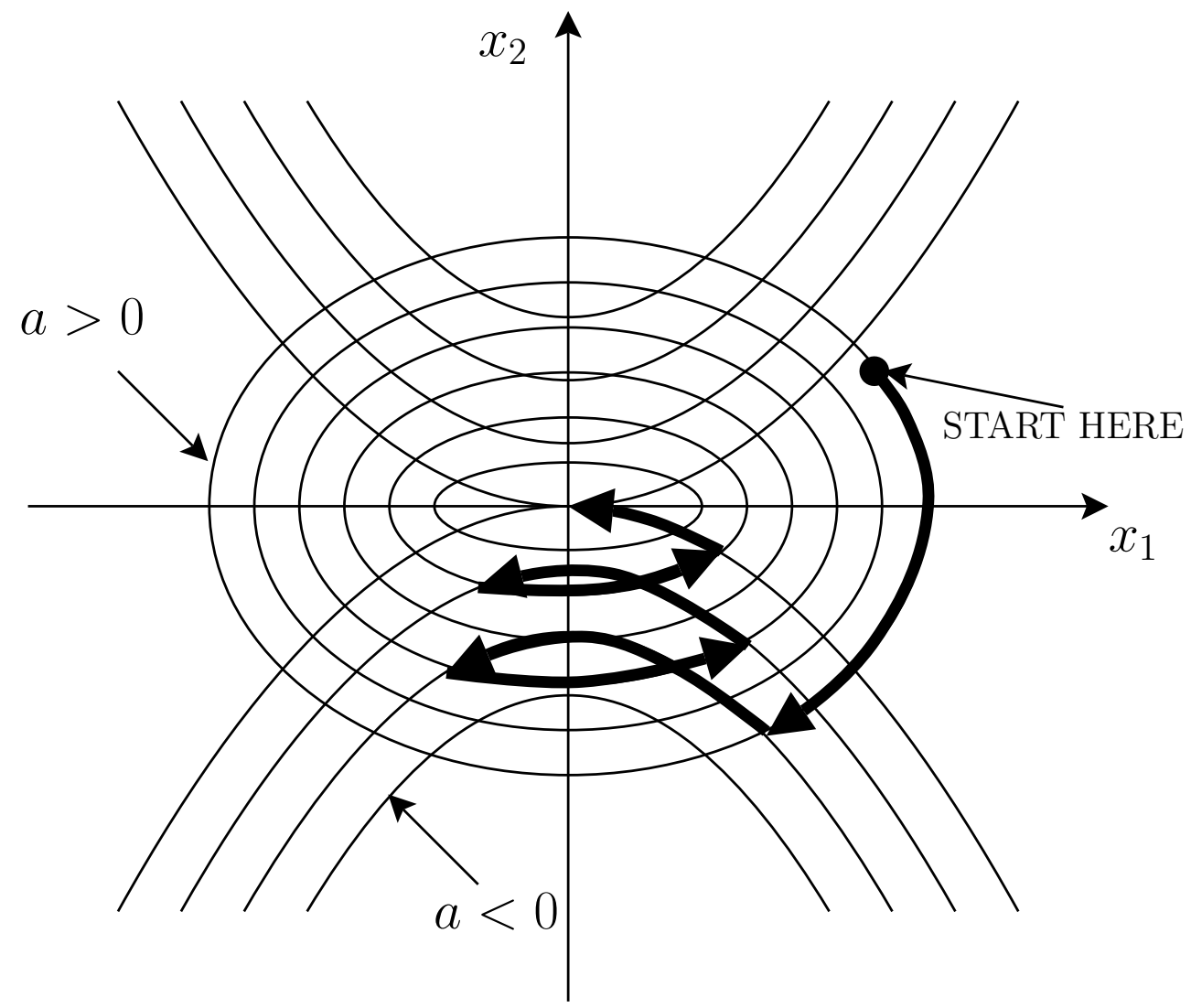

Figure 4.8: Basic concept of a sliding mode control.

and the frequency of time changes of the sign of the gain $a$ is unknown. The concept of using the switching line and driving the system quickly to the origin is introduced and shown in Fig. 4.9. As in the basic idea of the sliding mode controller, the concept starts with an initial operating point and waits until the trajectory reaches the switching surface. Thereafter, the gain or the control strategies can be found, based on the Lyapunov stability criteria. The properties of the sliding mode controller depend on the slope of the sliding surface. If the slope of the sliding surface is greater than one and increasing, the controller drives the system to the origin faster, but the controller might have the chattering problem and sometimes becomes unstable. If the slope of the sliding surface is less than one and decreasing, the controller drives the system to the origin slower, but the controller is rather 


\section{CHAPTER IV. SLIDING MODE ESTIMATION TECHNIQUE}

stable. As determined by the switching surface based on the state variables, the energy

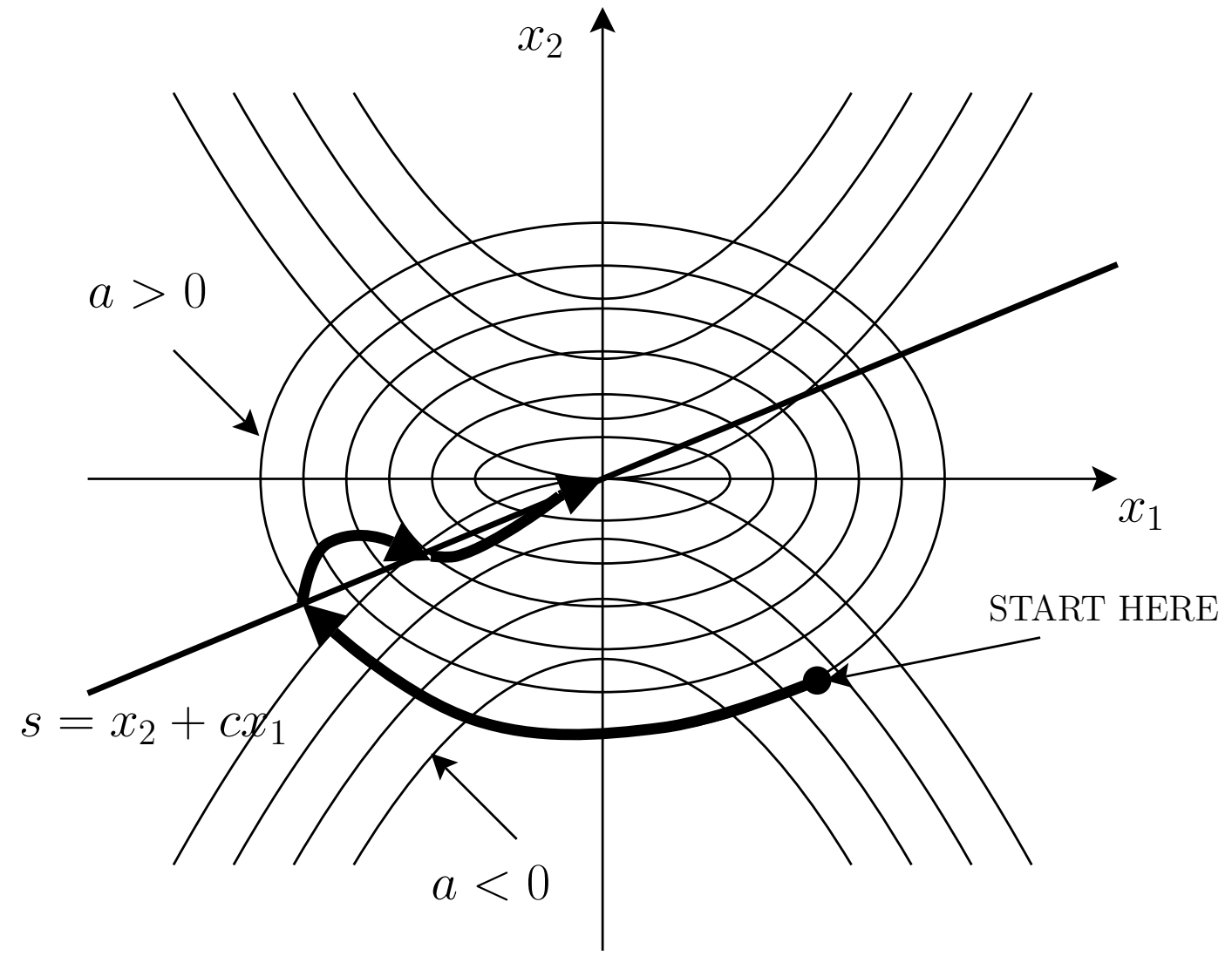

Figure 4.9: Basic concept of a sliding mode control using a switching line.

function usually is defined using the error dynamic as,

$$
V(\vec{x}, t)=\frac{1}{2} \mathbf{e}(\vec{x}, t) \mathbf{e}^{T}(\vec{x}, t),
$$

where $\mathbf{e}=\mathbf{y}(t)-\hat{\mathbf{y}}(t)$ is the error function, $\mathbf{y}(t)$ is the measurement vector, $\hat{\mathbf{y}}(t)$ is the estimated output vector, and $\vec{x}$ is the state vector. The system trajectories will converge to the switching surface as long as the Lyapunov stability criterion is met. 


\subsubsection{Example of a Sliding Mode Control}

A simple second order system example and the design procedure of the sliding controller are given in this section. First, the system equation is defined as,

$$
\begin{aligned}
& \dot{x}_{1}=x_{2}, \\
& \dot{x}_{2}=-a_{2} x_{2}-a_{1} x_{1}-b u
\end{aligned}
$$

where $a_{1}, a_{2}$, and $b$ are constant. The VSS control law can be found as the full state feed back,

$$
u=k_{1} x_{1}+k_{2} x_{2}
$$

where $k_{1}$ and $k_{2}$ are constants to be determined based on sliding mode control strategies. Let the sliding switching line be,

$$
s=\sigma=x_{2}+c x_{1}
$$

where $c$ is an arbitrary positive constant. From the stability condition, $V(t)>0$ and $\dot{V}(t)<0$ where $V(t)=\frac{1}{2} s s$ is an energy function for this system, the gains, $k_{1}$ and $k_{2}$ can now be determined. Obviously, the energy function is positive because $V(t)$ is squared as long as the switching line is real. Applying the derivative to the switching line and replacing the state derivatives with the system equation yields,

$$
\dot{s}=\dot{x}_{2}+c \dot{x}_{1}=-\left(a_{2}+b k_{2}-c\right) x_{2}-\left(a_{1}+b k_{1}\right) x_{1},
$$

multiplying the switching line, $s$ to the above equation, the derivative of the energy function becomes,

$$
\dot{V}(t)=s \dot{s}=-\left(a_{2}+b k_{2}-c\right) s x_{2}-\left(a_{1}+b k_{1}\right) s x_{1} .
$$

The derivative of the energy function should be negative to satisfy the Lyapunov stability criteria. So, the gains $k_{1}$ and $k_{2}$ are determined as,

$$
k_{1}=\left\{\begin{array}{ccc}
>-\frac{a_{1}}{b} & \text { where } & s x_{1}>0 \\
<-\frac{a_{1}}{b} & \text { where } & s x_{1}<0
\end{array} \text { or } k_{1}=\left(-\frac{a_{1}}{b}+d_{1}\right) \operatorname{sgn}\left(s x_{1}\right),\right.
$$




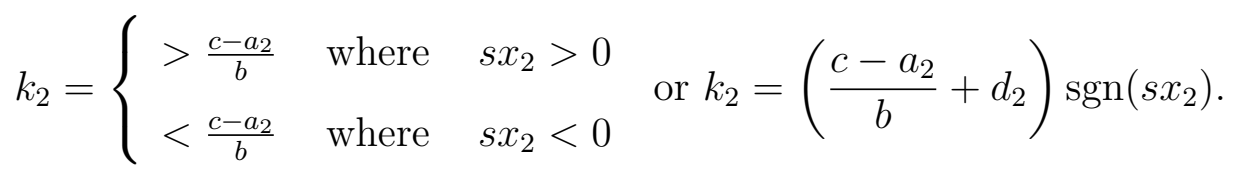

The simulation results for when $a_{1}=a_{2}=1.0, b=0.5$, and $c=3.0$ are shown in Fig. 4.10 for the speed and position and in Fig. 4.11 for the phase plane plot. This system is stable, so the position and the speed converge to the origin without the controller. However, with a sliding mode controller the system trajectories converge to the origin faster and without fluctuations. The phase plane plot shows that a plot with a controller converges to the origin in a straight line instead of spiraling while one without the controller spirals to the origin.
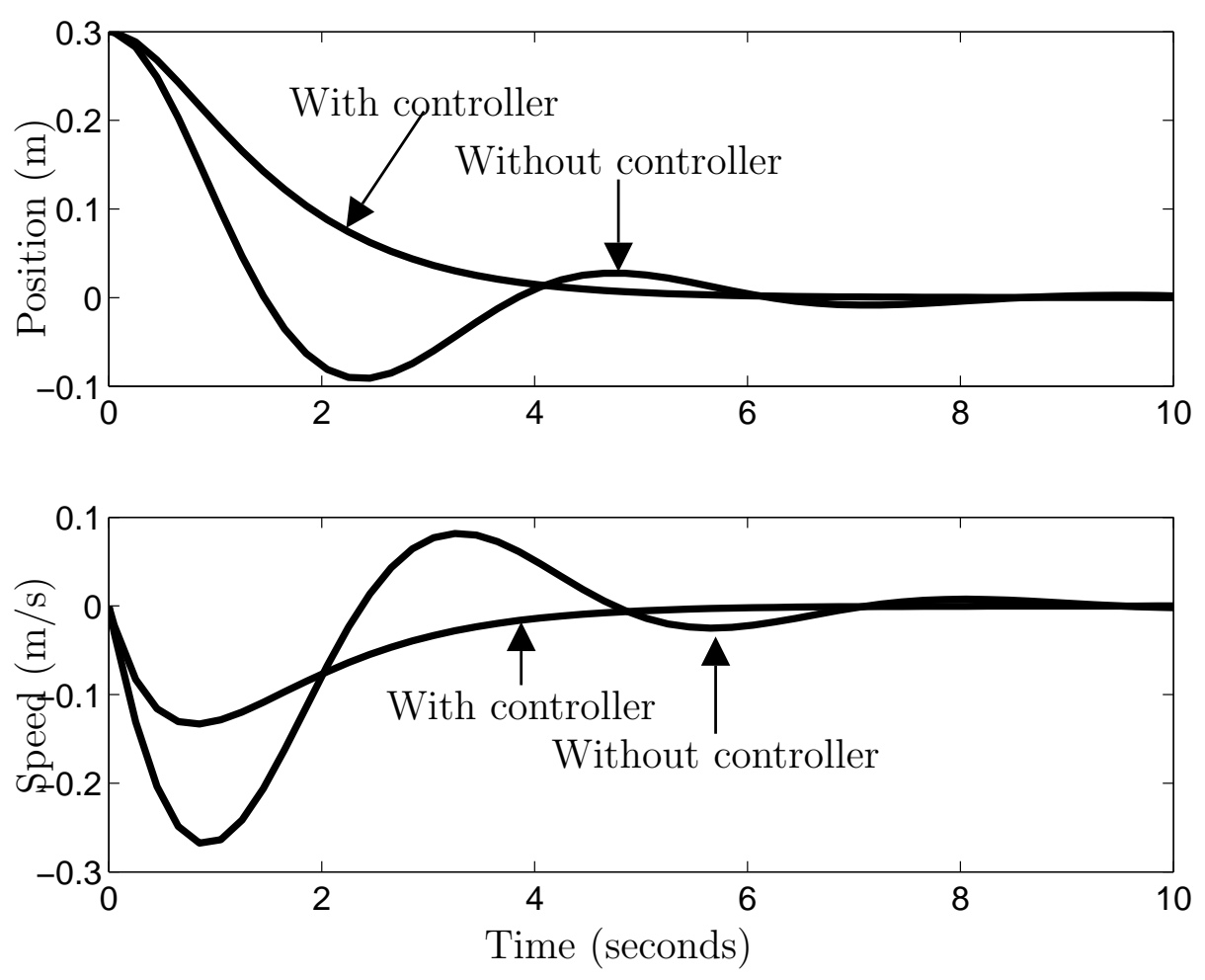

Figure 4.10: Simulation results of the example for a sliding mode controller.

Furthermore, a case in which the system is unstable, i.e. $a_{1}=-2.0, a_{2}=-1.0, b=0.5$ and $c=3.0$ is shown in Figs. 4.12 and 4.13. The system is unstable, so the trajectories of the position and the speed blow out to infinity. The phase plane of these trajectories 
is shown in Fig. 4.13, Fig. 4.12, however, shows that with the sliding mode controller the trajectories converge to the origin.

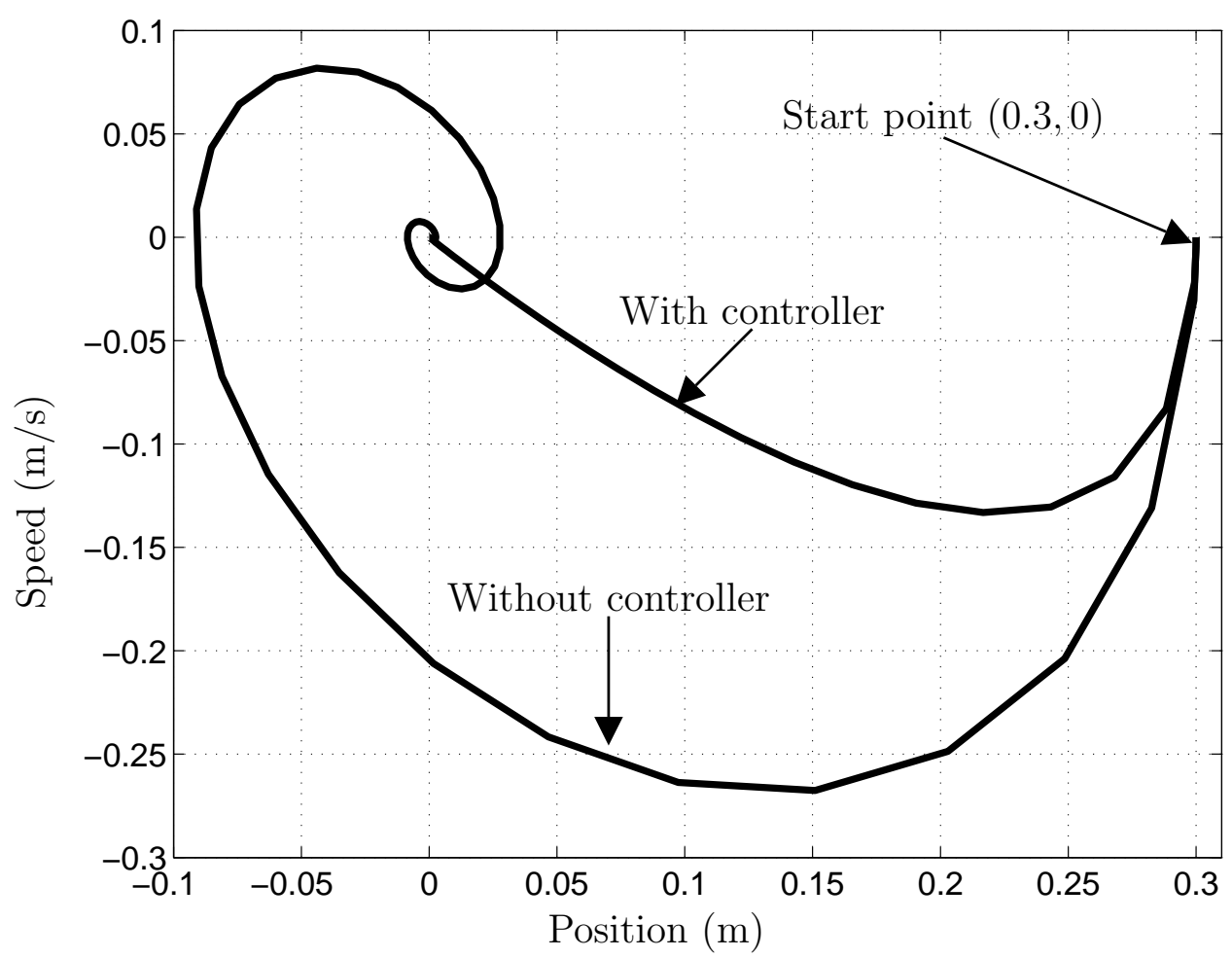

Figure 4.11: Simulation result the phase plane plot for the sliding mode controller example.

\subsection{A Sliding Mode Estimator for the Standpipe}

In this Section, a sliding mode estimator applied to the standpipe of the CFCFB is discussed. The sliding mode estimator is similar to the sliding mode controller in the sense that the controller makes the system drive to certain trajectories, while the estimator makes the system drive to known measured data trajectories. In the standpipe, there are pressure taps to measure pressure drops in the several cells of the standpipe in the CFCFB. The several points are defined as,

$$
\mathrm{z}_{\text {act }}=[0.09,1.0,2.35,2.96,3.47,4.69,6.34,8.2, \text { Top }],
$$


CHAPTER IV. SLIDING MODE ESTIMATION TECHNIQUE
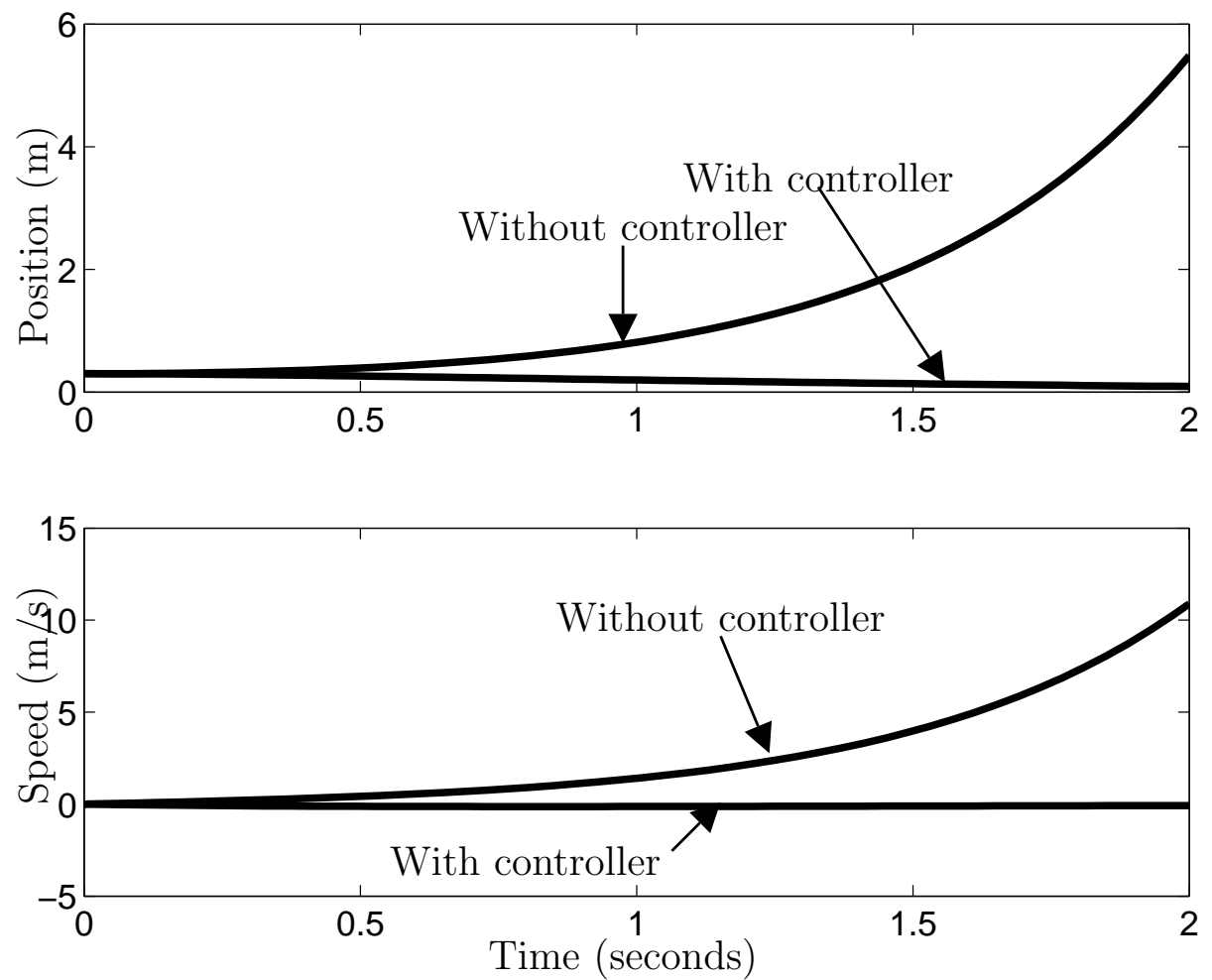

Figure 4.12: Simulation results of a sliding mode control in case of unstable system model.

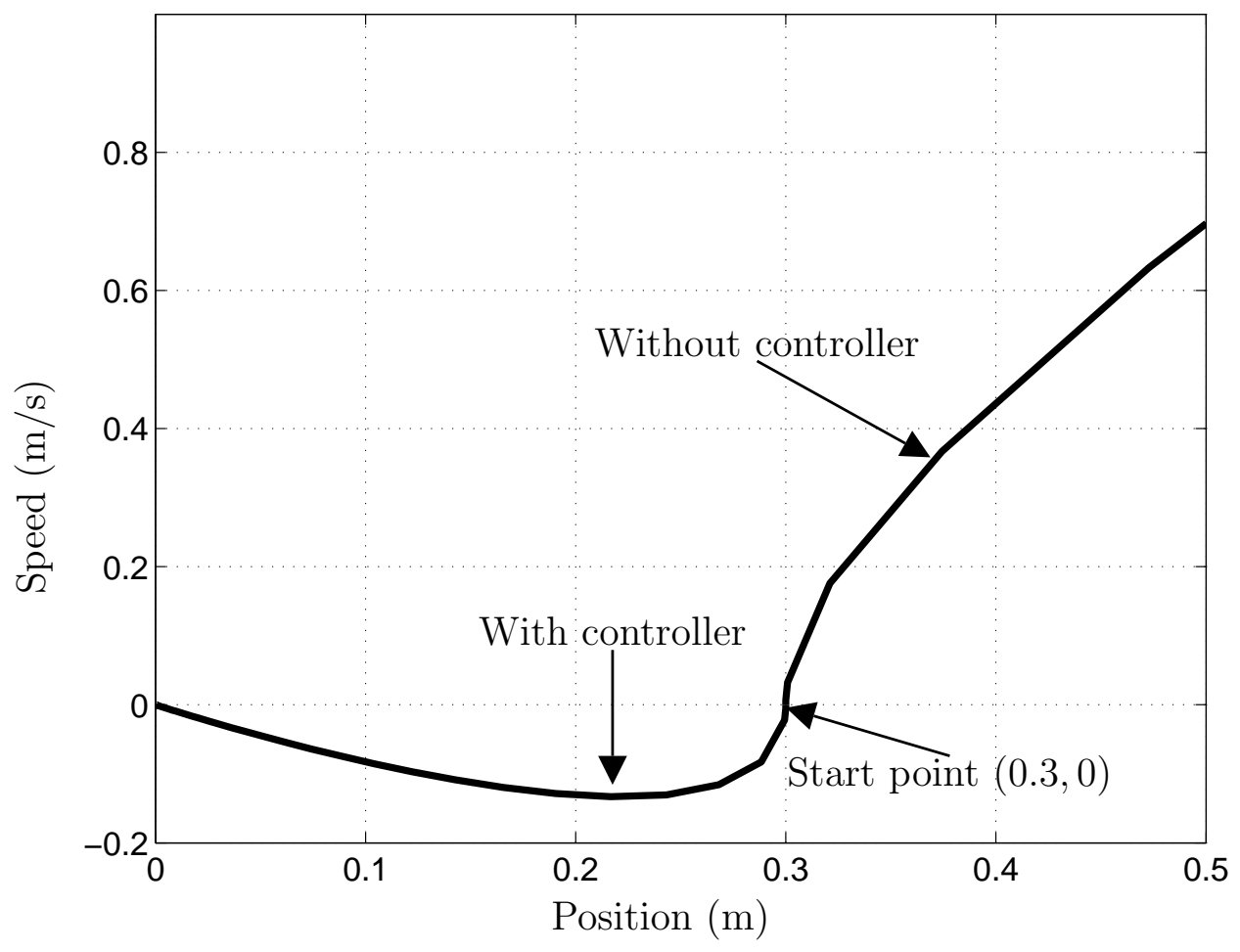

Figure 4.13: Simulation results of the sliding mode with an unstable system model. 
where Top is the highest point of the standpipe, and the units of these points are in meters. The estimated pressure drops are calculated from the first eight points to the top position. Because the discrete cells have certain heights and the height is proportional to the pressure, a proportional pressure is used to calculate the pressure drop profiles in the standpipe. For example, the calculation of the second pressure profile, $\Delta p^{2}$ is shown in Fig. 4.14. The second pressure profile is,

$$
\Delta p^{2}=\Delta \bar{p}_{3}+\Delta p_{t}
$$

where $\Delta \bar{p}_{3}$ is the amount contributed to the second pressure profile in the third discrete cell and $\Delta p_{t}$ is the total pressure sum from the fourth cell to the top cell. If the switching

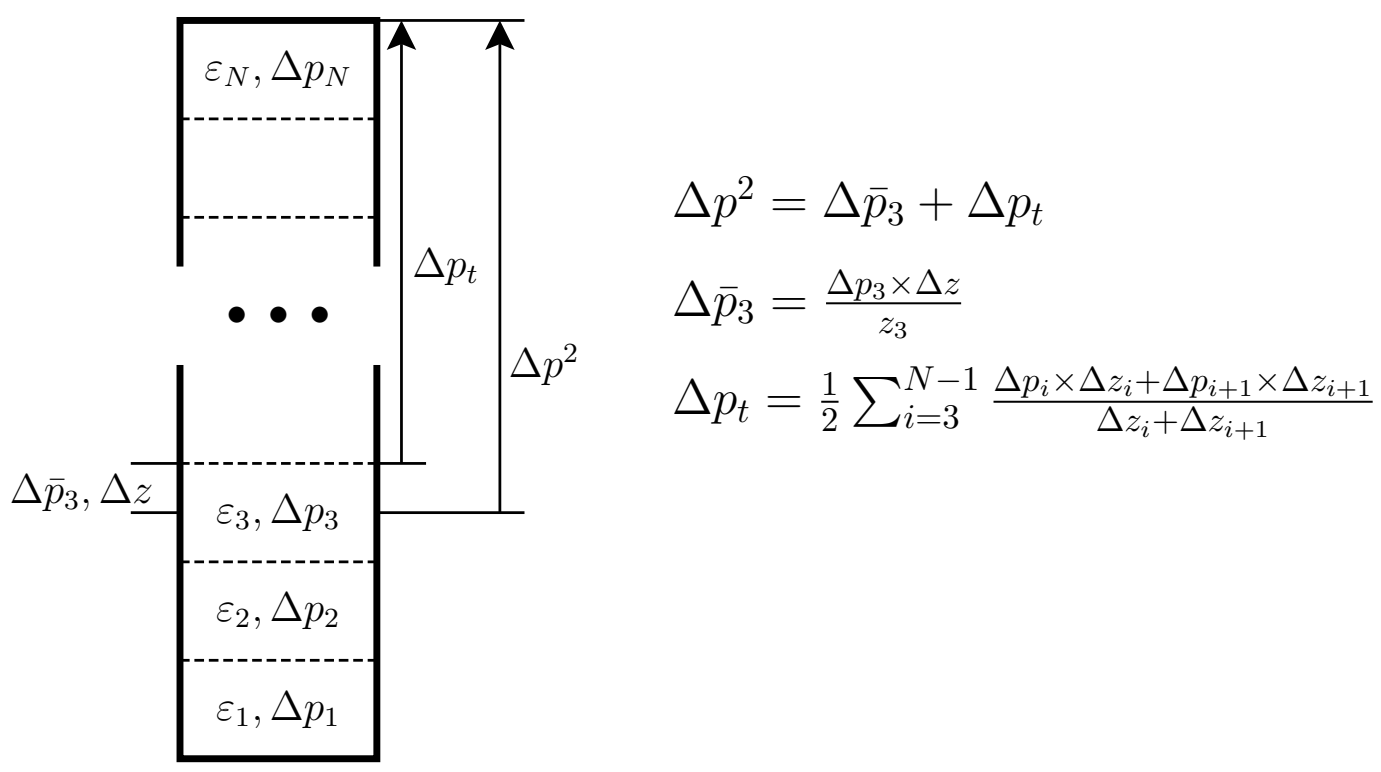

Figure 4.14: Pressure profile calculation for the $\Delta p^{2}$.

surface is defined as,

$$
\Lambda^{j}=K^{j} \operatorname{sgn}\left(p^{j}-\hat{p}^{j}\right), j=1,2, \cdots, N_{p}
$$




\section{CHAPTER IV. SLIDING MODE ESTIMATION TECHNIQUE}

where $K^{j}$ is the gain to be determined, there are $N_{p}$ sliding surfaces to feed back to the state space equation to estimate the states and the pressure outputs. The suggested detail equation of the estimator is given in (4.16).

$$
\hat{\varepsilon}_{k}^{i}=\hat{\varepsilon}_{k-1}^{i}+\Delta t\left(\frac{\hat{j}_{k-1}^{i-}-\hat{j}_{k-1}^{i+}}{\Delta z}+\Lambda^{j}\right) .
$$

The detailed flow chart of the sliding mode estimator algorithm applied to CFCFB is shown in Fig. 4.15. First, the pressure drops need to be measured from the CFCFB facility and

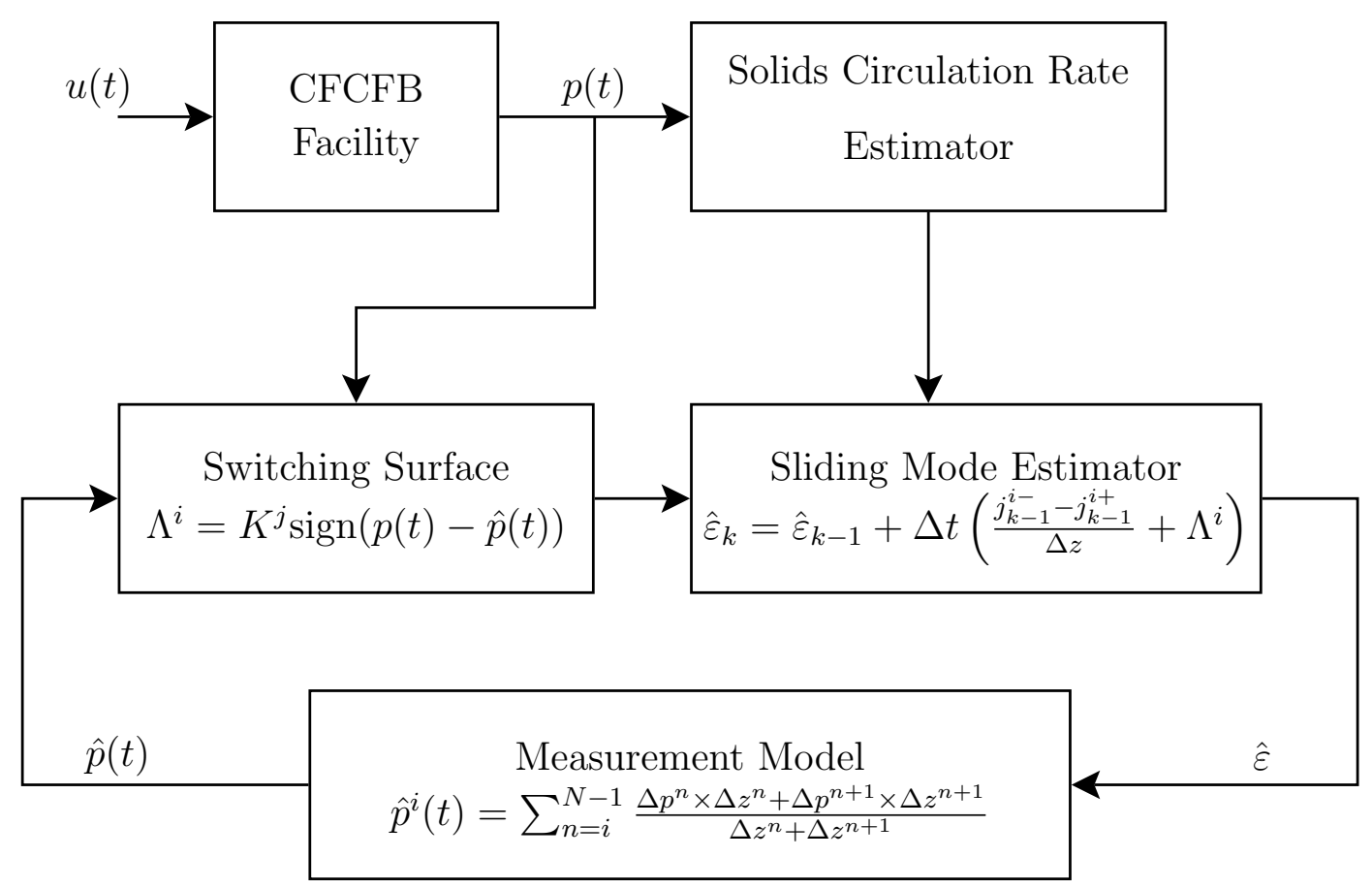

Figure 4.15: Flow chart of the sliding mode estimator.

the switching surface is calculated and the SCR is estimated from the measured pressure drops. The sliding mode estimator then estimates the void fraction using switching surface and estimated SCR. From this void fraction, the estimated pressure drop profiles are found and fed back to the switching surface block. In the switching surface block, the gain is pre-determined using trial and error. 


\subsubsection{Finding the Gain Based on the Lyapunov Stability Criteria}

Let the estimator dynamic equations be approximately,

$$
\frac{\mathrm{d} \varepsilon_{k}^{i}}{\mathrm{~d} t} \simeq \frac{\hat{\varepsilon}_{k}^{i}-\hat{\varepsilon}_{k-1}^{i}}{\Delta t}=\frac{\hat{j}_{k-1}^{i-}-\hat{j}_{k-1}^{i+}}{\Delta z}+\Lambda^{j} \equiv f\left(\hat{\varepsilon}_{k-1}^{i}, t\right)+\Lambda^{j},
$$

the measurement equation is now,

$$
\hat{p}_{k}^{j}=g\left(\hat{\varepsilon}_{k-1}^{i}, t\right) \equiv \sum_{j=i}^{N-1}\left\{\left(z_{j+1}-z_{j}\right) \times\left(C_{1} v_{r}\left(\frac{1-\varepsilon_{k-1}^{j}}{\varepsilon_{k-1}^{j}}\right)^{2}+C_{2}\left(\frac{1-\varepsilon_{k-1}^{j}}{\varepsilon_{k-1}^{j}}\right) v_{r}\left|v_{r}\right|\right)\right\} .
$$

On the other hand, the Lyapunov energy function is defined as,

$$
V(t)=\frac{1}{2} \vec{e}(t)^{T} \vec{e}(t)
$$

where $e^{j}(t)=p^{j}-\hat{p}^{j}$. This energy function is positive, as long as $\vec{e}(t)$ is a real number because it is squared. Furthermore, the derivative of this function should be negative to satisfy the Lyapunov stability condition. The derivative of the energy function is,

$$
\frac{\mathrm{d} V(t)}{\mathrm{d} t}=\vec{e}^{T} \frac{\mathrm{d} \vec{e}}{\mathrm{~d} t}
$$

The derivative of the error function, $\vec{e}(t)$ is found as,

$$
\frac{\mathrm{d} e^{j}}{\mathrm{~d} t}=\frac{\mathrm{d} p^{j}}{\mathrm{~d} t}-\frac{\mathrm{d} \hat{p}^{j}}{\mathrm{~d} t}
$$

Using the chain rule, $\frac{\mathrm{d} x}{\mathrm{~d} y}=\frac{\mathrm{d} x}{\mathrm{~d} t} \frac{\mathrm{d} t}{\mathrm{~d} y}$, the time derivative of pressure can be found as,

$$
\frac{\mathrm{d} \hat{p}^{j}}{\mathrm{~d} t}=\frac{\mathrm{d} \hat{p}^{j}}{\mathrm{~d} \varepsilon^{i}} \frac{\mathrm{d} \varepsilon^{i}}{\mathrm{~d} t}=\frac{\mathrm{d} g}{\mathrm{~d} \varepsilon^{i}} \frac{\mathrm{d} \varepsilon^{i}}{\mathrm{~d} t}
$$

now, the derivative of $\vec{e}$ is

$$
\begin{aligned}
\frac{\mathrm{d} e^{j}}{\mathrm{~d} t} & =\dot{p}^{j}-\frac{\mathrm{d} g}{\mathrm{~d} \varepsilon^{i}} \frac{\mathrm{d} \varepsilon^{i}}{\mathrm{~d} t} \\
& =\dot{p}^{j}-\frac{\mathrm{d} g}{\mathrm{~d} \varepsilon^{i}}\left(f\left(\varepsilon^{i}, t\right)+\Lambda^{j}\right)
\end{aligned}
$$


so, $\dot{V}$ becomes

$$
\begin{aligned}
\dot{V}(t) & =\vec{e}^{T} \dot{\vec{e}} \\
& =\vec{e}^{T} \dot{\vec{p}}-\vec{e}^{T}\left(\frac{\mathrm{d} \vec{g}}{\mathrm{~d} \vec{\varepsilon}} \vec{f}\right)-\vec{e}^{T}\left(\frac{\mathrm{d} \vec{g}}{\mathrm{~d} \vec{\varepsilon}} \mathbf{K} \operatorname{sgn}(\vec{e})\right)<0
\end{aligned}
$$

where the bold face represents the matrix. Therefore, the gain $\mathbf{K}$ should satisfy,

$$
\vec{e}^{T}\left(\frac{\mathrm{d} \vec{g}}{\mathrm{~d} \vec{\varepsilon}}\right) \operatorname{Ksgn}(\vec{e})>\vec{e}^{T} \dot{\vec{p}}-\vec{e}^{T}\left(\frac{\mathrm{d} \vec{g}}{\mathrm{~d} \vec{\varepsilon}}\right) \vec{f} .
$$

The matrix of $\frac{\mathrm{d} \vec{g}}{\mathrm{~d} \vec{\varepsilon}}$ is found as,

$$
\frac{\mathrm{d} \hat{p}^{j}}{\mathrm{~d} \varepsilon^{i}}=\frac{\mathrm{d} g^{j}}{\mathrm{~d} \varepsilon^{i}}
$$

where $j$ runs from 1 to 8 , and $i$ runs from 1 to 25 . This matrix is 8 by 25 in dimension. A detailed expansion is shown in (4.29).

$$
\frac{\mathrm{d} \hat{\vec{p}}}{\mathrm{~d} \vec{\varepsilon}}=\left[\begin{array}{cccccc}
\frac{\mathrm{d} g^{1}}{\mathrm{~d} \varepsilon^{1}} & \frac{\mathrm{d} g^{1}}{\mathrm{~d} \varepsilon^{2}} & \cdots & \frac{\mathrm{d} g^{1}}{\mathrm{~d} \varepsilon^{2}} & \ldots & \frac{\mathrm{d} g^{1}}{\mathrm{~d} \varepsilon^{25}} \\
\frac{\mathrm{d} g^{2}}{\mathrm{~d} \varepsilon^{1}} & \frac{\mathrm{d} g^{2}}{\mathrm{~d} \varepsilon^{2}} & \cdots & \frac{\mathrm{d} g^{2}}{\mathrm{~d} \varepsilon^{2}} & \cdots & \frac{\mathrm{d} g^{2}}{\mathrm{~d} \varepsilon^{25}} \\
\ldots & \ldots & \ldots & \ldots & \ldots & \ldots \\
\frac{\mathrm{d} g^{8}}{\mathrm{~d} \varepsilon^{1}} & \frac{\mathrm{d} g^{8}}{\mathrm{~d} \varepsilon^{2}} & \cdots & \frac{\mathrm{d} g^{8}}{\mathrm{~d} \varepsilon^{2}} & \cdots & \frac{\mathrm{d} g^{8}}{\mathrm{~d} \varepsilon^{25}}
\end{array}\right] .
$$

The gain $\mathbf{K}$ must be determined to satisfy (4.27), but it can not be calculated directly by using the inverse of the matrix. Here, a simple algorithm (i.e. trial and error method) is used to find the gain $\mathbf{K}$ to satisfy (4.27).

\subsection{Simulation Results of the CFCFB}

The simple plot of the standpipe with tags and pressure profiles is depicted in Fig. 4.16. The first state contributes only to the first pressure drop profile; therefore, the correction from the error between measurement and estimated pressure drop profiles should be fed back to the first state. These relationships are represented by numerical values as,

$$
\bar{\varepsilon}=M \times \bar{\Lambda},
$$




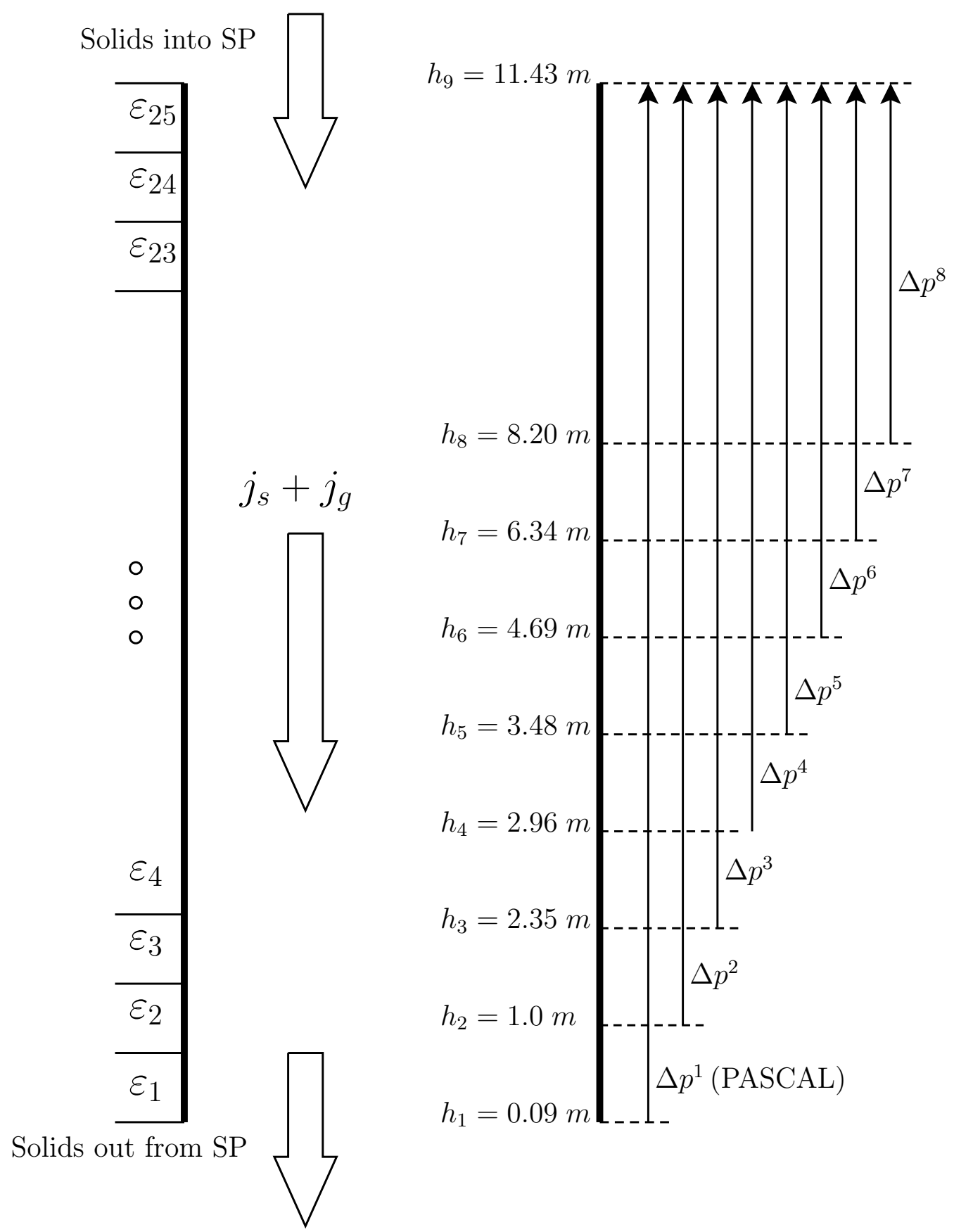

Figure 4.16: Detail representation of the discretized standpipe, the pressure drop profiles, and the solids flow. 


\section{CHAPTER IV. SLIDING MODE ESTIMATION TECHNIQUE}

where $\bar{\varepsilon}$ is the amount contributed to each element of the state vector, $M$ is the matrix that converts the corrections from the sliding mode estimator to the state vector, and $\bar{\Lambda}$ is the error correction from the sliding mode estimator. The estimator dynamic now becomes,

$$
\hat{\varepsilon}_{k}^{i}=\hat{\varepsilon}_{k-1}^{i}+\Delta t\left(\frac{\hat{j}_{k-1}^{i-}-\hat{j}_{k-1}^{i+}}{\Delta z}+\bar{\varepsilon}_{k}^{i}\right) .
$$

In simulation, the standpipe is divided into 25 discrete cells whose outputs are the pressure drop profiles, as shown in Fig. 4.16. The detailed output and input components for the sliding mode estimator are shown in Table 4.1. Two simulations are performed in this

\begin{tabular}{c|c|c|c}
\hline \hline Output tag & Way to obtain & Components & Unit \\
\hline \hline$\Delta p^{1}$ & measured & standpipe 0.09 to top & $\mathrm{N} / \mathrm{m}^{2}$ \\
\hline$\Delta p^{2}$ & measured & standpipe 1.0 to top & $\mathrm{N} / \mathrm{m}^{2}$ \\
\hline$\Delta p^{3}$ & measured & standpipe 2.35 to top & $\mathrm{N} / \mathrm{m}^{2}$ \\
\hline$\Delta p^{4}$ & measured & standpipe 2.96 to top & $\mathrm{N} / \mathrm{m}^{2}$ \\
\hline$\Delta p^{5}$ & measured & standpipe 3.48 to top & $\mathrm{N} / \mathrm{m}^{2}$ \\
\hline$\Delta p^{6}$ & measured & standpipe 4.69 to top & $\mathrm{N} / \mathrm{m}^{2}$ \\
\hline$\Delta p^{7}$ & measured & standpipe 6.34 to top & $\mathrm{N} / \mathrm{m}^{2}$ \\
\hline$\Delta p^{8}$ & measured & standpipe 8.2 to top & $\mathrm{N} / \mathrm{m}^{2}$ \\
\hline$j_{s}$ & estimated & solids circulation rate & $\mathrm{m} / \mathrm{s}$ \\
\hline$j_{g}$ & measured & move air & $\mathrm{m} / \mathrm{s}$ \\
\hline$h$ & measured & bed-height & $\mathrm{m}$ \\
\hline \hline
\end{tabular}

Table 4.1: Output and input tags and their components of the sliding mode estimator.

research. The first case is shown in Fig. 4.17 and Fig. 4.18. Fig. 4.17-a shows the instantaneous void fraction versus the standpipe height while Fig. 4.17-b depicts the instantaneous measured and estimated pressure profiles versus the standpipe height. The simulation results running to 300 seconds for measured and estimated bed-heights versus time are shown in Fig. 4.18. The solid lines with asterisks represent the simulation results from the sliding mode estimator while the solid lines with circles show the measured values. The other case is shown in Fig. 4.19 and Fig. 4.20. Fig. 4.19-a illustrates the instantaneous void fractions versus the standpipe height while the result of the measured and estimated pressure pro- 
files versus standpipe height is shown in Fig. 4.19-b. Fig. 4.20 shows bed-height versus time when the simulation continues to 300 seconds. Since the measurements are taken in 15 second intervals, the estimated bed-heights are also calculated every 15 seconds. In the beginning of the bed-height estimation, there are some overshoots because the estimator takes time to converge. It is worth mentioning that the sliding gain needs to be determined for each simulation cases to have better simulation results.

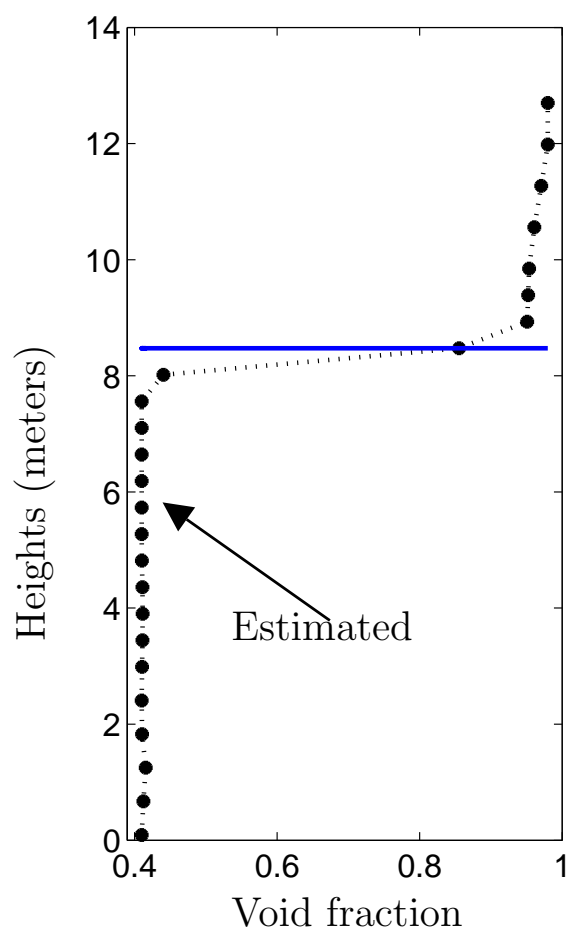

(a) Void fraction vs. height

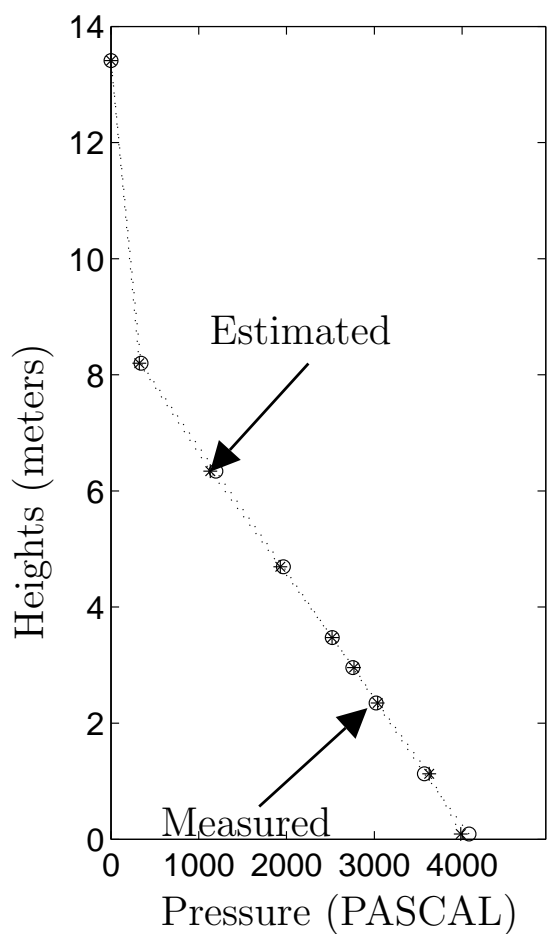

(b) Pressure profile vs. height

Figure 4.17: Instant void fraction and pressure vs. height of the standpipe.

\subsection{Summary}

A sliding mode estimator applied to the standpipe of the CFCFB was introduced and discussed in this Chapter. The sliding mode estimator needs the dynamic model discussed in Chapter II, as well as the solids circulation rate measurable from the spiral instrument 


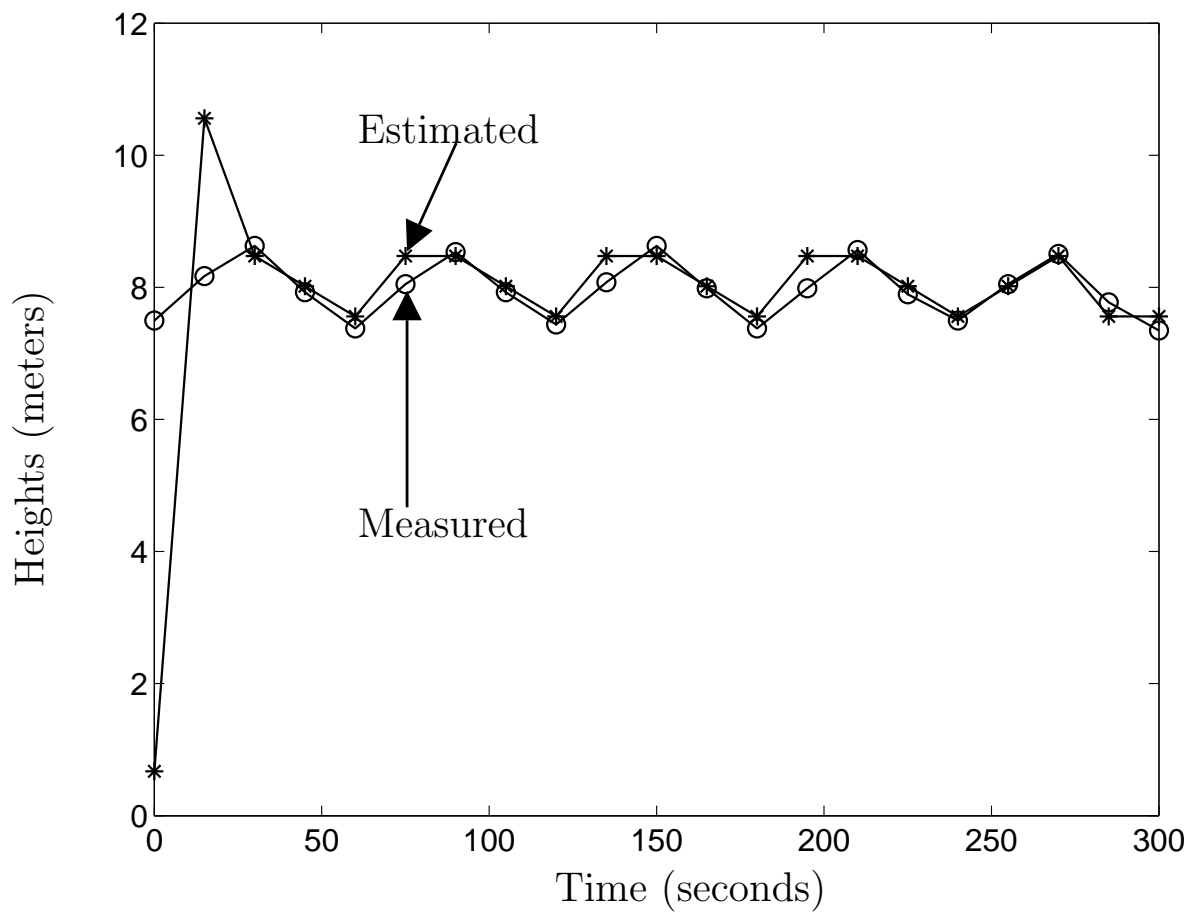

Figure 4.18: Simulation results of a sliding mode estimator applied to the CFCFB.

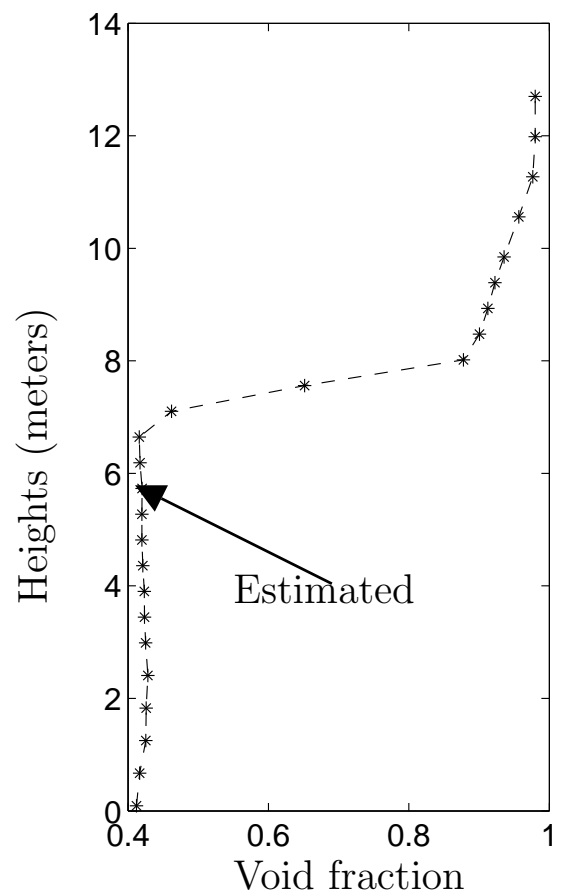

(a) Void fraction vs. height

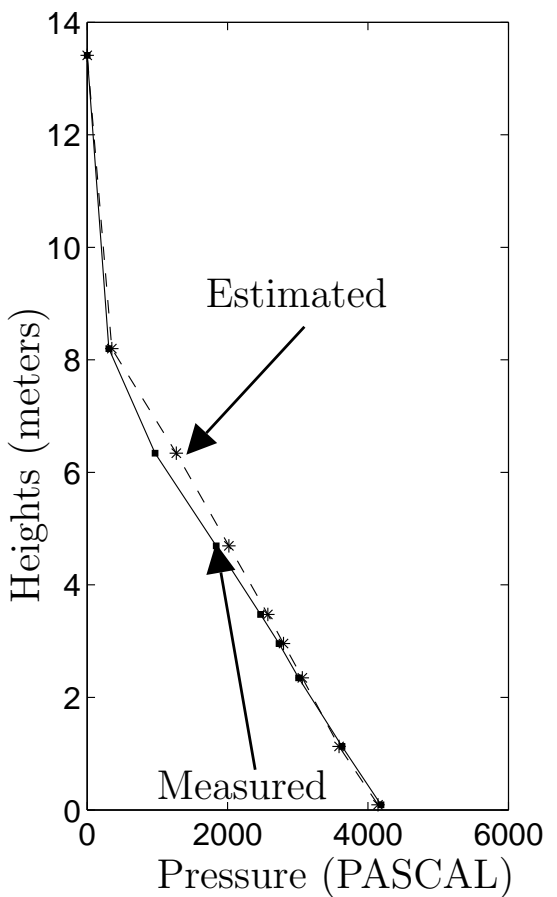

(b) Pressure profile vs. height

Figure 4.19: Instant void fraction and pressure vs. the height of the standpipe. 


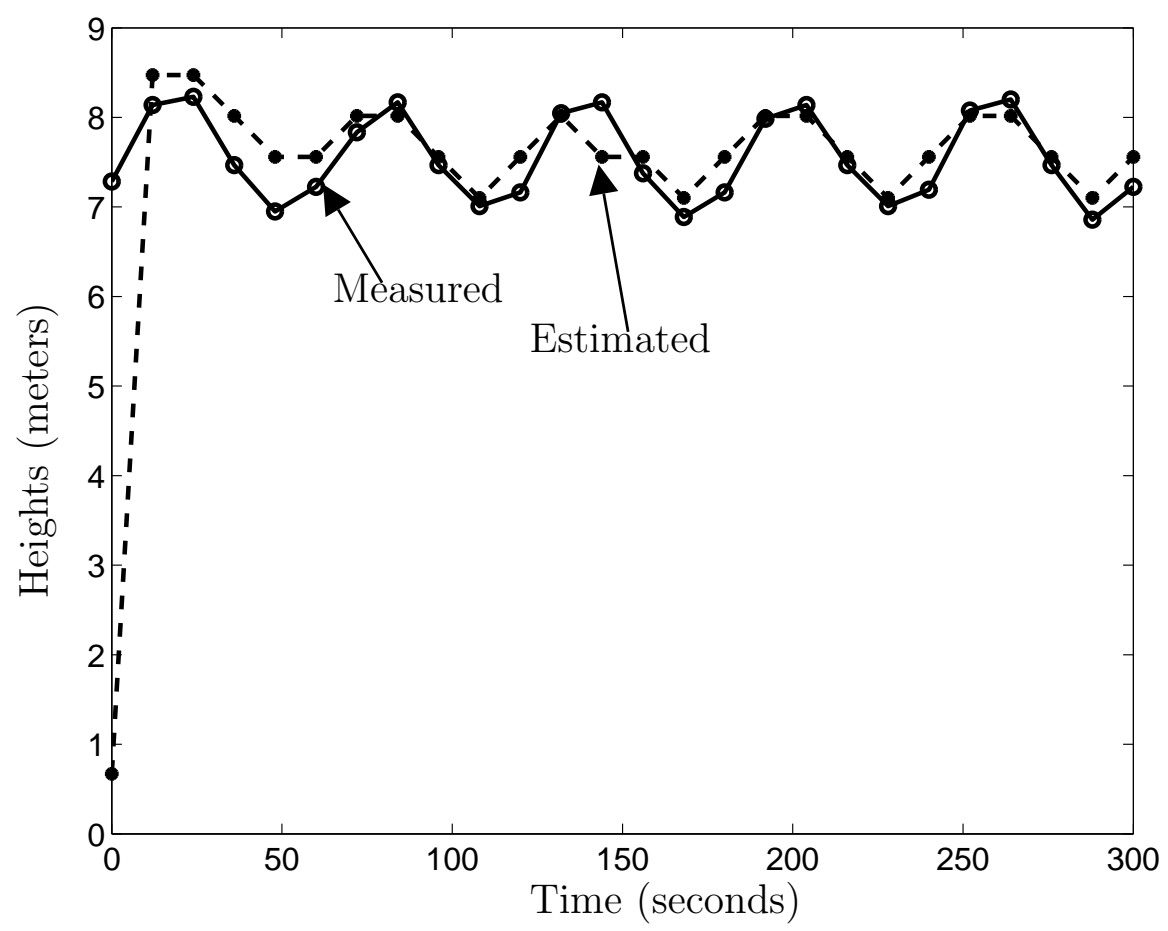

Figure 4.20: Simulation results of a sliding mode estimator applied to the CFCFB.

in the standpipe. To explain the sliding mode estimator, the sliding mode controller was first introduced and explained. According to the basic idea of the sliding mode controller, as well as that of the estimator, both the saddle and center points are used to explain the sliding mode controller. One example of the sliding mode controller design procedure was explained and discussed. In the $\mathrm{CFCFB}$ application, the sliding mode estimator was applied in order to estimate the void fractions, and hence the bed-height. The results show this method can estimate the state and bed-height correctly. 


\section{Chapter V}

\section{Design Linear Controllers for both the SCR and the Bed-height}

\subsection{Introduction}

Previously in Chapter III, the solids circulation rate and the bed-height were successfully estimated and calculated with the experimentally collected data sets. In this Chapter, linear control theory will be applied in order to control these quantities since there are linear relationships between the move air (control variable) and the SCR and/or bed-height. A mathematical model of the entire CFCFB system has not yet been developed completely, but the neural network system was used in an attempt to model the entire CFCFB, Koduru [10]. In this research, the neural network with a back-propagation algorithm is used to model the entire CFCFB system, using the pressure drops as input and output, together with the move air and the aeration flow in the riser as an input. Simulations are run for step changes for both the SCR and the bed-height. 


\subsection{Control Variable and Outputs}

To illustrate the relationships between the control variable (move air) and outputs (SCR and bed-height), control variable versus output plots are shown in Figs. 5.1 to Fig. 5.4. The first two figures show the relationship between move air and SCR in such a way that these two variables are in linear arrangement, such that, as the move air increases the

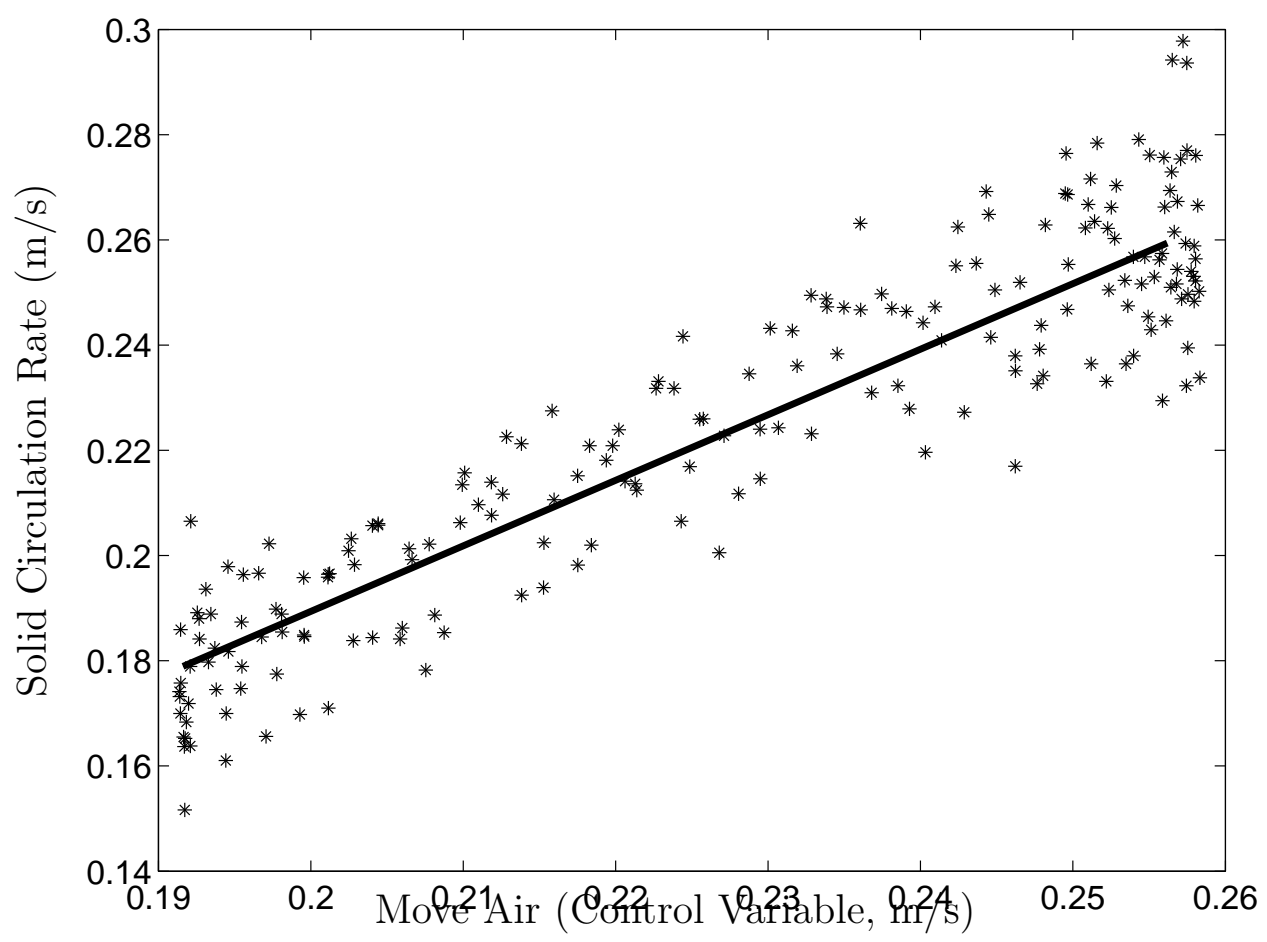

Figure 5.1: Plot representing the linear relationship between move air and the SCR.

SCR increases. Some noise components are included. The same phenomenon happens in Figs 5.3 and 5.4 for move air and bed-height. In this case, as the move air increases the bed-height decreases. For both the SCR and bed-height, the relationships could be represented by linear equations as,

$$
\begin{array}{ll}
\mathrm{SCR} & =\gamma_{1} \times \text { move air }+\gamma_{2}+\text { noise components, } \\
\text { bed-height } & =\gamma_{3} \times \text { move air }+\gamma_{4}+\text { noise components, }
\end{array}
$$

where $\gamma_{1}, \gamma_{2}, \gamma_{2}$, and $\gamma_{4}$ are arbitrary constant. 


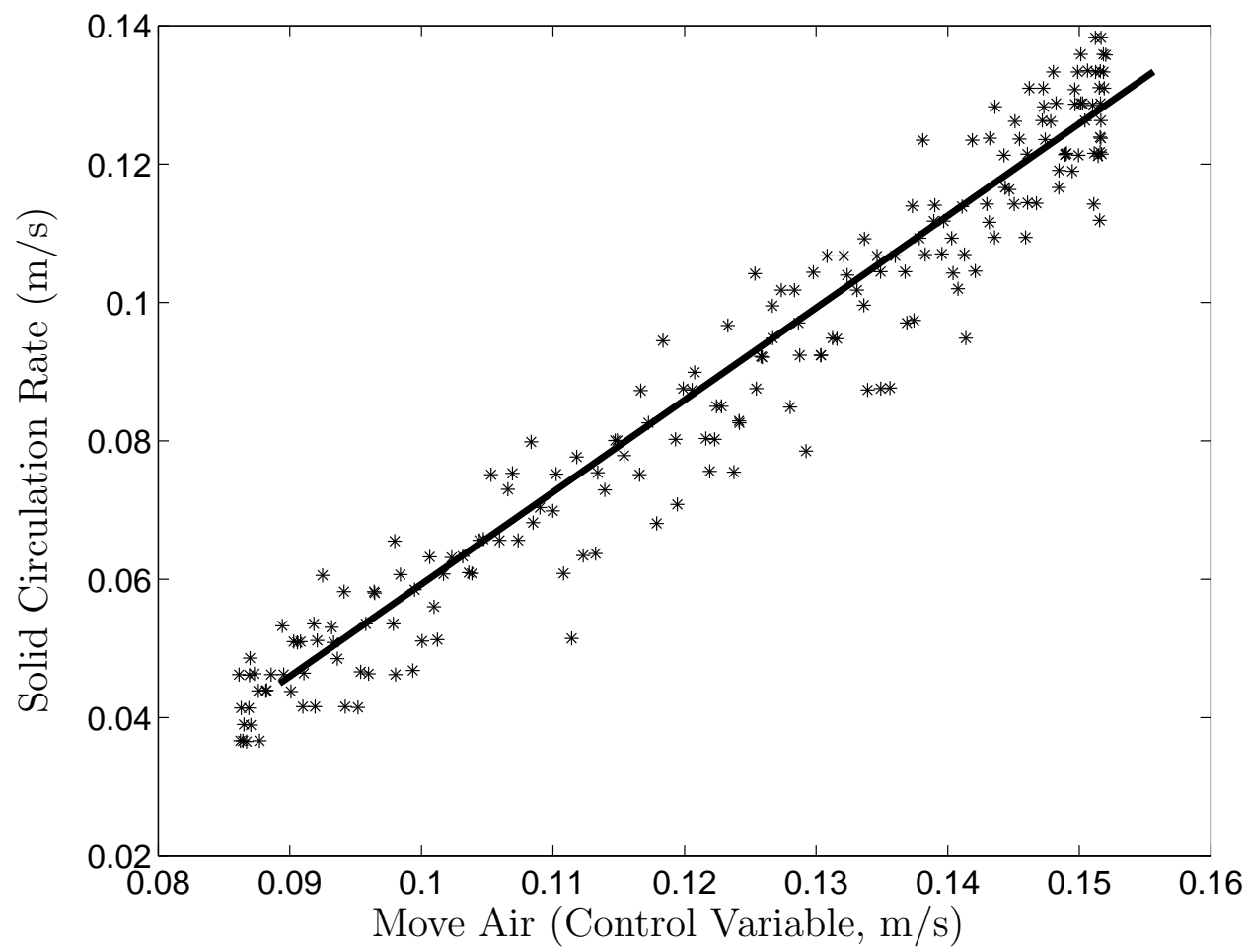

Figure 5.2: Plot representing the linear relationship between move air and SCR.

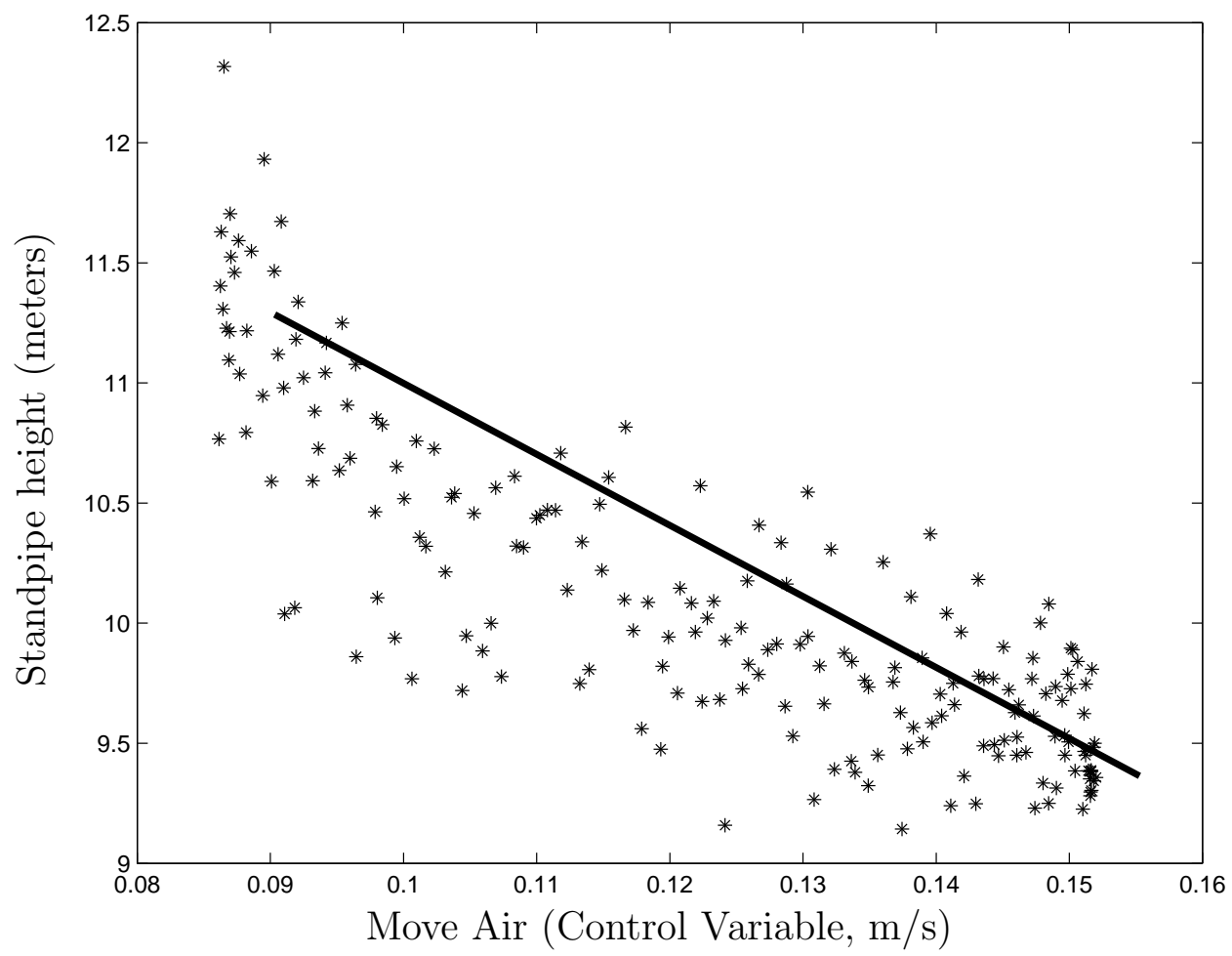

Figure 5.3: Plot representing the linear relationship between move air and bed height. 


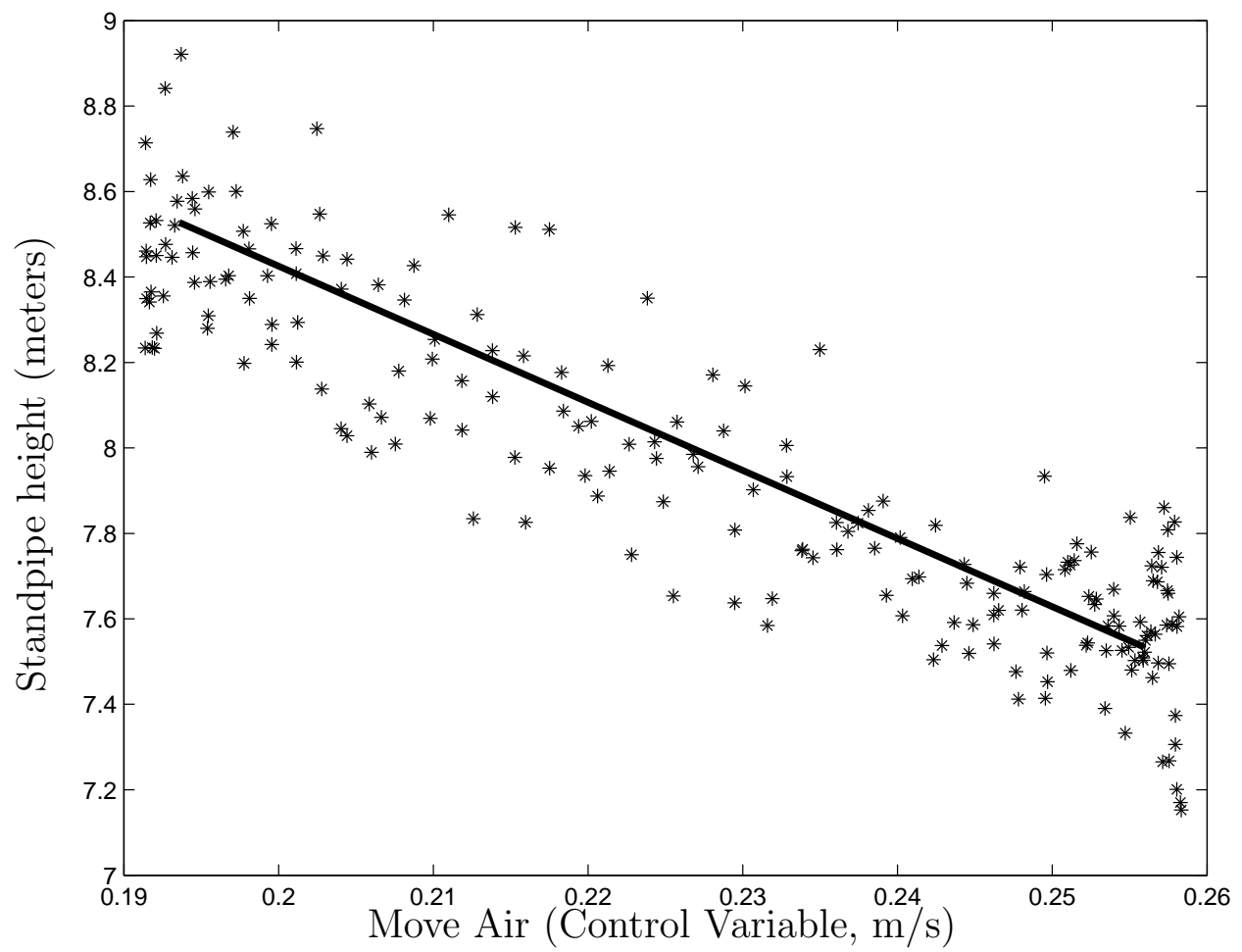

Figure 5.4: Plot representing the linear relationship between move air and bed height.

\subsection{Neural Network Methods}

In this research, the neural network with a back-propagation algorithm is applied in order to build the system model for linear control and simulation purposes. The neural network has been used for system modeling with non-linear systems, Pantino and Liu [41], Chen and Xi [42], Hutchins [43], Antsaklis [44], Owens [45], Fernandez et al. [46]. These papers used a neural network with a back-propagation algorithm combined with other advanced methods to model and control nonlinear systems. In these studies, the back-propagation algorithm was applied in order to train the neurons that use the tansig functions for the hidden layers and purelin functions for the output layers. 


\subsubsection{Neural Network Application to the CFCFB}

To train the neural network, the architecture of the neural network needs to be determined. To obtain the best performance from several trials, one hidden layer and 15 input neurons, 10 hidden neurons, and 10 output neurons are selected. The LevenbergMarquardt back-propagation algorithm is used for building the system model, Hagan and Menhaj [47], Hagan et al. [63]. The Levenberg-Marquardt method is a second-order training speed without computing the Hessian matrix (second derivative). The Hessian matrix is approximated as,

$$
\mathbf{H}_{n}=\mathbf{J}_{n}^{T} \mathbf{J}_{n},
$$

where $\mathbf{H}_{n}$ is the Hessian matrix containing the second order and $\mathbf{J}_{n}$ is the Jacobian matrix containing the first order derivative of the network errors with respect to the weights and biases. The gradient is computed as,

$$
\mathbf{g}_{n}=\mathbf{J}_{n}^{T} \mathbf{e}
$$

The Levenberg-Marquardt algorithm uses as an approximated Hessian matrix to update the states, as in Hagan et al. [63], Demuth [64],

$$
x_{n}(k+1)=x_{n}(k)-\left[\mathbf{J}_{n}^{T} \mathbf{J}_{n}+\mu_{n} \mathbf{I}_{n}\right]^{-1} \mathbf{J}_{n}^{T} \mathbf{e} .
$$

where $x_{n}(k)$ is the neuron state function and $\mu_{n}$ is the constant number. The detailed inputs and targets definitions are shown in Table 5.1. To speed up the training time, the inputs and outputs are normalized by the following equations,

$$
\begin{aligned}
u(k) & =\frac{u(k)-\bar{u}(k)}{\max (u(k))-\min (u(k))}, \\
T(k) & =\frac{T(k)-\bar{T}(k)}{\max (T(k))-\min (T(k))},
\end{aligned}
$$

where $\bar{u}(k)$ is the middle point of the maxima and minima of $u(k)$, and $\bar{T}(k)$ is the middle point of the maxima and minima of $T(k)$. These steps are adapted because the transfer 


\begin{tabular}{l|c||l|c}
\hline \hline Input & Component at $t-1$ & Target & Component at $t$ \\
\hline$u_{1}(k)$ & riser total pressure drop & $T_{1}(k)$ & riser total pressure drop \\
\hline$u_{2}(k)$ & standpipe $0.3 \mathrm{~m}$ top & $T_{2}(k)$ & standpipe $0.3 \mathrm{~m}$ to top \\
\hline$u_{3}(k)$ & standpipe $3.7 \mathrm{~m}$ to top & $T_{3}(k)$ & standpipe $3.7 \mathrm{~m}$ to top \\
\hline$u_{4}(k)$ & standpipe $7.7 \mathrm{~m}$ to top & $T_{4}(k)$ & standpipe $7.7 \mathrm{~m}$ to top \\
\hline$u_{5}(k)$ & standpipe $9.7 \mathrm{~m}$ to top & $T_{5}(k)$ & standpipe $9.7 \mathrm{~m}$ to top \\
\hline$u_{6}(k)$ & standpipe $11.4 \mathrm{~m}$ to top & $T_{6}(k)$ & standpipe $11.4 \mathrm{~m}$ to top \\
\hline$u_{7}(k)$ & standpipe $15.4 \mathrm{~m}$ to top & $T_{7}(k)$ & standpipe $15.4 \mathrm{~m}$ to top \\
\hline$u_{8}(k)$ & standpipe $20.67 \mathrm{~m}$ to top & $T_{8}(k)$ & standpipe $20.67 \mathrm{~m}$ to top \\
\hline$u_{9}(k)$ & standpipe $26.9 \mathrm{~m}$ to top & $T_{9}(k)$ & standpipe $26.9 \mathrm{~m}$ to top \\
\hline$u_{10}(k)$ & move air at $t$ & $T_{10}(k)$ & solids circulation rate \\
\hline$u_{11}(k)$ & riser aeration at $t$ & & \\
\hline$u_{12}(k)$ & solids circulation rate at $t$ & & \\
\hline \hline
\end{tabular}

Table 5.1: Definitions for neural network training with inputs and outputs.

function, tansig for the back-propagation is,

$$
\operatorname{tansig}(k)=\frac{2}{1+e^{-2 \times u(k)}}-1
$$

where $\operatorname{tansig}(k)$ is the transfer function and $u(k)$ is the input of a neuron. The output layer, however, uses a different function, a pure linear function to make the training procedure faster, thus,

$$
\operatorname{purelin}(k)=u(k)
$$

where $u(k)$ is the input of a neuron. The detailed training schematic diagram is shown in Fig. 5.5. In the output layer, the neurons are updated by errors calculated from the target and synthesized outputs. These steps go back to the hidden layer, and the same update procedure runs to the neurons. The updating procedure is done at the input layer. In the next step, the synthesized outputs are calculated and back updating procedures are performed. These procedures continue until the targeted errors reach the acceptable value that user determines. 


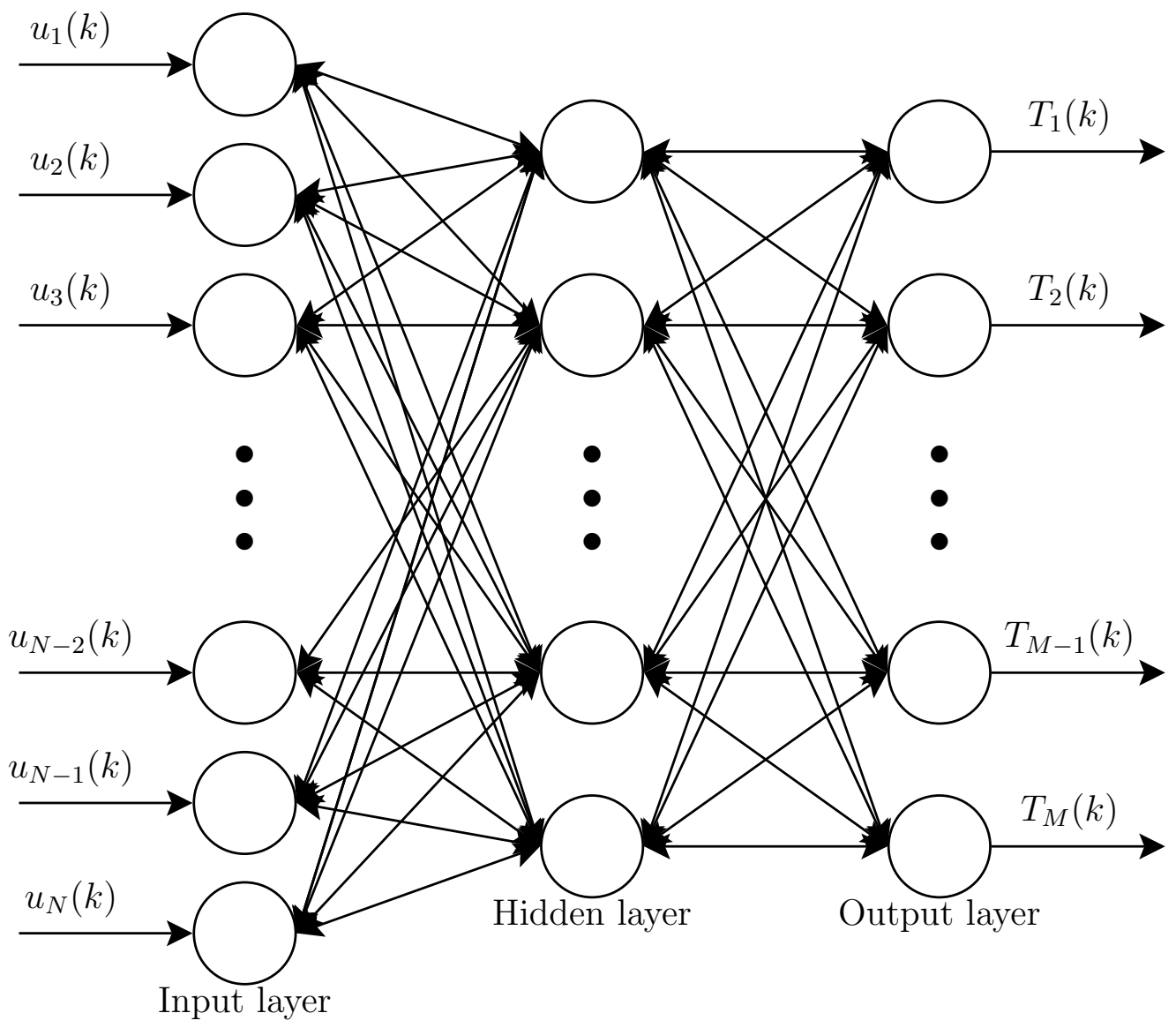

Figure 5.5: The detailed schematic diagram for the training procedure of the back propagation algorithm

\subsubsection{Results of Neural Network Application}

The simulation results from applying the neural network to the CFCFB are shown in Figs. 5.6 and 5.7. Both plots show the measured and synthesized solids circulation rates. The solid lines represent the SCR from the spiral measurement while dash line is the synthesized SCR from the neural network system. Both synthesized SCRs are close to the measured values, and demonstrate the effectiveness of neural network system model. 


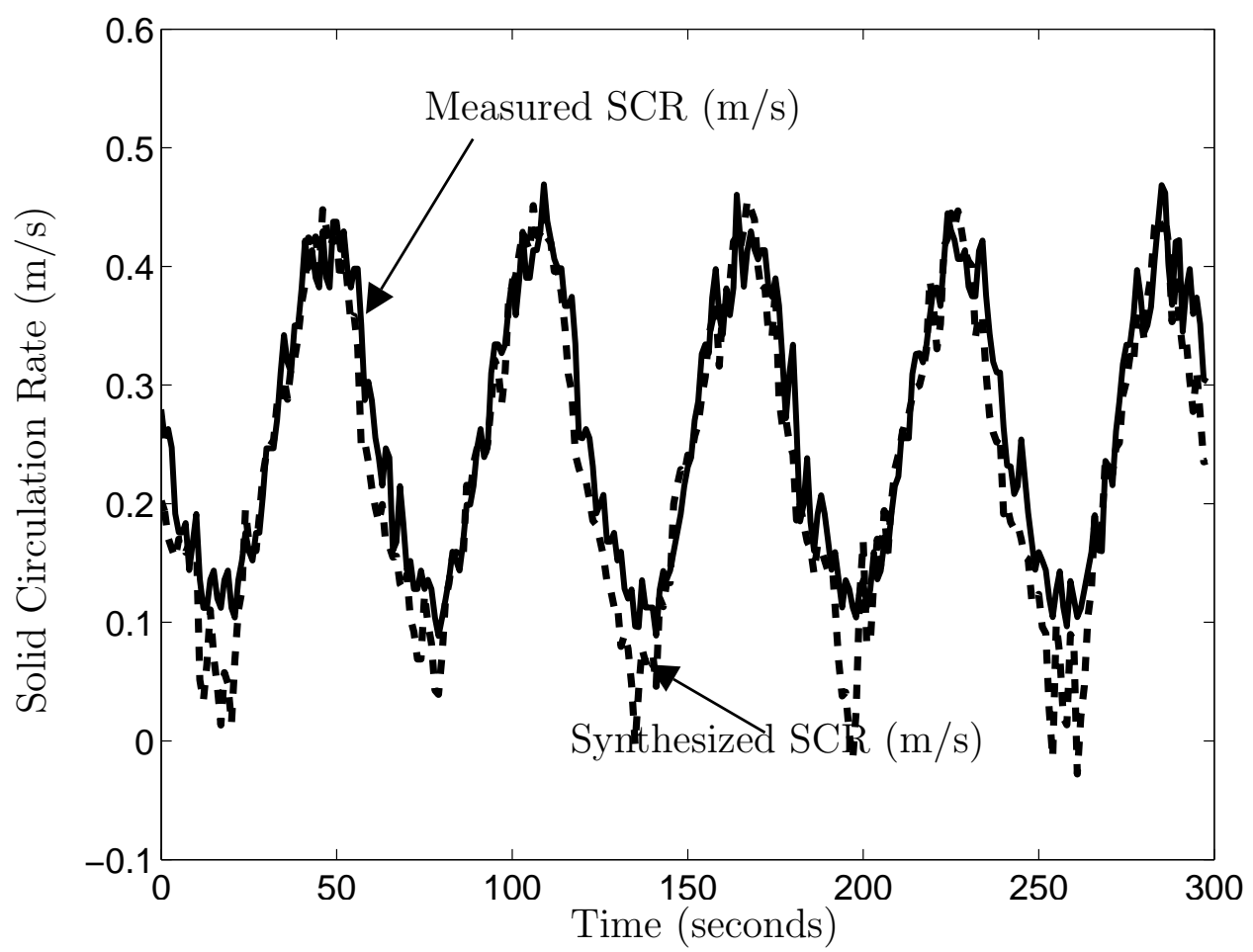

Figure 5.6: The SCR measured and synthesized from neural network.

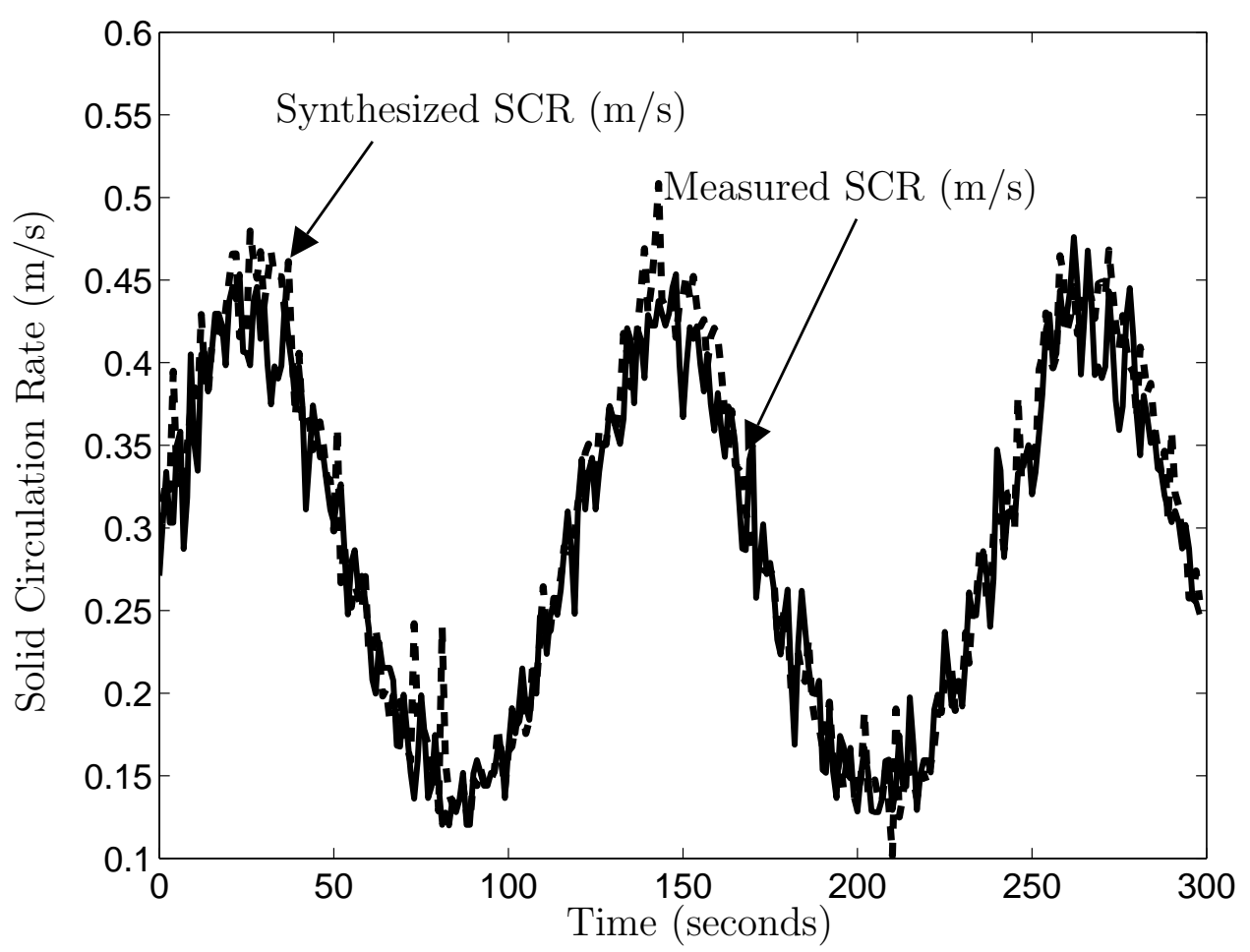

Figure 5.7: The SCR measured and synthesized from neural network. 


\subsection{Linear Controller Design}

Fig. 5.8 illustrates a basic simulation diagram of the linear controller of the solids circulation rate and the bed-height with a neural network system model. It consists of a neural network system, an estimator for the SCR or calculator of the bed-height from the pressure drops, and the linear controller. Once the pressure data is available from the

Estimated SCR or calculated bed height

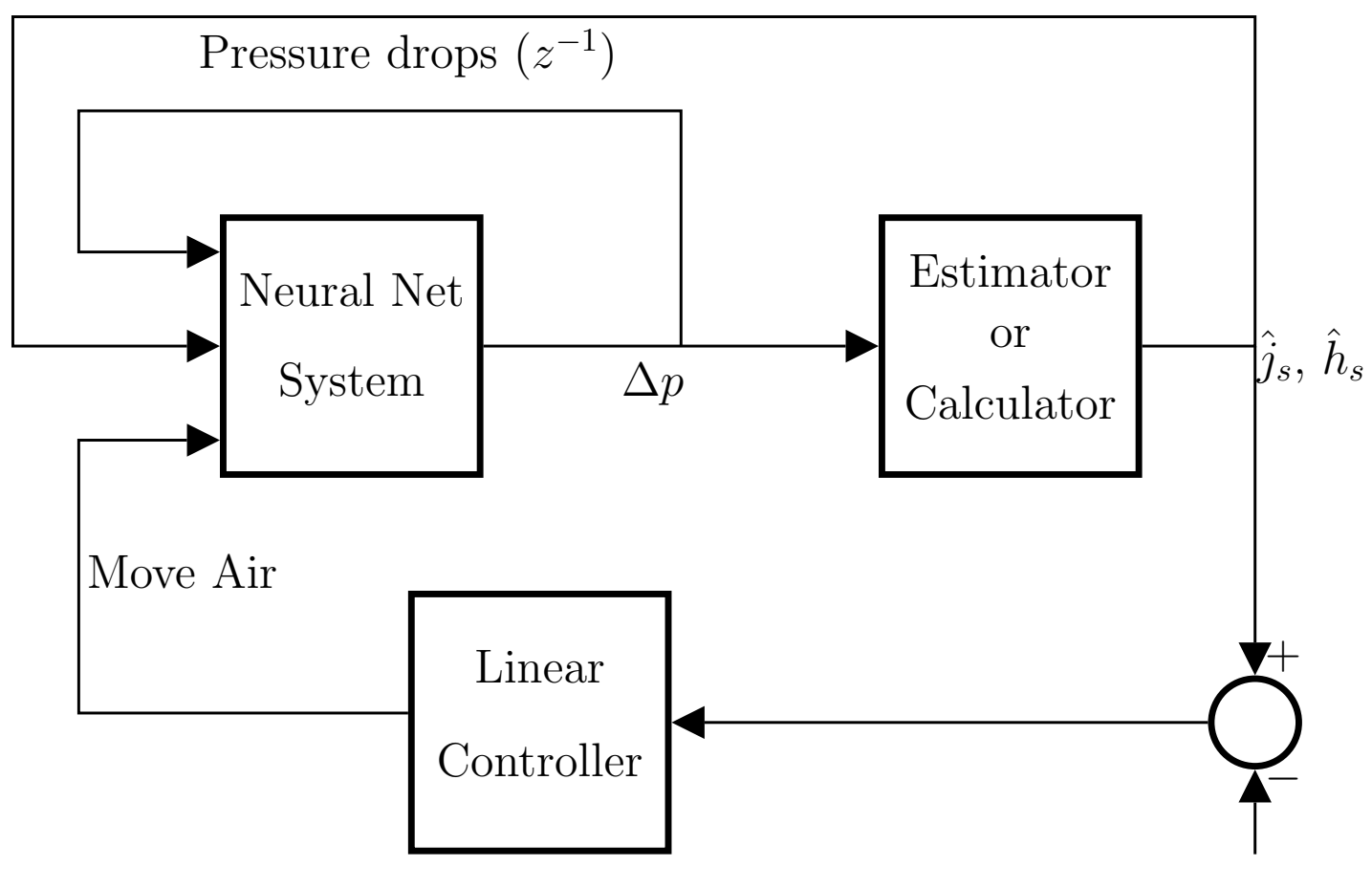

Set Point

Figure 5.8: Block diagrams for the linear and system models with a neural network.

neural network, the Kalman estimator can estimate the solids circulation rate at time, $t$. This rate is compared with the set point, while the linear controller generates the command signal to adjust the control variable (move air). In a linear controller block, the control signal is generated from the error, then this signal adds up with previous time step move 
air to adjust the solid circulation rate. The move air is now fed back to the neural network system with time lagged pressure data produced by the neural network system at time $t$. In the simulation, initial conditions need to be determined to simulate further cases. Once the initial conditions have been determined, step changes for a desired set point of the solids circulation rate are made. The bed-height simulation follows the same procedure as that of the SCR control. In this case, the estimator is merely replaced with the bed-height calculator discussed in Section, 3.6 of Chapter III.

\subsection{Simulation Results of Controllers}

The simulation results with the neural network system model estimating the solids circulation rate using a Kalman filter with a linear controller are shown in Fig. 5.9 for the desired and estimated trajectories as well as for the control input. Initially, the system controller runs and finds the proper initial condition for the Kalman filter and the state for the estimation system. The controller was applied for about 10 seconds to decrease the SCR set point by about $0.03 \mathrm{~m} / \mathrm{s}$, and about 50 seconds after the set point of the solids circulation rate increases by $0.05 \mathrm{~m} / \mathrm{s}$. The estimated solids circulation rate follows well with the set point as it changes. The control input (move air) also has the same shape as the set point does. Since the neural network system cannot be used outside of the range from 0.2 to $0.4 \mathrm{~m} / \mathrm{s}$ for move air, the simulation can only be conducted in this range.

For the bed-height control simulation, Fig. 5.10 shows the calculated and the desired bed-heights versus time, as well as the control input versus time. Like the SCR estimation, the bed-height step changes occur at about 5 seconds decreasing by 0.2 meters and at about 50 seconds increasing by 1 meter. The calculated bed-height follows well with the desired set values. In this case, the control input has the opposite shape, as seen in Fig. 5.10, since the relationship between bed-height and move air is negative, i.e. as move air increases the 

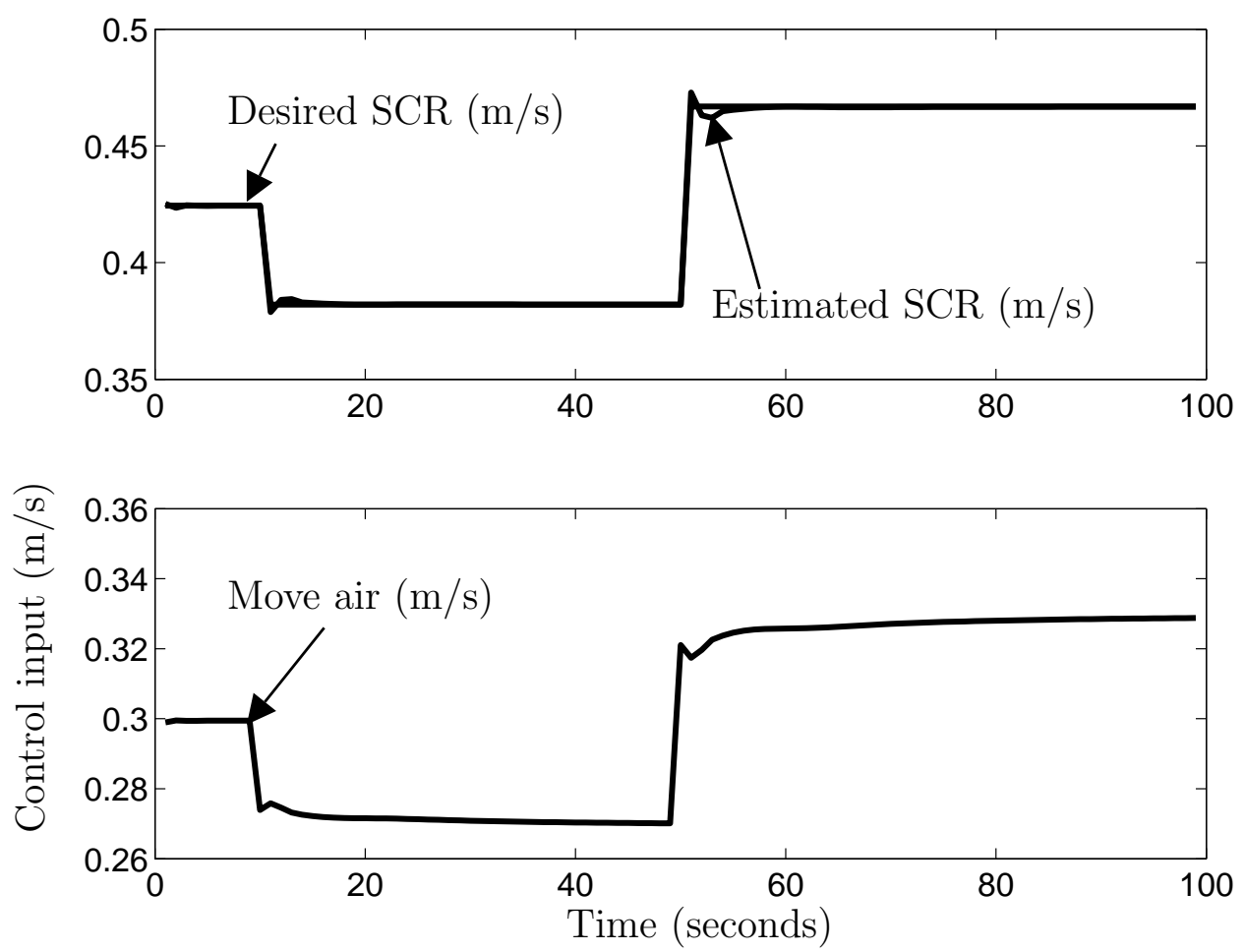

Figure 5.9: Simulation result of the SCR with the linear controller.

bed-height decreases.

\subsection{Summary}

Control of the SCR and the bed-height, as well as building the entire CFCFB system model using neural network, were discussed in this Chapter. The relationships between control input and outputs were investigated and determined to be linear. Since there are linear relationships between the move air and the SCR and/or the bed-height, a linear controller was applied in order to regulate these two quantities. There are no complete mathematical or dynamic models available for the entire CFCFB system so a neural network was applied to model the entire system. The simulation results for the neural network system model are shown and discussed. The tests for both control simulations were performed for the step changes; results show that the regulations of the SCR and bed-height 
CHAPTER V. DESIGN LINEAR CONTROLLER FOR THE SCR AND BED-HEIGHT
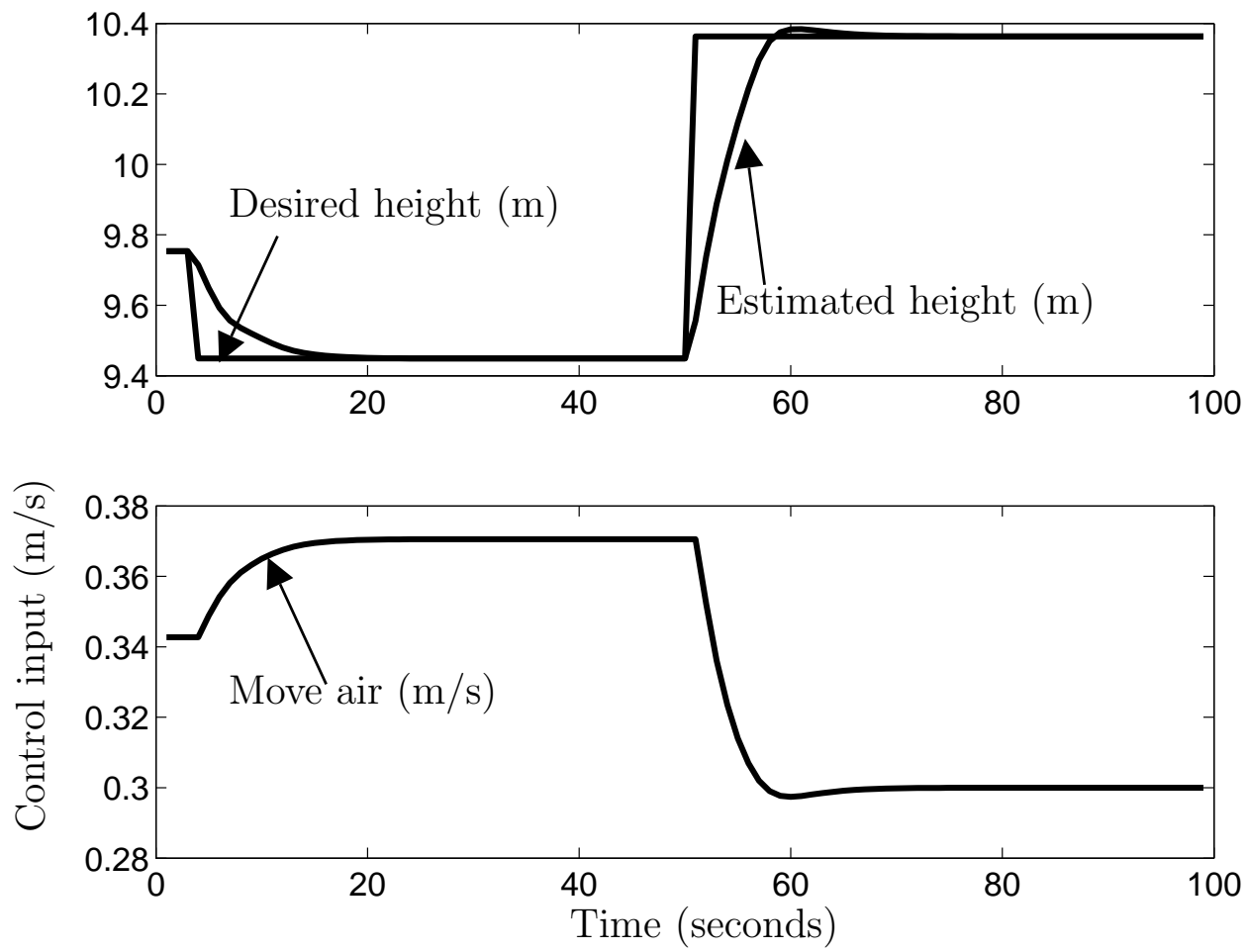

Figure 5.10: Simulation result of the bed-height control with the linear controller.

using a linear controller were acceptable and satisfactory. 


\section{Chapter VI}

\section{Conclusions and Future Work}

\subsection{Conclusions}

The detailed design and operation of a cold flow circulating fluidized bed (CFCFB) installed at National Energy Technology Laboratory in Morgantown, WV has been studied and modeled. The standpipe dynamic model based on the continuity equation using void fraction as a state variable has been presented and discussed. This dynamic model discretizes the standpipe into several small cells, and each void fraction is isolated. The model uses the Richardson and Zaki correlation to relate the void fraction to the relative gas velocity in the several zones. Depending on the wave speed, the gas flux can be either up or down. The measurement model of the standpipe is the integrated version of the Ergun equation using the trapezoidal rule.

The linear discrete system model used for estimating the solids circulation rate using the Kalman filter was developed in this dissertation. The least squares estimation and an extended observability matrix were applied in order to find the matrices $A, B$, and $C$ in the state space model. After finding these matrices, the system was transformed to another form that did not include fictitious variables. Since the only measurements were 


\section{CHAPTER VI. CONCLUSIONS AND FUTURE WORK}

the pressure drops, the measurement model contains only these pressure drops. Therefore, the matrix $H$ was introduced to fulfill these needs. This technique suggests that the system in a given operating region is a linear system rather than non-linear. The bed-height was calculated by the 2-region method, which used three pressure drops such as lean, dense, and total bed. This method used the proportional relation of the length and pressure drops.

With the standpipe dynamic and measurement models, the void fraction state was estimated by applying the sliding mode estimator technique, which is stable and robust. The bed-height was calculated from the void fractions since the bed-height separates the standpipe into two regions. The sliding mode estimator used the same concept as that of the sliding mode control strategy. The basic idea of the sliding mode control was that if there exists a positive energy function and its derivative is negative, then the controller guarantees stability and convergence. For an accurate estimation with the sliding mode technique, the solids circulation rate needs to be known. That information is available from the spiral installed in the standpipe of the CFCFB. In this research, however, the sliding mode estimator uses the estimated SCR rather than a measured SCR. Therefore, the sliding mode estimator requires only the pressure drop profiles in the standpipe and the aeration flow rates.

Finally, the control of the bed-height and SCR was simulated in this research. Since the entire CFCFB system model has not been developed, here the neural network was used to model the system model for the purpose of simulation. To reduce the training time in the neural network, the normalization of the inputs and outputs were applied, since the neurons of the neural network use the tansig functions. The controller was in a linear form because there are linear relationships between the control variable and responding variables (bed-height and SCR). The simulation results showed that the linear controller is capable of regulating both the bed-height and the SCR. 


\subsection{Future Work}

Throughout this research, the entire CFCFB system dynamic model was not available, but a one-dimensional mathematical model of the standpipe was. Since the SCR system model is in a linear form, this fact suggests that the entire CFCFB system model can be built in a linear form. It is possible to build these SCR linear system models in several operating regions, then combine SCR linear system models according to some criteria to be determined. In doing so, it is necessary to characterize the operating regions in accordance with CFCFB conditions.

In this research, all of the pressure taps in the standpipe are used to build the SCR system model and to estimate the state by the sliding mode observer. However, in industrial applications, the number of pressure taps should be as small as possible. In future work, the SCR system model and sliding mode estimator should be tried with less pressure profile information.

The control simulation results for the bed-height and the SCR presented in this research suggest the feasibility of experimental implementation in the standpipe of the CFCFB. 


\section{References}

[1] J. R. Grace, A. A. Avidan, and T. M. Knowlton. Circulating Fluidized Beds. Blackie Academic \& Professional, Chapman \& Hall, UK, first edition, 1997. 1.1

[2] P. Basu and S.A. Fraser. Circulating Fluidized Bed Boilers: Design and Operations. Butterworths, Bostong, 1991. 1.1

[3] D. King. Fluidized Catalytic Cracker: An Engineering Review. Ed. Potter and Nicklin, New York, 1992. 1.1

[4] A. Kullendorff and S. Andersson. A general review of combustion in circulating fluidized beds. In Circulating fluidized bed technology, pages 83 - 96. Pergamon Press, 1985. 1.1.1, 1.1.1, 1.1

[5] L. Wang, J. Fang, Z. Yang, H. Wang, G. Yan, H. Zai, H. Chen, and S. Liu. Performance of circulating fluidized bed boiler with compact external heat exchanger. In Proceedings of the International Conference on Energy Conversion and Application, pages 695 - 698, Wuhan, China, June 2001. Huanzhong University of Science and Technology. 1.1.1

[6] M. Hirsch, K. Janssen, and H. Serbent. The circulating fluidized bed as reactor for chemical and metallurgical processes. In Circulating fluidized bed technology, pages 329 - 339. Pergamon Press, 1985. 1.1.1, 1.2 


\section{REFERENCES}

[7] S. Kavidass, G.L. Anderson, and Jr G.S. Norton. Why build a circulating fluidized bed boiler to generate steam and eletric power. In Power-Gen Asia 2000, Bankok, Thailand, September 2000. 1.1.1

[8] A. M. Squires. The story of fluid catalytic craking: The first "circulating fluid bed". In Circulating fluidized bed technology, pages 1 - 19. Pergamon Press, 1985. 1.1.2

[9] SET Laboratories Inc. Catalytic cracking. Web:http://www.setlaboratories.com, May 17 2004. 1.1.2, 1.3, 1.1.2

[10] P. Koduru. Neural network modeling and control of cold flow circulating fluidized bed. Master's thesis, West Virginia University Institute of Technology, 2002. 1.2.1, $1.3,5.1$

[11] J. Park, , P. Famouri, A. A. Jalali, R. Turton, and E. Boyle. H-infinity based the state estimation on standpipe of cfcfb. In SMC 2003 Conference Proceedings, pages 2320 2325, Crystal City Hyatt Regency, Washington D.C. USA, October 2003. 1.2.1, 1.4, $1.3,2.2 .2,2.2 .2,3.1,3.6$

[12] J. Park, P. Famouri, Y. Huang, R. Turton, and E. J. Bolye. Bed height calculation and solid circulation rate estimation in standpipe of cfb. In CCCT 2003 Proceedings, pages 119 - 123, Oralndo, FL USA, July 2003. ISAS. 1.2.1, 1.4, 3.1, 3.6

[13] J. C. Ludlow, L. Lawson, L. Shadle, and M. Syamlal. Development of a spiral device for measuring the solids flow in a circulating fluidized bed. In International Conference on Circulating Fluidized Bed, pages 513 - 520, Niagara Falls, New York, 2002. 1.2.2, $1.2 .2,1.3,3.1$

[14] P. Stoholm, M. W. Fock, and U. Henriksen. Low temperature cfb gasifier conceptual ideas and applications. In Proceedings of the 15th International Conference on Fluidized Bed Combustion, Savannah, Goergia, May 1999. 1.3 


\section{REFERENCES}

[15] B. J. Milne, L. A. Behie, and F. Berruti. Recycling of waste plastics by ultrapyrolysis using an internally circulating fluidized bed reactor. Journal of Analytical and Applied Pyrolysis, 51(1 - 2):157 - 166, July 1999. 1.3

[16] R.E. Conn, K. Sellakumar, and A.E. Bland. Utilization of cfb fly ash for construction applications. In Proceedings of the 15th International Conference on Fluidized Bed Combustion, Savannah, Georgia, May 1999. 1.3

[17] C. Tame. Energy recovery from waste by use of fluidized bed technology. Int. Journal of Environmental Technology and Management, 1(1):192 - 201, 2001. 1.3

[18] L. Huilin, Y. Lidan, B. Rushan, and Z. Guangbo. Dynamic simulation of a circulating fluidized bed boiler of low circulating ratio with wide particle size distributions. In Proceedings of the 15th International Conference on Fluidized Bed Combustion, Savannah, Georgia, May 1999. 1.3

[19] A.D. Reichle. Fluid catalytic cracking hits 50 year mark on the run. Oil and Gas Journal, 90(20):41 - 48, May 1992. 1.3

[20] E. Herrmann and W. Weisweiler. Circulating fluidized bed reactor for catalytic gassolid reactions. International Chemical Engineering, 34(2):198 - 209, April 1994. 1.3

[21] S. Quyang and X. G. Li. Circulating fluidized bed as a catalytic reactor:experimental study. AIChE Journal, 41(6):1534 - 1542, June 1995. 1.3

[22] D. Beacon and R. Lundqvist. Advanced emission controls at mt. poso pyroflow circulating fluidized bed boiler. In Proceedings of the International Conference on Fluidized Bed Combustion, volume 1, pages 233 - 249, Montreal, Que., Can., April 1991. 1.3

[23] L. O. Lawson and L. J. Shadle. Characterization and indirect control of solids transfer 


\section{REFERENCES}

loops. In Poceedings of the 15th international conference on fluidized bed combustion, Savannah, GA, May 1999. 1.3

[24] L. J. Shadle, E. R. Monazam, and J. S. Mei. Circulating fluid bed operating regimes. In International Conference on Circulating Fluidized Bed, pages 255 - 262, Niagara Falls, New York, 2002. 1.3

[25] A. Davari, P. Koduru, and L. Shadle. Neural network control of cold flow circulating fluidized bed. In System Theory, 2003. Proceedings of the 35th Southeastern Symposium on, March 2003. 1.3

[26] H. Shim, P. Famouri, W. N. Sams, and E. J. Boyle. A state estimation of the standpipe of a circulating fluidized bed using an extended kalman filter. Proceedings of the 16th International Conference on Fluidized Bed Combustion, pages 130 - 140, May 2001. $1.3,1.5,2.1,2.2 .2,2.2 .2,2.3,3.1,3.6$

[27] J.J. Burkell, J.R. Grace, J. Zhao, and C.J. Lim. Measurement of solids circulation rates in fluidized beds. In Proceedings of Second International Conference on Circulating Fluidized Beds, pages 501 - 509, Pergamon, New York, 1988. 1.4, 3.1

[28] J. Lui and H. Bowen. Turbin meter for the measurement of bulk solids flow rate. Powder Technology, 82:145 - 151, 1995. 1.4, 3.1

[29] M. Moonen and J. Ramos. A subspace algorithm for balanced state space system identification. IEEE Trans. on Automatic Control, 38(11):1727-1729, November 1993. 1.4

[30] A. Swindlehurst, R. Roy, B. Ottersten, and T. Kailath. A subspace fitting method for identification of linear state-space models. IEEE Trans. on Automatic Control, 40(2): 311-316, February 1995. 1.4 


\section{REFERENCES}

[31] L. Ljung. System Identification: Theory for the user. Prentice Hall PTR, Upper Saddle River, NJ, 1999. 1.4, 1.5, 3.1, 3.2.2, 3.3.1, 3.3.1, 3.3.1, 3.3.2

[32] T. Brotherton and P. Caines. Arma system identification via the cholesky least squares method. IEEE Transactions on Automatic Control, 23(4):698 - 702, August 1978. 1.4, 3.1

[33] I. Goethals, T. Van Gestel, J. Suykens, and P. Van Dooren B. De Moor. Identification of positive real models in subspace identification by using regularization. IEEE Transactions on Automatic Control, 48(10):1843 - 1847, October 2003. 1.4

[34] E. Bai and K.M. Nagpal. Least squares type algorithms for identification in the presence of modeling uncertainty. IEEE Transactions on Automatic Control, 40(4): 756 - 761, April 1995. 1.4

[35] Z. Yan, C. Jin, and V. I. Utkin. Sensorless sliding-mode control of induction motors. IEEE Transactions on Industrial Electronics, 47(6):1286 - 1297, December 2000. 1.4, 4.1

[36] V. I. Utkin. Sliding mode control design priciples and applications to electic drives. IEEE Transactions on Industrial Electronics, 40(1):23 - 36, February 1993. 1.4, 4.1

[37] A. Benchaib, A. Rachid, E. Audrezer, and M. Tadjine. Real-time sliding-mode observer and control of an induction motor. IEEE Transactions on Industrial Electronics, 46(1):128 - 137, February 1999. 1.4, 4.1

[38] A. Derdiyok, H. Rehman, M. K. Guven, N. Inanc, and L. Xu. A robust slidng mode observer for speed estimation of induction machine. In Applied Power Electronics Conference and Exposition, Sixteenth Annual IEEE. APEC 2001, 2001. 1.4, 4.1 


\section{REFERENCES}

[39] H. Rehman, A. Derdiyok, M.K. Guven, and X. Longya. A new current model flux observer for wide speed range sensorless control of an induction machine. IEEE Transactions on Power Electronics, 17(6):1041 - 1048, November 2002. 1.4

[40] M. Rodic and K. Jezernik. Speed-sensorless sliding-mode torque control of an induction motor. IEEE Transactions on Industrial Electronics, 49(1):87 - 95, February 2002. 1.4

[41] H.D. Pantino and D. Liu. Neural network-based model reference adaptive control system. IEEE Transactions on Systems, Man, and Cybernatics, 30(1):198 - 204, February 2000. 1.4, 5.3

[42] J. Chen and Y. Xi. Nonlinear system modeling by competitive learning and adaptive fuzzy inference system. IEEE Transactions on Systems, Man and Cybernatics, 28(2): 231 - 238, May 1998. 1.4, 5.3

[43] R.G. Hutchins. Neural network topologies and training algorithms in nonlinear system identification. In IEEE International Conference on Systems, Man and Cybernatics, volume 3, pages 2512 - 2515. IEEE Systems, Man and Cybernatics, October 1995. $1.4,5.3$

[44] P.J. Antsaklis. Neural networks for control systems. IEEE Transactions on Neural Networks, 1(2):242 - 244, June 1990. 1.4, 5.3

[45] A.J. Owens. Process modeling and control using a single neural network. In IEEE International Conference on Systems, Man and Cybernatics, volume 2, pages 1475 1480. IEEE Systems, Man and Cybernatics, October 1995. 1.4, 5.3

[46] B. Fernandez, A.G. Parlos, and W.K. Tasi. Nolinear dynamic system identification using artificial neural networks. In IJCNN International Joint Conference, volume 2, pages 133 - 141. IEEE Neural Networks, June 1990. 1.4, 5.3 


\section{REFERENCES}

[47] M.T. Hagan and M.B. Menhaj. Training feedforward networks with the marquardt algorithm. IEEE Transactions, 5(6):989 - 993, November 1994. 1.4, 5.3.1

[48] J. Park, L. Wang, L. A. Hornak, and P. Famouri. Microstructure torque estimation using mems optical monitoring. In Sensors, 2002. Proceedings of IEEE, volume 2, pages $1746-1750,2002$. 1.5, 4.1, 4.1

[49] J. F. Richardson and W. N. Zaki. Sedimentation and fluidisation. Transactions of Institute of Chemical Engineering, 32:35, 1954. 2.1, 2.2.2

[50] J. M. Coulson, J. F. Richardson, J. R. Backhurst, and J. H. Harker. Chemical Engineering. Butterworth-Heinemann, 225 Wildwood, Woburn MA USA, 1998. 2.1, 2.3

[51] R. C. Dorf and R. H. Bishop. Modern control systems. Addson-Wesley Publishing Company, 7th edition, 1995. 2.2.2

[52] N. Amjady. Short-term hourly load forecasting using time-series modeling with peak load estimation capability. IEEE Transactions on Power Systems, 16(3):498-505, August 2001. 3.2

[53] T. W Chow and H. Z. Tan. Hos-based nonparametric and parametric methodologies for machine fault detection. IEEE Transactions on Industrial Electronics, 47(3):10511059, October 2000. 3.2

[54] U. Forssell and L. Ljung. Identification of unstable systems using output error and box-jenkins model structures. IEEE Transactions on Automatic Control, 45(1):137141, January 2000. 3.2

[55] G. E. P. Box and G. M. Jenkins. Time Series Analysis forecasting and control. HoldenDay, Oakland, California, 1976. 3.2.1 


\section{REFERENCES}

[56] R. H. Shumway and D. S. Stoffer. Time Series Analysis and Its Applications. SpringerVerlag, New York, NY, 2000. 3.2.1

[57] S. Haykin. Adaptive Filter Theory. Prentice Hall, Inc, Upper Saddle River, NJ, 1996. 3.2 .2

[58] A. Gelb. Applied Optimal Estimation. The M.I.T. Press, Cambridge, MA, 2001. 3.4, 3.4.1, 3.4.1

[59] Y. Xiong and M. Saif. Sliding mode observer for nonlinear uncertain systems. IEEE Transactions on Automatic Control, 46(12):2012 - 2017, December 2001. 4.1

[60] M. S. Islam, I. Husain, R. J. Veillette, and C. Batur. Design and performance analysis of sliding-mode observers for sensorless operation of switched reluctance motors. IEEE Transaction on Control Systems Technology, 11(3):383 - 389, May 2003. 4.1

[61] J. Jacque, E. Slotine, and W. Li. Applied Nonlinear Control. Printice-Hall Inc., Englewood Cliffs, New Jersey, second edition, 1991. 4.1, 4.2.1, 4.2.2

[62] N. S. Nise. Control Systems Engieering. The Benjamin/Cummings Publishing Company, 390 Bridge Parkway, Redwood City, CA 94065, second edition, 1995. 4.2.1

[63] M.T. Hagan, H. Demuth, and M. Beale. Neural Network Design. Boulder Co., Boston, MA, 1996. 5.3.1, 5.3.1

[64] H. B. Demuth. Neural network toolbox for use with MATLAB: user's guide. Mathworks, Natick, MA, 1998. 5.3.1 